University of Mannheim / Department of Economics

Working Paper Series

Segmentation versus Agglomeration: Competition between Platforms with Competitive Sellers

\author{
Heiko Karle Martin Peitz Markus Reisinger
}

Working Paper 17-03

November 2017 


\title{
Segmentation versus Agglomeration: Competition between Platforms with Competitive Sellers*
}

\author{
Heiko Karle $\quad$ Martin Peitz Markus Reisinger $^{\ddagger}$
}

November 9, 2017

\begin{abstract}
For many products, platforms enable sellers to transact with buyers. We show that the competitive conditions among sellers shape the market structure in platform industries. If product market competition is tough, sellers avoid competitors by joining different platforms. This allows platforms to sustain high fees and explains why, for example, in some online markets, several homogeneous platforms segment the market. Instead, if product market competition is soft, agglomeration on a single platform emerges, and platforms fight for the dominant position. These insights give rise to novel predictions. For instance, market concentration and fees are negatively correlated in platform industries, which inverts the standard logic of competition.
\end{abstract}

Keywords: intermediation, two-sided markets, market structure, price competition, endogenous segmentation

JEL-classification: L13, D43.

${ }^{*}$ We are grateful to Mark Armstrong, Özlem Bedre-Defolie, Paul Belleflamme, Luis Cabral, Emilio Calvano, Jay Pil Choi, Alex de Cornière, Reyer Gerlagh, Michael Grubb, Hanna Halaburda, Maarten Janssen, Johannes Johnen, Bruno Jullien, Damian Kozbur, Yassine Lefouili, Marc Möller, Nick Netzer, Georg Nöldeke, Amedeo Piolatto, Sven Rady, Pierre Regibeau, Armin Schmutzler, Tim Simcoe, Timothy Wyndham, Julian Wright, Ali Yurukoglu, and participants at various conferences and seminars. Martin Peitz gratefully acknowledges support by the Deutsche Forschungsgemeinschaft (PE 813/2-2).

${ }^{\dagger}$ Department of Economics, Frankfurt School of Finance \& Management, Adickesallee 32-34, 60322 Frankfurt am Main, Germany; E-Mail: h.karle@fs.de.

${ }^{\ddagger}$ Department of Economics and MaCCI, University of Mannheim, 68131 Mannheim, Germany; EMail: Martin.Peitz@gmail.com; also affiliated with CEPR, CESifo, and ZEW.

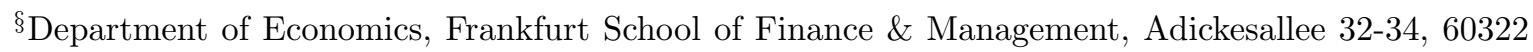
Frankfurt am Main, Germany. E-Mail: m.reisinger@fs.de; also affiliated with CESifo. 


\section{Introduction}

In many industries, platforms offer intermediation services and play the essential role of enabling transactions between buyers and sellers - more prominently so, with the migration of trade from physical venues to the Internet. For example, in the rental market, the main bulk of matching landlords and tenants is done via Internet platforms such as Rightmove and Zoopla in the UK, or Immobilienscout24 and Immowelt in Germany. Other examples include the used car market and markets for other used items or collectibles, in which a large fraction of transactions is initiated via portals.

The market structure for intermediation services differs considerably across industries and space. While, for example, Ebay dominates the second-hand market in many product classes in several countries, the rental housing market is often segmented, and two (or more) platforms have non-negligible market shares 1

In this paper, we provide a theoretical framework to examine these differences in platform market structure. We find that the market structure is shaped by the competitive conditions in the product market. These conditions are responsible for the fees set by platforms and, thus, for platforms' profits and for the number of active platforms.

As is well-known, platform markets may have the tendency to tip due to positive cross-group external effects between buyers and sellers (i.e., each buyer benefits from more sellers on the same portal and vice versa). This has been shown in the seminal work by Caillaud and Jullien $(2001,2003)$ and rationalizes the phenomenon of market agglomeration, in which all agents locate on a single platform. However, in several industries, two or more platforms have non-negligible market shares, and agents join different platforms. The existing literature explains market segmentation with platforms offering differentiated matching services (e.g., Rochet and Tirole, 2003; Armstrong, 2006).

In the above examples, and more broadly for many Internet platforms, there is little room for service differentiation; that is, platforms offer services that often appear to be quite the same. Therefore, it remains a puzzle how competing platforms share the market and earn positive profits.

Our answer to this puzzle is that multiple homogeneous platforms can serve the role of relaxing competition between sellers in the product market. In a nutshell, if sellers decide to be active on different platforms, some buyers will not be informed about all offers, which, in turn, relaxes competition between sellers. Platforms benefit from this provision of endogenous segmentation by charging sellers larger fees. Thus, multiple

\footnotetext{
${ }^{1}$ Even within the same industry, differences between countries can be observed. For example, in the daily deals market, in which platforms mediate transactions of discounted products and services through coupons, Groupon has a dominant position with market shares of around $80 \%$ in several European countries, whereas in the US the market is more segmented, with Groupon and LivingSocial being the market leaders (Kim, Lee and Park, 2017).
} 
homogeneous platforms earn positive profits.

We identify the competitive conditions in the product market as the key driver of the arising market structure. If product market competition is soft (e.g., because sellers offer highly differentiated products), agglomeration forces dominate. Then, platforms follow a strategy of "play hard and fight it out" to become the dominant platform, which leads to low fees (at least in the short term, when the number of platforms is exogenous). If, instead, product market competition is tough, multiple platforms segment the market to relax seller competition. Platforms then "play soft" and charge high fees.

Dudey (1990) and Ellison and Fudenberg (2003) demonstrate that under tough product market competition, sellers benefit from allocating at different marketplaces. In those papers, however, marketplaces are not managed by platforms and do not charge fees to sellers. Our analysis advances this literature by confirming that market segmentation can arise even with fee-setting platforms. Yet, we find that fees are strictly positive under market segmentation.

Overall, our paper provides testable predictions of how the competitive environment faced by sellers drives the equilibrium market structure and the platforms' equilibrium choice of listing fees. Tough competition between sellers implies high platform fees and profits. Therefore, the correlation between competition in the product market and competition in the market for intermediation services is negative. In addition, a lower market concentration in platform markets due to multiple active platforms go together with higher listing fees. This implies that the relation between the Hirshman-Herfindahl Index (HHI) and the markup is reversed in platform markets versus standard oligopoly markets.

To provide casual evidence for the existence of market segmentation in platform industries, we carried out searches on the German rental platforms Immobilienscout24 and Immowelt. An example is the search for rental apartments in Frankfurt am Main, Germany with the following search criteria: "at least 3 rooms"; "at least $100 \mathrm{~m}^{2}$ "; and "distance less than 1 kilometer to the centre." The search on November 23, 2015 resulted in 12 matches on each portal. We report the matches in Table 1 in ascending order of rental price by stating the square meters of the apartment and the rental price in Euros. Out of these 12 matches, only two could be found on both platforms 2 This appears to be consistent with the idea of market segmentation by platforms.

Our theoretical framework applies also to industries beyond e-commerce. A case in point are industry standards; for example, the modem standard for end-user Internet access in the 1990s: Augereau, Greenstein, and Rysman (2006) find that two different, but functionally equivalent modem standards were used by Internet Service Providers (ISPs, which would be the sellers in our model) despite positive effects of standardization.

\footnotetext{
${ }^{2}$ Offer 11 on Immobilienscout24 is the same apartment as offer 9 on Immowelt, and offer 12 on Immobilienscout24 is the same apartment as offer 11 on Immowelt.
} 


\begin{tabular}{|c|cc|cc|}
\hline & \multicolumn{2}{|c|}{ Immobilienscout24 } & \multicolumn{2}{c|}{ Immowelt } \\
\hline 1. & $m^{2}: 103.78 ;$ & Rent:1.350 & $m^{2}: 111.00 ;$ & Rent:1.285 \\
\hline 2. & $m^{2}: 110.00 ;$ & Rent:1.450 & $m^{2}: 104.00 ;$ & Rent:1.290 \\
\hline 3. & $m^{2}: 100.00 ;$ & Rent: 1.450 & $m^{2}: 117.00 ;$ & Rent:1.350 \\
\hline 4. & $m^{2}: 105.90 ;$ & Rent: 1.450 & $m^{2}: 103.56 ;$ & Rent:1.490 \\
\hline 5. & $m^{2}: 129.02 ;$ & Rent:1.548 & $m^{2}: 114.00 ;$ & Rent:1.550 \\
\hline 6. & $m^{2}: 124.74 ;$ & Rent:1.597 & $m^{2}: 145.00 ;$ & Rent:1.650 \\
\hline 7. & $m^{2}: 142.00 ;$ & Rent:1.700 & $m^{2}: 100.00 ;$ & Rent:1.800 \\
\hline 8. & $m^{2}: 136.00 ;$ & Rent:1.890 & $m^{2}: 140.00 ;$ & Rent:1.970 \\
\hline 9. & $m^{2}: 137.48 ;$ & Rent:2.007 & $m^{2}: 140.00 ;$ & Rent:2.450 \\
\hline 10. & $m^{2}: 173.00 ;$ & Rent:2.290 & $m^{2}: 160.00 ;$ & Rent:2.800 \\
\hline 11. & $m^{2}: 140.00 ;$ & Rent:2.450 & $m^{2}: 152.00 ;$ & Rent:2.830 \\
\hline 12. & $m^{2}: 152.00 ;$ & Rent:2.830 & $m^{2}: 200.00 ;$ & Rent:3.200 \\
\hline
\end{tabular}

Table 1: Apartment offers in ascending order of the rental price

This helped ISPs to reduce competition (by creating switching costs for consumers). As a result, the two modem standards obtained similar market shares and, thus, segmented the market.

In our baseline model, multiple platforms compete on listing fees charged to sellers. Buyers prefer platforms with many sellers, and vice versa. Sellers offer a single product that belongs to one out of many different product categories, and there are multiple sellers within the product category competing with each other. To present the results in the simplest way, we focus on the case with only two platforms and two sellers per category. All of our results extend to a general number of platforms and sellers and to per-transaction fees or two-part tariffs. After platforms set their listing fees, sellers and buyers decide simultaneously which platform to join and, thus, play a coordination game. We show that the selection criterion of coalition-proofness, in combination with profit dominance of sellers, generates a clear-cut equilibrium prediction in this coordination game. This allows us to establish necessary and sufficient conditions when either agglomeration or segmentation emerges.

A tipping equilibrium prevails if the degree of competition between sellers is low. This is in line with the case of Ebay in the example above, as competition between sellers of a particular second-hand product on Ebay is presumably relatively soft. Buyers are then informed about all offers, implying that sellers are in competition with each other. However, demand is also higher as all buyers are on the same platform. The effect of increased demand dominates increased competition. Platforms compete fiercely to win the market, which leads to a Bertrand-style competition between platforms, and their listing fees are driven down to marginal cost $t^{3}$

\footnotetext{
${ }^{3}$ Agglomeration, therefore, does not imply that a platform acts as a monopolist. Instead, another
} 
By contrast, if competition between sellers in a product category is sufficiently intense, they prefer to be active on different platforms. Buyers will split on the two platforms and do not become informed about all offers. Thus, platforms segment the market, and competition between sellers is relaxed. This finding is in line with the examples of Rightmove vs. Zoopla and Immobilienscout24 vs. Immowelt on the rental market, as landlords and rental agencies often compete for the best tenants. Segmentation then allows platforms to obtain strictly positive profits. If a platform were to deviate from the associated equilibrium listing fees by charging a lower fee, sellers would not have an incentive to switch to this platform, as this would intensify competition among them.

If the degree of competition between sellers is moderate, a mixed-strategy equilibrium in listing fees occurs, in which platforms segment the market with positive probability. Confirming the result described above, if the degree of competition between sellers gets larger, the probability for segmentation increases, and so does the expected profit of platforms, as they charge higher fees.

While our baseline model features single-homing of agents on both sides, we subsequently allow for multi-homing buyers and sellers and show that our solution to the puzzle that multiple platforms share the market carries over. We find that platforms obtain lower profits with seller multi-homing as multi-homing makes agglomeration more likely.

Existing literature with differentiated platforms has shown that seller multi-homing allows competing platforms to exert monopoly power over sellers and to possibly increase their profits. By contrast, we find that seller multi-homing may affect the market structure and has, thereby, a different effect on platform profits: Due to multi-homing, sellers may profitably deviate from segmentation by becoming active on both platforms and, thus, making offers to all buyers. This might render segmentation unstable. Then, agglomeration occurs, and platforms unambiguously receive lower profits.

From a welfare perspective, segmentation is inefficient. The reason is that matching quality is lower, as buyers are not informed about all offers, and the deadweight loss is higher than under agglomeration due to higher product market prices. As a consequence for competition policy, restraints such as exclusive dealing contracts, which platforms may impose on sellers, are anticompetitive, as they prevent seller multi-homing and, thus, are likely to induce segmentation.

In the remainder of this section, we discuss the related literature. In Section 2, we set out the baseline model and, in Section 3, characterize the equilibrium. In Section 4, we analyze the effects of multi-homing by buyers and sellers. We present generalizations of our baseline model, including the use of alternative platform pricing instruments, any 
number of platforms and sellers, and a different equilibrium selection in Section 5. In Section 6, we discuss policy implications and empirical predictions. Section 7 concludes. All proofs are relegated to the Appendix.

Related Literature Our paper contributes to the literature on competition in two-sided markets, pioneered by Caillaud and Jullien (2001, 2003), Rochet and Tirole (2003, 2006), and Armstrong (2006). Caillaud and Jullien (2001, 2003) analyze homogeneous platforms and show that the market tips to one platform under relatively general conditions. Rochet and Tirole (2003, 2006) and Armstrong (2006), by contrast, consider platforms that are sufficiently differentiated so that no tipping occurs. The focus of these papers (and generally in the two-sided market literature) is on cross-group externalities between agents of both sides but not on competition between agents on the same side (as sellers do in our model). Armstrong (2006) considers seller competition in an extension, and shows that platforms may restrict seller competition to obtain higher profits. In contrast to our paper, in his framework, all platforms are active due to exogenous differentiation.

A few papers in the two-sided markets literature analyze competition between sellers. Nocke, Peitz and Stahl (2007), Galeotti and Moraga-González (2009), and Gomes (2014) analyze platform ownership, search, and optimal auction design, respectively, but consider a monopoly platform, whereas Belleflamme and Toulemonde (2009) study competition between a for-profit and a not-for-profit platform. Dukes and Gal-Or (2003) and Hagiu (2006) consider competition between for-profit platforms and analyze either exclusivity contracts or price commitment by platforms 4 None of these papers analyzes how the market structure depends on seller competition 5

Ellison, Fudenberg, and Möbius (2004) consider competition between two auction sides. They derive conditions for sellers to be active on different platforms, as this lowers the seller-buyer ratio on each platform and leads to higher prices. Ellison and Fudenberg (2003) provide general conditions such that tipping does not occur in markets with crossgroup external effects. The key difference from our paper is that they do not consider fee-setting by platforms (i.e., fees are zero in their setup) 6

The literature on firms' location decisions analyzes the benefits and costs of clustering from a different angle. For example, Dudey (1990) shows that sellers prefer agglomeration in one marketplace over fragmentation, as lower product prices are more than offset by

\footnotetext{
${ }^{4}$ In line with the previous literature, Hagiu (2006) shows that if commitment is not possible and agents single-home, an agglomeration equilibrium with zero profits emerges.

${ }^{5}$ An exception is Halaburda, Piskorski and Yilidrim (2017) who consider a matching market with heterogeneous agents. They show that agents with low outside options prefer a platform with restricted choice which leads to market segmentation with platforms of different size. By contrast, in our paper segmentation occurs with homogeneous agents on either side and leads to symmetric platforms.

${ }^{6}$ Ellison, Fudenberg, and Möbius (2004), in their Section 7, briefly analyze platform pricing. However, since they do not make assumptions on equilibrium selection in the coordination game between sellers and buyers, they do not provide a unique mapping from fees to market structure.
} 
increased demand. Stahl (1982) demonstrates that a similar effect arises if buyers are attracted by a greater variety of goods. Church and Gandal (1992) analyze a related model applied to the software market. In contrast to our paper, marketplaces are open platforms in the sense that access is free. Instead, we are interested in markets with fee-setting platforms and the resulting market structure.7

Our paper also contributes to the literature on price comparison websites. In the seminal paper, Baye and Morgan (2001) show how homogeneous firms obtain positive profits, even if a website informs buyers about all prices. The idea is that firms still sell in their local market, where buyers are not informed about all prices. This leads to price dispersion in equilibrium. This result has been tested empirically (e.g., Brown and Goolsbee, 2002; Baye and Morgan, 2004) and the theoretical framework has been extended (e.g., Ronayne, 2015). In contrast to these papers, we analyze competition between websites in addition to competition between sellers.

\section{The Setup}

\subsection{Baseline Model}

We consider markets in which buyers and sellers trade via platforms. In what follows, we describe the three types of agents-platforms, sellers, and buyers.

Platforms. Two homogeneous platforms $A$ and $B$ offer listing services to sellers. The platforms enable transactions between sellers of products or services and their prospective buyers. To be listed on platform $i$, a seller has to pay a listing fee $f_{i}, i \in\{A, B\}$. Such listing fees are prevalent in markets in which platforms cannot or do not monitor the sale of a product - for example, in the housing or rental market. Buyers can access platforms for free 8 For simplicity, we assume that all platform costs are zero.

Sellers. Sellers have to decide which, if any, platform to join. In the baseline model, they cannot be active on both platforms (i.e., sellers single-home) - in Section 4.2, we show that our results carry over to the case with multi-homing sellers 9 The product of each seller belongs to a product category. There is a mass 1 of such categories, indexed by $k \in[0,1]$.

\footnotetext{
${ }^{7}$ An exception is Gehrig (1998), who considers Hotelling competition between marketplaces and competition on the circle (Salop, 1979) between sellers. He shows that agglomeration equilibria may emerge (with positive platform profit), despite platform differentiation.

${ }^{8}$ We discuss the case of two-sided pricing in Section 5.4 .

${ }^{9}$ In some industries, single-homing is a natural assumption. For example, in the market for private accommodations, apartment owners have difficulties synchronizing the calendars when they are active on more than one platform. This favors single-homing. Other examples are the daily deals market, in which sellers cannot offer the same deal on more than one platform, or the modem market for Internet access in which each ISP can use only one modem for technological reasons.
} 
For simplicity, we assume that there are two sellers in each product category. ${ }^{10}$ Sellers are symmetric and obtain an equilibrium per-buyer profit $\pi^{d}$ in duopoly. If buyers can buy from only one of the sellers because only this seller is listed on the platform that buyers are patronizing, the seller makes a monopoly profit $\pi^{m}$ per buyer, with $\pi^{m} \geq \pi^{d}$. Our formulation implies that per-buyer profit in duopoly and monopoly is independent of the number of buyers. At the end of this section, we provide several micro-foundations that fulfill this property. However, we also demonstrate that our qualitative results hold more generally. For the sake of concreteness, we assume that sellers engage in price competition and set uniform prices to buyers. We denote the symmetric equilibrium duopoly price by $p^{d}$ and the monopoly price by $p^{m}$.

In the baseline model, there is no interdependence between categories 11 This represents the fact that, although platforms usually list many items (a continuum in our model), there is competition between only a few of them. For example, a price comparison website often has thousands or even millions of listed products, but only a few items match a buyer's search and are displayed to the buyer. Similarly, housing platforms are host to many houses and apartments, but a buyer seeking a house of a particular size in her preferred city is not interested in listings in other categories.

Buyers. Each buyer single-homes - that is, she decides to be active on (up to) one platform. 12 She is interested in a single product category and derives a positive gross utility only from products in this category - see, e.g., Burguet, Caminal, and Ellman (2016) for a similar structure. There is mass 1 of buyers per product category. When visiting a platform, a buyer becomes informed about her preferred product category and the price of all products listed on the platform. ${ }^{13}$ If a platform lists sellers' products from a fraction $\alpha \in[0,1]$ of all categories, a buyer expects to find a product from her preferred category with probability $\alpha$.

A buyer obtains a different (indirect) utility if one or two sellers are listed in her preferred category. Prior to observing the idiosyncratic taste realization within this category, the buyer obtains an expected utility of $V^{d}$ if she expects two sellers to be listed in her preferred category. If she expects only one seller to be listed, her expected utility is $V^{m}<V^{d}$. The reason for this inequality is twofold: First, if two sellers are listed, they charge the duopoly price $p^{d}$, which, in many instances, is less than $p^{m}$. Second, if

\footnotetext{
${ }^{10}$ In Section 5.2 we show that all our results carry over to the situation with a general number of sellers per category and a general number of platforms.

${ }^{11}$ We discuss several possible interactions between categories in Section 5.5

${ }^{12}$ In Section 4.1, we provide the analysis with multi-homing buyers and demonstrate that our main insights remain valid.

${ }^{13}$ The assumption that a buyer learns her preferred category only after deciding which platform to visit is only made to simplify the analysis. All results would also hold if buyers knew their preferred category already at the outset.
} 
sellers are differentiated, and buyers have a taste for variety, a buyer will find a product closer to her preferences or may buy a positive amount of both products if two sellers are listed instead of only one.

Timing. The timing is as follows:

1. Platforms $A$ and $B$ set listing fees $f_{A}$ and $f_{B}$, respectively.

2. Sellers and buyers make a discrete choice between platforms $A$ and $B$, and the outside option (normalized to zero).

3. Sellers in each category set product prices.

4. Buyers observe all offers on the platform they are visiting and make their purchasing decisions.

We make three observations regarding our setup. First, according to our timing, sellers decide where to list before setting their prices on the product market. This is the relevant timing in most applications because the choice of platform is typically longerterm than the pricing decision. Therefore, sellers set prices after learning about the number of competitors in the product market. In addition, listing fees are often paid on a subscription basis, which makes them lumpy. By contrast, prices charged by the sellers are flexible.

Second, listing fees do not enter the pricing decisions of sellers in the third stage because they are "fixed" costs for sellers (which are, in addition, sunk when sellers set prices). As we show in Section 5.1, our results still hold with per-transaction fees or revenue shares, which affect sellers' pricing decisions.

Third, we do not impose a particular model of buyer-seller interaction for the subgame starting at stage 3 and, instead, use a reduced-form approach with several microfoundations provided in Section 2.2 .

Payoffs. The profit of platform $i$ is the number of sellers active on platform $i$ multiplied by the listing fee $f_{i}$. The profit of a seller who is listed on platform $i$ is $\beta_{i} \pi-f_{i}$, where $\beta_{i}$ is the fraction of buyers in the seller's category that are active on platform $i$, and $\pi$ is either $\pi^{m}$ if the rival seller is not listed on platform $i$ or $\pi^{d}$ if the rival also lists on platform $i$. As mentioned above, the utility of a buyer is $V^{d}$ or $V^{m}$ and, thus, depends on the number of sellers listed in the buyer's preferred category; the utility is 0 if none of those sellers is listed on the platform.

Solution Concept. Our solution concept is subgame perfect Nash equilibrium. We assume the following tie-breaking rule. If buyers expect one seller in each category to list on platform $A$ and the other seller on platform $B$, half of the buyers in each category join platform $A$ and the other half platform $B$. A natural interpretation is that each buyer mixes with equal probability to be active on either platform $A$ or $B$. Since there is a continuum of buyers, both platforms will, in fact, be patronized by one half of the 
buyers ${ }^{14}$ As we point out below, this assumption is not crucial for the results and can be relaxed, allowing for unequal distributions of buyers in the case of indifference.

In the second stage, buyers and sellers face a coordination game on which platform(s) to be active, which may lead to a multiplicity of equilibria. To deal with this well-known issue in two-sided markets, we impose the refinement of coalition-proofness (see e.g., Bernheim, Peleg, and Whinston, 1987a, 1987b). That is, we select only Nash equilibria that are stable against deviations by coalitions of sellers and buyers; and, within the coalition, no subset of sellers and buyers benefits from a further deviation 15 In addition, when coalition-proofness is not sufficient to obtain equilibrium uniqueness, we select equilibria that are profit-dominant for sellers. We will show that the joint application of these refinements leads to a unique equilibrium outcome at stage 116

A justification of the refinement is that the outcome is equivalent to the outcome of a sequential game in which sellers decide which platform to join before buyers do, as considered, for example, by Hagiu (2006), and sellers play a coalition-proof Nash equilibrium. In Section 5.3, we analyze the mirror case, in which the payoff-dominant equilibrium for buyers is selected, and we demonstrate that the main insights of our analysis will be unchanged.

Summary statistic. As will become clear in Section 3, the key summary statistic for our equilibrium characterization is the ratio $\pi^{d} / \pi^{m}$, which is an inverse measure of the degree of product market competition and takes values in $[0,1]$. It is determined by the buyer-seller interaction at stages 3 and 4 .

\subsection{Micro-Foundation of the Buyer-Seller Interaction}

Buyers' choices in stage 4 and sellers' pricing decisions in stage 3 are straightforward: In the fourth stage, a buyer buys one or both products in her preferred product category according to her demand function, provided that there is at least one listed seller on the platform where the buyer is active. In the third stage, sellers set $p^{d}$ in case they face a

\footnotetext{
${ }^{14}$ Another interpretation is that platforms are differentiated by different platform designs but that this differentiation is negligibly small. For example, platforms are differentiated along a Hotelling line, and the transport cost parameter $t$ goes to zero. This means that buyers ex ante have lexicographic preferences, in the sense that they prefer the platform with a larger number of sellers. Buyers decide according to their preference for different platform designs only if they expect this number to be the same across platforms.

${ }^{15}$ In our game, a coalition-proof Nash equilibrium is equivalent to a Strong Nash equilibrium (Aumann, 1959), which ignores deviations by subcoalitions. This is due to buyers within each category being symmetric and sellers benefiting from the presence of more buyers.

${ }^{16}$ Our equilibrium concept differs from the one imposed by Caillaud and Jullien (2003) and Jullien (2011), who consider favorable expectations for one platform (the incumbent) in case of a fee deviation by the rival platform (the entrant). By contrast, in our model, agents form expectations after observing platform prices, and expectations are symmetric.
} 
competitor in their product category on the platform and $p^{m}$ in case of monopoly.

In this subsection, we provide several micro-foundations of the buyer-seller interaction at stages 3 and 4 and, thus, determine the equilibrium per-buyer profits $\pi^{d}$ and $\pi^{m}$. The first two examples are widely-used oligopoly models: a discrete product choice model (Hotelling) and a representative consumer model (Bowley, 1924, or Singh and Vives, 1984). Both examples fulfill all our assumptions, and we use the example on Hotelling competition as our lead example ${ }^{17}$ The third example extends price competition to a simple setting of thin markets in which there is only a small number of buyers and capacity-constrained sellers (as in the housing market). In the fourth example, sellers do not use prices as their strategic variable but quantities. The latter two examples do not fulfill all assumptions set out above (as there is only a finite number of buyers in Example 3, and sellers compete in quantities in Example 4). However, because only $\pi^{d}$ and $\pi^{m}$ are relevant for our results, we can restate the model so that it is in line with Example 3 or 418

\section{Example 1: Price competition in the Hotelling model.}

Consider Hotelling competition in each product category. Each seller is located at one of the extreme points of the unit interval in a particular category-i.e., a seller $j$ is characterized by its category $k_{j}$ and its location $l_{j}$ on the unit interval, $\left(k_{j}, l_{j}\right) \in[0,1] \times$ $\{0,1\}$. The buyers' valuation of a product at the ideal location in the preferred category equals $v$. If a buyer likes category $k$ and is located at $x_{k}\left(\right.$ with $\left.\left(k, x_{k}\right) \in[0,1] \times[0,1]\right)$, her utility from buying one unit of seller $j$ 's product in this product category is $v-t\left|x_{k}-l_{j}\right|-p_{l_{j}}$ where $t>0$ captures the degree of product differentiation. Her utility is zero for products in all categories that are not equal to $k$. Price competition among Hotelling duopolists leads to equilibrium prices $c+t$ and equilibrium profits $\pi^{d}=t / 2$ per unit mass of buyers. ${ }^{19}$ A monopoly seller sets price $p^{m}=(v+c) / 2$, and its profit is $\pi^{m}=(v-c)^{2} /(4 t)$ per unit mass of buyers if the market is not fully covered. This is the case if $t \geq(v-c) / 2$. In this parameter range, $p^{m} \leq p^{d}$. For $t<(v-c) / 2$, there is full coverage, and the monopolist sets $p^{m}=v-t$. Its profit is $\pi^{m}=v-t-c$.

In the Hotelling model, the ratio $\pi^{d} / \pi^{m}$ is given by $\sqrt{2} t /(v-c)$ if $(v-c) / 2 \leq t \leq$ $2(v-c) / 3$ and by $t /[2(v-t-c)]$ if $t<(v-c) / 2$. It follows that $\pi^{d} / \pi^{m} \geq 1 / 2$ for $t \geq(v-c) / 2$, and vice versa. That is, if products are sufficiently differentiated, twice the duopoly profit is larger than the monopoly profit.

Example 2: Price competition with a representative consumer with linear demand and

\footnotetext{
${ }^{17}$ Other representative consumer models - for instance, with CES or logit demand-would work as well.

${ }^{18}$ Another micro-foundation that we do not develop here but that also fits our assumptions are models of sequential product search (e.g., Wolinsky, 1986, and Anderson and Renault, 1999).

${ }^{19}$ The upper bound on $t$ is $2(v-c) / 3$, as the buyer who is indifferent between both sellers would not obtain a positive utility otherwise.
} 
differentiated products.

Suppose that buyers with the same preferred category have an indirect utility function of $q_{1}+q_{2}-1 / 2\left(q_{1}^{2}+q_{2}^{2}\right)-\gamma q_{1} q_{2}-p_{1} q_{1}-p_{2} q_{2}$, with $\gamma \in[0,1]$ expressing the degree of substitutability between products. This is a representative consumer setting in which each buyer obtains utility from positive quantities of each product in her preferred category. Maximizing this utility function with respect to $q_{1}$ and $q_{2}$, we obtain the indirect demand functions $p_{i}=1-q_{i}-\gamma q_{-i}, i=1,2$. Inverting this demand system yields the direct demand functions $q_{i}=\left(\beta-\gamma-p_{i}+\gamma p_{-i}\right) /\left(1-\gamma^{2}\right)$, for $i=1,2$.

Duopoly equilibrium profit per buyer is $\pi^{d}=\left[(1-\gamma)(1-c)^{2}\right] /\left[(1+\gamma)(2-\gamma)^{2}\right]$. For a monopolist, the direct demand is $q_{i}=1-p_{i}$ and the per-buyer profit is $\pi^{m}=(1-c)^{2} / 4$. Thus, the ratio $\pi^{d} / \pi^{m}$ is given by

$$
\frac{4(1-\gamma)}{(1+\gamma)(2-\gamma)^{2}}
$$

which is above $1 / 2$ if $\gamma$ is lower than approximately 0.62 .

Example 3: Thin markets.

In this example, we consider capacity-constrained sellers that each can offer only one unit of a product - the analysis can be extended to allow for sellers with a finite number of products to sell. Real-world applications include the housing and rental market, and the market for collectibles in which owners often have only one unit to sell.

Suppose that, in each category, there are two sellers and finitely many buyers $M_{B}>1$. The example differs from the baseline model, as there is no continuum of buyers. To keep the exposition simple, suppose that there are two buyer types with valuation $R \in\{\underline{R}, \bar{R}\}$, with $0 \leq \underline{R}<\bar{R}$. The ratio of $\bar{R}$-types is $\rho \in(0,1)$. Sellers observe buyers' valuations at the price-setting stage.20

Consider, first, the case in which both sellers and all buyers are located on platform $i$. If, for example, there are $M_{B}=2$ buyers on platform $i$, there are four pairs of willingnessto-pay that the sellers can encounter: $(\underline{R}, \underline{R}),(\underline{R}, \bar{R}),(\bar{R}, \underline{R})$ and $(\bar{R}, \bar{R})$. For any pair with fewer $\bar{R}$-type buyers than sellers, the unique equilibrium is that sellers set $p^{*}=\underline{R}$ because of Bertrand competition. Only if there are at least as many $\bar{R}$-type buyers as sellers-i.e $(\bar{R}, \bar{R})$ realizes - there is the unique equilibrium that sellers set $p^{*}=\bar{R}$. The probability of this event equals $\rho^{2}$. The expected profit of each seller is then $\pi(2,2)=\rho^{2} \bar{R}+\left(1-\rho^{2}\right) \underline{R}$. More generally, denoting the probability that the number of high-type buyers is larger

\footnotetext{
${ }^{20}$ This simplifying assumption implies that sellers can observe whether or not they are located in a market with sufficiently many $\bar{R}$-type buyers when they set their prices. Yet, in general, it suffices for our argument that prices are increasing in the number of buyers.
} 
than the number of sellers (i.e., $\operatorname{Pr}\left\{\sharp_{\left\{R_{l}=\bar{R}\right\}_{l=1}^{M_{B}}} \geq 2\right\}$ ), by $Q\left(M_{B}, 2\right)$, we obtain

$$
Q\left(M_{B}, 2\right)=\sum_{k=2}^{M_{B}}\left(\begin{array}{c}
M_{B} \\
k
\end{array}\right) \rho^{k}(1-\rho)^{M_{B}-k} .
$$

The expected profit can then be written as $\pi\left(M_{B}, 2\right)=Q\left(M_{B}, 2\right) \bar{R}+\left(1-Q\left(M_{B}, 2\right)\right) \underline{R}$, which corresponds to $\pi^{d}$ of the baseline model.

If, instead, one seller per category locates on platform $A$ and the other on platform $B$, and each buyer joins with probability $1 / 2$ platform $A$ and with probability $1 / 2$ platform $B$ (which will happen in equilibrium), the expected profit of a seller on platform $i$ is $\pi(l, 1)=Q(l, 1) \bar{R}+(1-Q(l, 1)) \underline{R}$ if $l \in\left\{1, \ldots, M_{B}\right\}$ buyers join platform $i$, as $q(l, 1)$ is the probability that there is at least one $\bar{R}$-type buyer among the $l$ buyers. The probability that $l \in\left\{1, \ldots, M_{B}\right\}$ buyers locate on platform $i$ is given by

$$
P(l)=\left(\begin{array}{c}
M_{B} \\
l
\end{array}\right)\left(\frac{1}{2}\right)^{l}\left(\frac{1}{2}\right)^{M_{B}-l} .
$$

Overall, the expected profit of a single seller located on a platform can then be written as $\sum_{l=1}^{M_{B}} P(l) \pi(l, 1)$. This expression corresponds to $\pi^{m} / 2$ - that is, the monopoly profit of a seller when reaching each buyer with probability $1 / 2$. Table 2 illustrates how the ratio $\pi^{d} / \pi^{m}$ depends on the number of buyers per category. (Note that $\pi^{d}$ and $\pi^{m}$ depend on the number of buyers $M_{B}$.)

\begin{tabular}{|r|cccccccccc|}
\hline$M_{B}$ & 1 & 2 & 3 & 4 & 5 & 6 & 7 & 8 & 9 & 10 \\
\hline$\pi^{d} / \pi^{m}$ & 0 & 0.1333 & 0.2367 & 0.3162 & 0.3769 & 0.4228 & 0.4570 & 0.4821 & 0.5002 & 0.5129 \\
\hline
\end{tabular}

The ratio $\pi^{d} / \pi^{m}$ as a function of the number of buyers $M_{B}$ per category and parameter values $\rho=1 / 4$, $\underline{R}=0$ and $\bar{R}=1$.

Table 2: Thin Markets

Example 4: Linear Cournot competition with fixed costs per buyer.

Consider a Cournot model with linear inverse demand $p\left(q_{1}+q_{2}\right)=\alpha-\beta\left(q_{i}+q_{-i}\right)$ and constant marginal costs $c>0$, with $\alpha>c, \beta>0$, and $i=1,2$. In addition to their marginal cost, sellers incur a fixed $\operatorname{cost} F>0$ per buyer. The duopoly equilibrium profit per buyer is $\pi^{d}=\max \left\{(\alpha-c)^{2} /(9 \beta)-F, 0\right\}$. For a monopolist, the inverse demand is $p\left(q^{m}\right)=\alpha-\beta q^{m}$, and the monopoly profit per buyer is $\pi^{m}=\max \left\{(\alpha-c)^{2} /(4 \beta)-F, 0\right\}$. If both profits are strictly positive, the ratio $\pi^{d} / \pi^{m}$ is

$$
\frac{4\left[(\alpha-c)^{2}-9 \beta F\right]}{9\left[(\alpha-c)^{2}-4 \beta F\right]},
$$

which is always less than $1 / 2$. 


\section{Segmentation versus Agglomeration}

In this section, we characterize the equilibrium of the 4-stage game. In particular, we provide conditions for segmentation or agglomeration to be an equilibrium outcome.

In Section 2.2, we analyzed stages 3 and 4 . We now turn to the location decisions of buyers and sellers in stage 2 . Here, multiple Nash equilibria may exist, given the listing fees set by platforms in the first stage. We first determine the set of Nash equilibria in stage 2 . We then explain how our equilibrium selection criteria ensure a unique prediction. A detailed analysis is provided in Appendix A.

Suppose, for example, that listing fees $f_{A}$ and $f_{B}$ are close to zero. Then, it is a Nash equilibrium that all sellers and all buyers are active on only one platform. In such an agglomeration equilibrium on platform $i$, a seller's profit is $\pi^{d}-f_{i}$ and a buyer's utility is $V^{d}$. In addition to two such agglomeration equilibria, there is also a segmentation equilibrium, in which the sellers in each category locate on different platforms, and half of the buyers are active on platform $A$ and the other half on platform $B$. Under segmentation, the profit of a seller joining platform $i$ is $\pi^{m} / 2-f_{i}$, whereas the utility of a buyer is $V^{m}$.

If, instead, both platforms' listing fees were higher than $\max \left\{\pi^{d}, \pi^{m} / 2\right\}$, sellers would make losses in an agglomeration and a segmentation equilibrium. In this case, as long as each platform charges a fee below $\pi^{m}$, there exist two equilibria in which only one seller is active in each category, either on platform $A$ or on platform $B$, and all buyers use this platform. We call an equilibrium of this type stand-alone equilibrium.

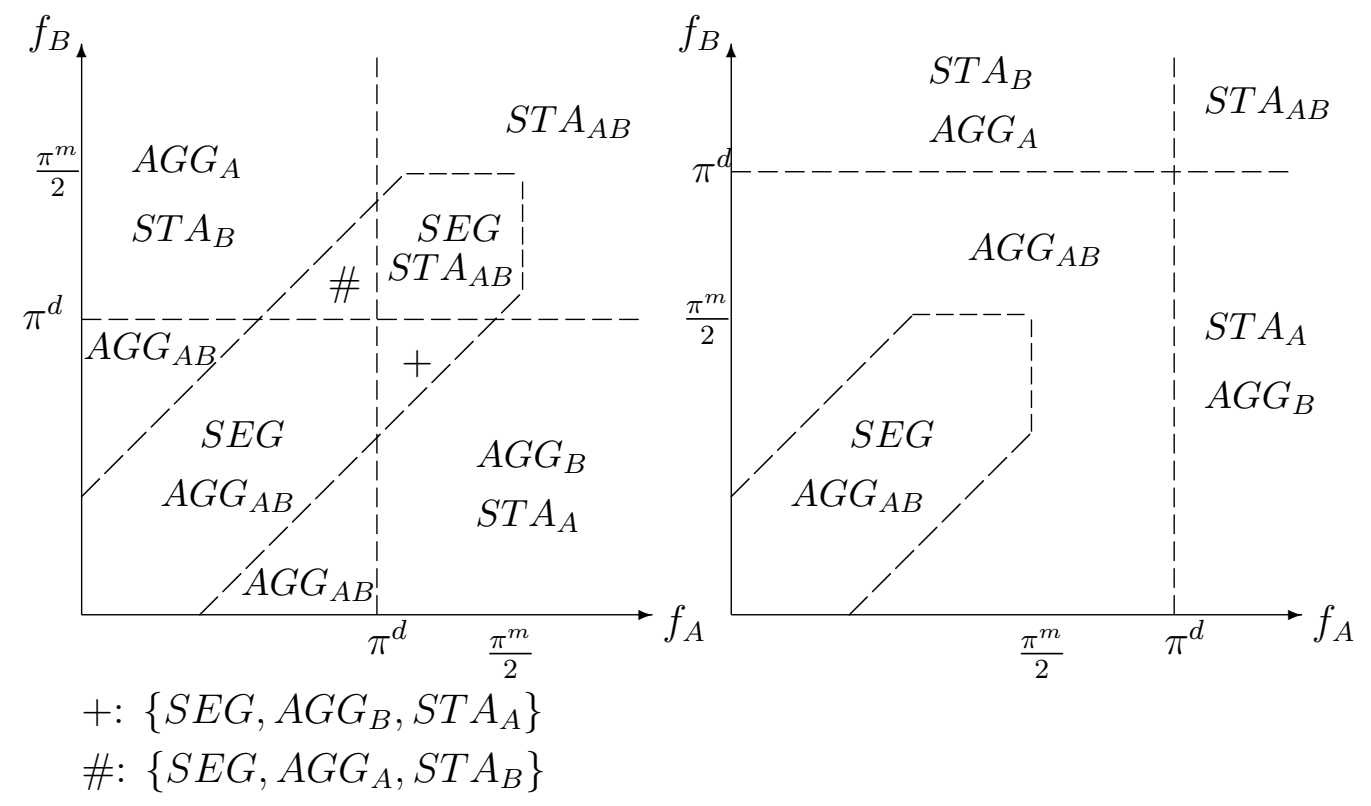

Figure 1: Possible equilibrium configurations at stage $2: \pi^{d} / \pi^{m}<1 / 2$ on the left-hand side and $\pi^{d} / \pi^{m} \geq 1 / 2$ on the right-hand side 
The set of Nash equilibria is visualized in Figure 1-we focus on the relevant case $\left\{f_{A} \leq \pi^{m}, f_{B} \leq \pi^{m}\right\}$ because a fee above $\pi^{m}$ leads to zero demand and, in equilibrium of the full game, no platform will set such a fee. The left panel of the figure displays the case $\pi^{d} / \pi^{m}<1 / 2$, whereas the right panel displays the opposite case. In the figure, the agglomeration equilibrium on platform $i$ is denoted by $A G G_{i}$ (and by $A G G_{A B}$ if an agglomeration equilibrium on either platform exists); the stand-alone equilibrium is denoted by $S T A_{i}$; and the segmentation equilibrium is denoted by $S E G$. As can be seen in the left panel, there are regions in which three equilibrium configurations coexist.

We turn to the equilibrium selection accomplished through our refinement, illustrated in Figure 2. First, applying the concept of coalition-proofness eliminates the multiplicity of agglomeration equilibria off the diagonal. The reason is that a coalition of sellers and buyers will always choose to be active on the platform with the lower fee. The same reasoning holds if there is a multiplicity of stand-alone equilibria, and if an agglomeration and a stand-alone equilibrium co-exist.

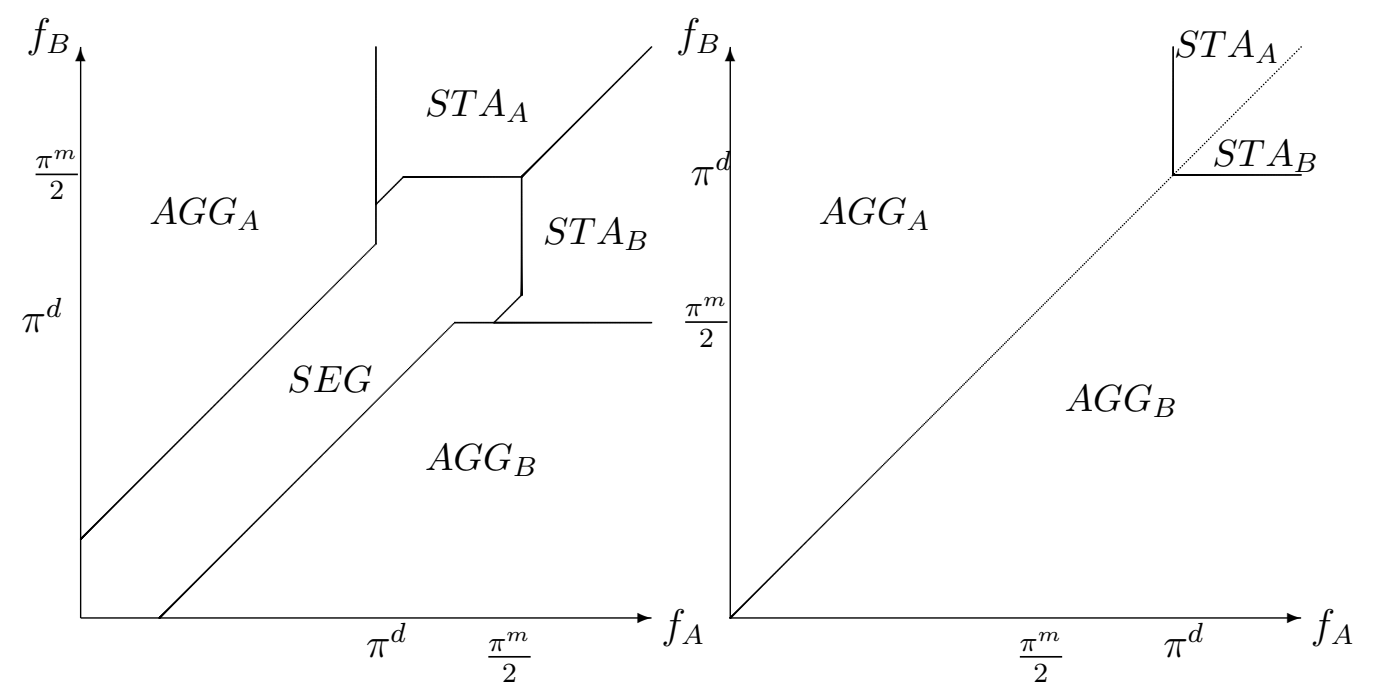

Figure 2: Selected equilibrium configurations at stage 2: $\pi^{d} / \pi^{m}<1 / 2$ on the left-hand side and $\pi^{d} / \pi^{m} \geq 1 / 2$ on the right-hand side

Second, the joint use of coalition-proofness and profit-dominance of sellers also singles out a unique equilibrium type for regions in which the segmentation equilibrium exists together with another type of equilibrium 21 To understand this result, consider, again, the case in which the listing fees of both platforms are close to zero. For $\pi^{d} / \pi^{m} \geq 1 / 2$, the segmentation equilibrium is then not stable to the deviation of a coalition of sellers and buyers who are active on the platform with the higher fee. If this coalition switches to the rival platform, buyers are better off because they observe the offers of all sellers, and sellers are also (weakly) better off because they now serve all buyers instead of only half of

\footnotetext{
${ }^{21}$ In Appendix A, we show that the two refinements are never in conflict with each other.
} 
them. This is profitable because $\pi^{d} \geq \pi^{m} / 2$. Since no subcoalition can gain from further deviation, all segmentation equilibria are eliminated when $\pi^{d} / \pi^{m} \geq 1 / 2$, as can be seen in the right panel of Figure 2. By contrast, if $\pi^{d} / \pi^{m}<1 / 2$, the coalition-proof refinement has no bite, as the deviation is no longer profitable for sellers. Using seller dominance, however, now singles out a unique equilibrium. In particular, if $f_{i}<\pi^{m} / 2-\pi^{d}+f_{-i}$, segmentation is more profitable than agglomeration for sellers. This is displayed in the left panel of Figure 2, which shows that segmentation is the unique equilibrium if $f_{i}$ and $f_{-i}$ are relatively close to each other (and lower than $\pi^{m} / 2$ ).

We turn to platform pricing in the first stage. Although platforms are homogeneous, the Bertrand logic does not necessarily apply in our situation. The reason is that sellers may benefit from segmentation, which implies that a platform does not necessarily attract all sellers and buyers when undercutting the rival's fee. The next four propositions characterize the equilibrium listing fees for all parameter ranges and provide precise conditions for platforms to sustain positive fees.

If the ratio of duopoly to monopoly profits is large (i.e., $\pi^{d} / \pi^{m} \geq 1 / 2$ ), agglomeration occurs - we do not select between the two payoff-equivalent agglomeration equilibria. From a seller's point of view, the effect that agglomeration reduces profits due to competition is dominated by the demand expansion effect that all buyers (instead of only half of them) observe the seller's offer. Since each platform receives the entire demand by setting a fee lower than its rival's, platforms "play hard" and fight fiercely to become dominant. Thus, in this region, the standard Bertrand argument applies, and homogeneous platforms charge fees equal to marginal cost in equilibrium.

Proposition 1. Agglomeration. If $\pi^{d} / \pi^{m} \geq 1 / 2$, in equilibrium, the listing fees are $f_{A}^{\star}=f_{B}^{\star}=0$, and platforms' profits are $\Pi_{A}^{\star}=\Pi_{B}^{\star}=0$.

By contrast, if the ratio of duopoly to monopoly profits is small (i.e., $\pi^{d} \leq 1 / 4 \pi^{m}$ ), segmentation occurs. Sellers avoid competition by choosing to locate on different platforms, which, in turn, is exploited by platforms. To see this, suppose that both platforms charge a fee of zero. If $\pi^{d}$ is lower than $\pi^{m} / 2$, sellers choose to segment. But then a platform can raise its fee slightly without reducing its demand. Thus, the platform with the higher fee remains active and raises strictly positive profits.

Proposition 2. Segmentation. If $\pi^{d} / \pi^{m} \leq 1 / 4$, in the unique equilibrium, the listing fees are $f_{A}^{\star}=f_{B}^{\star}=\pi^{m} / 2$, and platform profits' are $\Pi_{A}^{\star}=\Pi_{B}^{\star}=\pi^{m} / 2$.

The proposition shows that platforms not only obtain a strictly positive profit, but even extract the entire surplus from sellers. The argument is as follows. If a platform deviates from the equilibrium listing fee $f_{i}^{\star}=\pi^{m} / 2$ to a listing fee slightly below $\pi^{d}$, this induces sellers and buyers to agglomerate on the deviating platform. The deviant 
platform then obtains a profit of $2 \pi^{d}$. Instead, the equilibrium profit is $\pi^{m} / 2$, which is larger than $2 \pi^{d}$ if $\pi^{d} / \pi^{m} \leq 1 / 4$. Hence, no platform has an incentive to deviate from the listing fee $\pi^{m} / 2$ - platforms "play soft" and do not fight for the dominant position. To sum up, if competition between sellers is sufficiently intense, platforms obtain positive profits by inducing sellers to segment the market. Interestingly, fierce competition among sellers enables platforms to sustain high profits in equilibrium.

In the intermediate range $1 / 4<\pi^{d} / \pi^{m}<1 / 2$, platforms randomize over listing fees. The intuition for the non-existence of a pure-strategy equilibrium in this range is as follows: For any fee set by platform $i$, platform $-i$ 's best response is to either set a fee that is lower by a discrete amount to induce agglomeration or to set a fee that is higher by a discrete amount leading to segmentation. This creates a cycle in best responses. Suppose that platform $i$ sets a relatively high fee. Platform $-i$ 's best response is then to set a lower fee, so as to just induce agglomeration. The best response of platform $i$ is to lower its fee slightly and induce segmentation again. This sequence of best responses continues until the fee of platform $i$ reaches such a low level that platform $-i$, instead of setting a lower fee, prefers to set a fee higher than that of platform $i$, so as to just induce segmentation. In turn, platform $i$ 's best response is to reduce its fee slightly to induce agglomeration, and so on. Therefore, the sequence continues and does not converge.

The logic behind the mixed-strategy equilibrium in the range $1 / 4<\pi^{d} / \pi^{m}<1 / 2$ is reminiscent of, but distinct from, Bertrand-Edgeworth cycles. In the latter, the bestresponse dynamic involves a marginal undercutting of the rival's fee, as long fees are sufficiently high (see, for example, Edgeworth, 1925; Maskin and Tirole, 1988). By contrast, in our model, for any fee charged by the rival, the best response is to set a fee that is higher or lower by a discrete amount. ${ }^{22}$ In fact, the range of subscription fees over which platforms mix can be divided into two intervals, a lower and an upper one. In the lower interval, fees are set with the intention to induce agglomeration. In the upper interval, fees are set with the intention to induce segmentation. This leads to mass points in the mixing distribution and potentially disjoint mixing sets.

In the region of $3 / 8 \leq \pi^{d} / \pi^{m}<1 / 2$, the upper bound of the lower interval in which a platform aims to induce agglomeration coincides with the lower bound of the upper interval in which a platform aims to induce segmentation. This implies that platforms randomize over a convex set.

Proposition 3. Probabilistic segmentation and agglomeration with listing fees chosen from a convex set. If $3 / 8 \leq \pi^{d} / \pi^{m}<1 / 2$, there is a unique mixed-strategy equilibrium, in which platforms set fees in the domain $f_{i} \in\left[\pi^{m}-2 \pi^{d}, 2 \pi^{m}-4 \pi^{d}\right]$. The mixing probability

\footnotetext{
${ }^{22}$ In this respect, our equilibrium also differs from those found in papers in the search literature, such as Varian (1980) or Janssen and Moraga-González (2004).
} 
is characterized by the cumulative distribution function

$$
G_{1}(f)= \begin{cases}\frac{f-\left(\pi^{m}-2 \pi^{d}\right)}{f+1 / 2\left(\pi^{m}-2 \pi^{d}\right)}, & \text { if } f \in\left[\pi^{m}-2 \pi^{d}, 3 / 2 \pi^{m}-3 \pi^{d}\right) ; \\ \frac{2 f-5 / 2\left(\pi^{m}-2 \pi^{d}\right)}{f-1 / 2\left(\pi^{m}-2 \pi^{d}\right)}, & \text { if } f \in\left[3 / 2 \pi^{m}-3 \pi^{d}, 2 \pi^{m}-4 \pi^{d}\right],\end{cases}
$$

with a mass point at $f=3 / 2 \pi^{m}-3 \pi^{d}$, which is chosen with probability $1 / 4$. The expected profit is $\Pi_{A}^{\star}=\Pi_{B}^{\star}=3 \pi^{m} / 2-3 \pi^{d}$.

The cumulative distribution function $G_{1}(f)$ is illustrated in Figure 3. The mass point is at the fee that separates the two intervals. Therefore, setting such a fee induces segmentation with probability (almost) 1. Since the event that both platforms choose this fee occurs with strictly positive probability, the expected equilibrium profit in this regime must equal $3 / 2 \pi^{m}-3 \pi^{d}$.

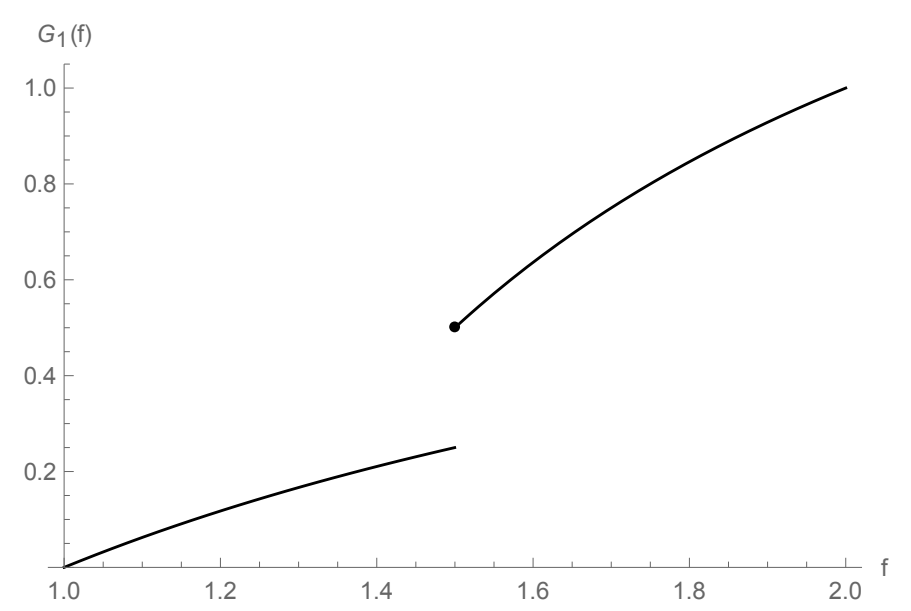

Figure 3: First mixed-strategy equilibrium: Cumulative distribution function with parameters $\pi^{m}=5$ and $\pi^{d}=2$.

The highest fee that platforms can charge to obtain positive demand is $\pi^{m} / 2$. If $\pi^{d} / \pi^{m}$ is at the lower bound of the mixing region of Proposition 3 (i.e., $\pi^{d} / \pi^{m}=3 / 8$ ), the highest fee in the mixing range, $2 \pi^{m}-4 \pi^{d}$, reaches this level. It follows that if $\pi^{d} / \pi^{m}$ is lower, the equilibrium will be different. In particular, as a fee of $\pi^{m} / 2$ must be the upper bound, probability mass will be shifted to this point, and the distribution will entail a mass point at the highest fee. In addition, the best response to this highest fee (i.e., the largest fee in the lower interval) no longer coincides with the fee that induces segmentation with probability (almost) 1. The latter fee is the lowest one in the upper interval, which implies that the support of the mixing region becomes non-convex. This is shown in Proposition 4 .

Proposition 4. Probabilistic segmentation and agglomeration with listing fees chosen from a non-convex set. If $1 / 4<\pi^{d} / \pi^{m}<3 / 8$, there is a unique mixed-strategy equilib- 
rium, in which platforms set fees in the domain $f_{i} \in\left[\pi^{m} / 4, \pi^{d}\right) \cup\left[3 \pi^{m} / 4-\pi^{d}, \pi^{m} / 2\right]$. The mixing probability is characterized by the cumulative distribution function

$$
G_{2}(f)= \begin{cases}\frac{f-1 / 4 \pi^{m}}{f+1 / 2\left(\pi^{m}-2 \pi^{d}\right)}, & \text { if } f \in\left[\pi^{m} / 4, \pi^{d}\right) ; \\ \frac{2 f-1 / 4 \pi^{m}-3 / 2\left(\pi^{m}-2 \pi^{d}\right)}{f-1 / 2\left(\pi^{m}-2 \pi^{d}\right)}, & \text { if } f \in\left[3 \pi^{m} / 4-\pi^{d}, \pi^{m} / 2\right) ; \\ 1, & \text { if } f=\pi^{m} / 2 ;\end{cases}
$$

with two mass points, one at the highest fee in the support $f=\pi^{m} / 2$, which is chosen with probability $\left(2 \pi^{d}-1 / 2 \pi^{m}\right) / \pi^{m}$, and the other at the lower bound of the upper interval $f=3 \pi^{m} / 4-\pi^{d}$, which is chosen with probability $\left(3 / 4 \pi^{m}-2 \pi^{d}\right) / \pi^{d}$. The expected profit is $\Pi_{A}^{\star}=\Pi_{B}^{\star}=3 \pi^{m} / 4-\pi^{d}$.

Figure 4 illustrates $G_{2}(f)$ in the second mixing regime. The support of the distribution is $[5 / 4,7 / 4) \cup[2,5 / 2]$. The intuition for the lower mass point (at $f=3 \pi^{m} / 4-\pi^{d}$ ) is the same as the one given in the first mixed regime. The intuition for the mass point at $f=\pi^{m} / 2$ is, as explained above, that $\pi^{m} / 2$ is an upper bound in any mixing equilibrium.

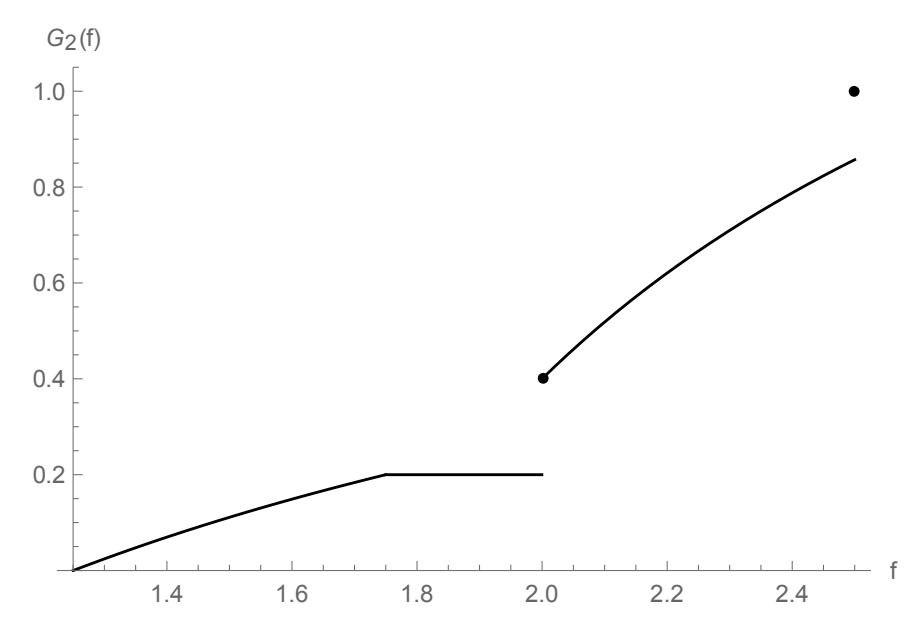

Figure 4: Second mixed-strategy equilibrium: Cumulative distribution function with parameters $\pi^{m}=5$ and $\pi^{d}=7 / 4$.

As can be seen from Proposition 4, the gap between the two intervals widens as $\pi^{d}$ falls. In the limit, as $\pi^{d} \rightarrow \pi^{m} / 4$, all probability mass is on $\pi^{m} / 2$. Therefore, the equilibrium is continuous. As $\pi^{d}$ falls, expected fees rise continuously, as do platforms' profits. The expected equilibrium platform profit is a continuous function but has three kinks at the boundary points of the regions (see Figure 5).

From the analysis, it is easy to see that the assumption of buyers splitting evenly on platforms when being indifferent is not crucial for the results. If this split is more in favor of platform $i$, the pure-strategy segmentation equilibrium exists for a smaller range: as this equilibrium is less attractive for platform $-i$, the platform has a stronger deviation 


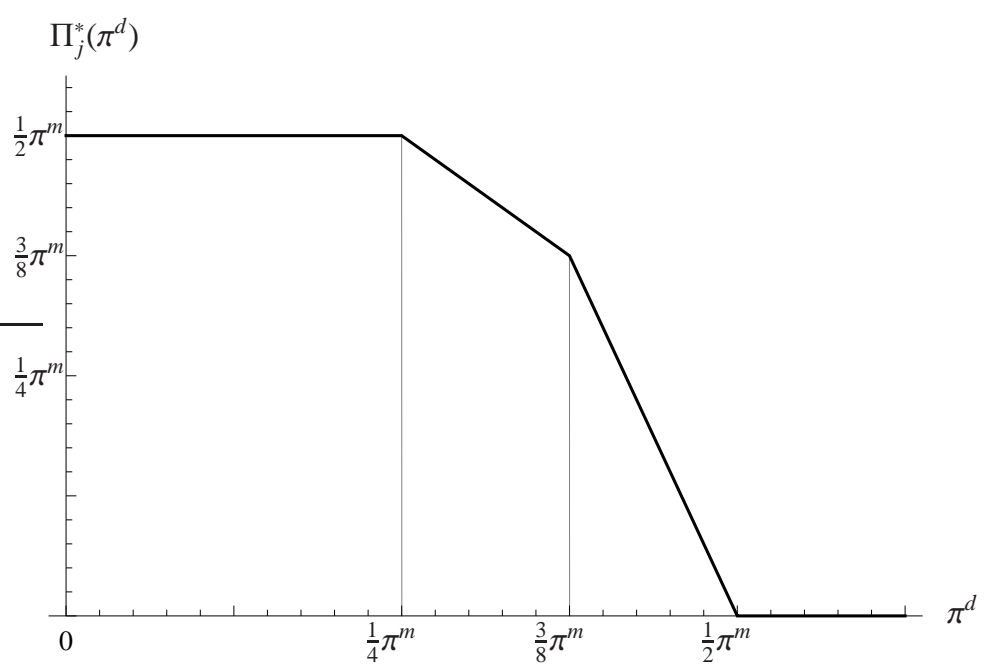

Figure 5: Expected platform profit $\Pi_{j}^{\star}$ as a function of $\pi^{d}$.

incentive. However, if $\pi^{d}$ is sufficiently small, deviation is not profitable for platform $-i$ because the platform can obtain only a low profit with agglomeration. Therefore, an asymmetric segmentation equilibrium still occurs.

Evaluating the different equilibrium regions from a welfare perspective includes buyers' utilities. There are two reasons that the segmentation equilibrium is less efficient than an agglomeration equilibrium. First, because buyers are not informed about all offers, they may buy products with a greater mismatch cost than when they are informed about all offers. Second, if $p^{m}>p^{d}$, the inefficiency in the product market due to market power of sellers is higher, implying that the quantities bought by buyers in a segmentation equilibrium are lower than in an agglomeration equilibrium. By contrast, platforms enjoy profits when they induce segmentation with positive probability, and the resulting market structure is inefficient. In Section 6, we discuss some policy implications that arise from our analysis.

Examples. We express the equilibrium regions in terms of the underlying parameters describing seller competition in the examples laid out in the previous section. In Example 1, which features price competition and Hotelling demand, a larger degree of product differentiation $t$ increases the sufficient statistic $\pi^{d} / \pi^{m}$. We obtain that the agglomeration region applies for $2(v-c) / 3 \geq t \geq(v-c) / 2$, the first mixing region if $(v-c) / 2>t \geq$ $3(v-c) / 7$, the second mixing region if $3(v-c) / 7>t>(v-c) / 3$, and the segmentation region if $t \leq(v-c) / 3$.

In Example 2, which features price competition and a representative consumer, the boundaries of the regions are affected by $\gamma \in[0, \beta)$, with a higher $\gamma$ implying less differentiation and fiercer competition. The agglomeration region applies approximately for $\gamma \leq 0.62 \beta$, the first mixing region for $0.62 \beta<\gamma \leq 0.74 \beta$, the second mixing region for 
$0.74 \beta<\gamma<0.85 \beta$, and the segmentation region for $\gamma \geq 0.85 \beta$.

In Example 3, which features thin markets, the number of buyers and the probability for a buyer being of high valuation are the drivers for the platform market structure. With two buyers and $\underline{R}=0$, agglomeration occurs if the probability of high-type buyers is $\rho \geq 4 / 5$, whereas segmentation occurs if $\rho \leq 4 / 9$. A larger probability for the high type reduces competition, as it is more likely that both sellers face a high-type buyer, which leads to an increase in $\pi^{d}$. Regarding the number of buyers, in our numerical example with $\rho=1 / 4$ and $\underline{R}=0$, agglomeration occurs when there are more than eight buyers (cf. Table 2), whereas segmentation occurs for three or less buyers. The first mixing region prevails for five to eight buyers and the second mixing region for four buyers. When the probability of the high type decreases, the boundaries for all regions shift upward, which implies that segmentation becomes more likely.

In Example 4, which features Cournot competition and fixed costs per buyer $F$, a higher $F$ favors segmentation, as a seller serves a smaller mass of buyers in this configuration. Accordingly, we obtain that the segmentation region applies if $(a-c)^{2} /(4 b)>$ $F \geq 7(a-c)^{2} /(108 b)$ - that is, if fixed costs are sufficiently high (but lower than $\pi^{m}$ ). The second mixing region applies for $(a-c)^{2} /(36 b)<F<7(a-c)^{2} /(108 b)$, the first mixing region for $0 \leq F \leq(a-c)^{2} /(36 b)$, and the agglomeration region does not exist (due to the fact that $\pi^{d} / \pi^{m}<1 / 2$ ).

Finally, we note that, as has been demonstrated in the example with thin markets, our results do not rely on a constant per-buyer profit. If this profit was not constant, the equilibrium characterization would be more involved, as the boundaries of the regions then depend on the mass of buyers on each platform in addition to the profit per buyer. However, under standard regularity assumptions on demand, the qualitative results are the same as in our analysis.

\section{Multi-Homing}

In the baseline model, we focus on the case in which both buyers and sellers are singlehoming. In this section, we consider multi-homing of either buyers or sellers. We will show that in both cases, our qualitative results remain.

\subsection{Multi-Homing of Buyers}

Assume that a fraction $\alpha \in[0,1]$ of buyers joins both platforms. A natural reason is that buyers incur time cost to be active on a second platform, and they are heterogeneous with respect to these time costs. Therefore, only buyers with sufficiently low time costs are active on both platforms. A higher $\alpha$ can then be interpreted as a reduction in time 
costs 23

Multi-homing of buyers affects the sellers' profits. In fact, a seller will never obtain the monopoly profit because a fraction $\alpha$ of buyers is informed about both offers. In a segmentation equilibrium, half of the single-homing buyers are active on platform $A$ and the other half on platform $B$. Because there is a mass $1-\alpha$ of single-homing buyers, each platform has a total buyer mass of $(1+\alpha) / 2$, out of which $(1-\alpha) / 2$ are single-homers and $\alpha$ are multi-homers. As sellers do not know which buyer single-homes and which one multi-homes, they set a single price in the product market. The equilibrium price will depend on $\alpha$ because multi-homers' demand may differ from single-homers' demand. Therefore, we can write the expected profit that a seller obtains from a buyer as $\pi(\alpha)$. In particular, $\pi(0)=\pi^{m}$ and $\pi(1)=\pi^{d}$. Naturally, $\pi^{\prime}(\alpha) \leq 0$, which implies that for all $\alpha \in[0,1], \pi(\alpha) \in\left[\pi^{d}, \pi^{m}\right]$ [4 $^{24}$ Below, we will show how a change in $\alpha$ plays out in Example 1. The equilibrium with multi-homing buyers is characterized by the following proposition.

Proposition 5. All results of Propositions 1 through 4 carry over to the case of buyer multi-homing, after replacing $\pi^{m} / 2$ by

$$
\pi(\alpha) \frac{1+\alpha}{2}
$$

The proposition shows that the qualitative results of the previous section remain valid if buyers can multi-home. Although segmentation does not give sellers monopoly power, it nevertheless lowers the competitive pressure because some buyers are still informed only about one seller's offer, and platforms will exploit this.

The question arises: Do platforms benefit from buyer multi-homing? If we are in the range of the agglomeration equilibrium, nothing changes compared to buyer singlehoming because platforms are engaged in Bertrand competition. However, this is not true for the regions in which the segmentation equilibrium occurs with positive probability. There are two countervailing forces. First, platforms have more buyers, which leads to a larger demand per seller. In fact, instead of serving a buyer mass of $1 / 2$ (as with singlehoming), platforms now have a mass of $(1+\alpha) / 2$ of buyers. This allows platforms to charge higher listing fees. However, the countervailing force is that sellers make smaller profits in the product market because some buyers are informed about both offers. The per-buyer profit is then $\pi(\alpha)<\pi^{m}$. It follows that platforms are hurt by the possibility of buyer multi-homing if the competition effect dominates the demand-enhancing effect.

\footnotetext{
${ }^{23}$ For example, if a distribution of time costs among buyers first-order stochastically dominates another one, the latter distribution leads to a higher fraction $\alpha$ of multi-homing buyers.

${ }^{24}$ In an agglomeration equilibrium, a seller's profit is unchanged since all buyers see both offers. This leads to a profit of $\pi^{d}$ for each seller.
} 
We illustrate this result with the help of Example 1. With Hotelling demand, we obtain

$$
\pi(\alpha) \frac{1+\alpha}{2}=\left\{\begin{array}{ccc}
\frac{(\alpha t+(v-c)(1-\alpha))^{2}}{2 t(2-\alpha)^{2}} & \text { if } & t \geq \frac{v-c}{2} \\
\frac{v-c-t}{2} & \text { if } & \frac{\alpha(v-c)}{1+\alpha} \leq t<\frac{v-c}{2} \\
\frac{t}{2 \alpha} & \text { if } & t<\frac{\alpha(v-c)}{1+\alpha}
\end{array}\right.
$$

Comparing $\pi(\alpha)(1+\alpha) / 2$ with $\pi^{m} / 2$, the former is larger than the latter for high $t$, but the reverse holds true for low $t$. In particular, for $t \geq(v-c) / 2$, the demand-enhancing effect of multi-homing prevails, as product market competition is not particularly fierce. However, in this region, $\pi^{d}$ is larger than $\pi(\alpha)(1+\alpha) / 2$, which implies that platforms in equilibrium still set fees equal to zero and an agglomeration equilibrium emerges. Therefore, although the effect of increased demand dominates, this does not affect the outcome. If, instead, $t$ is small (i.e., $t<\alpha(v-c) /(1+\alpha)$ ), multi-homing lowers sellers' segmentation profit, as competition is fierce, and, therefore, lowers the listing fees that platforms charge in equilibrium. This effect is also dominant in the intermediate region, implying that platforms obtain weakly lower profits with buyer multi-homing.

Finally, we observe that in a segmentation equilibrium multi-homing consumers exert a positive externality on single-homing ones. As product prices are lower with more multi-homing consumers, single-homers benefit as well. This implies that devices which foster multi-homing, such as metasearch engines, also benefit consumers who do not use them.

\subsection{Multi-Homing of Sellers}

In this section, we consider the effects of multi-homing of sellers. We focus on a situation with uniform pricing, that is, a multi-homing seller sets the same price on both platforms. Towards the end of the section, we show that the analysis with price discrimination is a special case of that with uniform pricing and our main insight is robust.

In contrast to buyers, sellers need to pay for being active on a platform. Therefore, even without any exogenous costs for using a second platform, sellers do not necessarily find it profitable to multi-home 25 We are particularly interested to understand the conditions under which seller multi-homing affects the platform market structure and whether platforms benefit. This focus is different from the literature on two-sided pricing in two-sided markets, which has investigated the effect of seller multi-homing on the price structure, pointing out that that platforms exert monopoly power on the multi-homing side (see, e.g., Armstrong, 2006, or Hagiu, 2006). In that literature, the platform market structure is given.

\footnotetext{
${ }^{25}$ Introducing such listing costs would not change the main result.
} 
With multi-homing sellers, new potential equilibrium configurations in the second stage may occur. First, both sellers in a category may multi-home. In that case, all buyers are exposed to both offers, implying that each seller receives the duopoly profit $\pi^{d}$ per buyer. But the profit per buyer is then equivalent to the profit when both sellers agglomerate on one platform. In the latter case, however, sellers have to pay only one listing fee. Therefore, the configuration in which both sellers multi-home is never coalition-proof and will not occur in equilibrium under our refinement.

Second, a configuration is possible in which one seller in a category single-homes and the other one multi-homes - a situation we refer to as partial multi-homing. If in one half of the categories, the single-homing seller is on platform $A$ and in the other half on platform $B$, buyers are indifferent between both platforms, as the same number of sellers are active on either platform. Buyers are, therefore, willing to split evenly between the platforms. In this situation, competition in the product market is asymmetric. Half of buyers are active on the platform in which only the multi-homing seller is present and observe only the offer of this seller. The other half observes the offers of both sellers. Let us denote the per-buyer profit of the multi-homing seller by $\pi^{M H}$ and that of the single-homing seller by $\pi^{S H}$. As the multi-homing seller can act as a monopolist to one half of the buyers but faces competition for the other half, its price $p^{M H}$ will be between $p^{m}$ and $p^{d}$. The same holds for the price of the single-homing seller $p^{S H}$, as this seller faces competition from a rival who has some 'captive' buyers and will, therefore, price higher than in duopoly competition. For the sellers' profits, we assume that

$$
\pi^{d} \leq \pi^{S H} \leq \pi^{M H} \leq \pi^{m}
$$

which follows from the sellers' pricing decisions. These relations are compatible with our specific examples.

We can now establish the equilibrium with multi-homing sellers.

Proposition 6. $\quad$ For $\pi^{d} / \pi^{m} \geq 1 / 2$ and $\pi^{d} / \pi^{m} \leq 1 / 4$, the equilibrium is the same as the one characterized in Propositions 1 and 2 , respectively.

- For $3 / 8 \leq \pi^{d} / \pi^{m}<1 / 2$, the equilibrium is the same as the one characterized in Proposition 3 if $\pi^{M H} \leq 3 / 2 \pi^{m}-2 \pi^{d}$.

Similarly, for $1 / 4<\pi^{d} / \pi^{m}<3 / 8$, the equilibrium is the same as the one characterized in Proposition 4 if $\pi^{M H} \leq 3 / 4 \pi^{m}$.

- Instead, for (i) $3 / 8 \leq \pi^{d} / \pi^{m}<1 / 2$ and $\pi^{M H}>3 / 2 \pi^{m}-2 \pi^{d}$ and for (ii) $1 / 4<$ $\pi^{d} / \pi^{m}<3 / 8$ and $\pi^{M H}>3 / 4 \pi^{m}$, respectively, in equilibrium, platforms set fees of $f_{A}^{\star}=f_{B}^{\star}=0$, and sellers play an agglomeration equilibrium if $\pi^{d}>\pi^{S H} / 2$ and a partial multi-homing equilibrium if $\pi^{d} \leq \pi^{S H} / 2$. 
The proposition shows that for some parameter constellations, the equilibrium derived in Propositions 1 to 4 remains unchanged. Foremost, if competition between sellers is relatively fierce, the segmentation equilibrium still exists. Although sellers can multihome, doing so would reduce their profits by too large an amount; hence, they prefer segmentation. Platforms exploit this by extracting the entire seller surplus. Therefore, our insight that segmentation leads to high platform profits, even though platforms are homogeneous, is robust to seller multi-homing.

The proposition also shows that the mixed-strategy equilibrium, which involves segmentation with some probability and features positive platform profits, emerges for a smaller parameter range than in the case of single-homing sellers. It is replaced by an equilibrium in which platforms charge zero listing fees. Thus, we obtain the unambiguous result that platforms set (weakly) lower fees to sellers and earn (weakly) lower profits if the latter can multi-home instead of single-home. This contrasts with the results of the existing literature on two-sided markets, which finds that platforms exert monopoly power on the seller side and, in equilibrium, may set higher fees to sellers and earn higher profits under seller multi-homing.

The intuition behind our result is as follows: If sellers can multi-home, segmentation may break down because sellers have an additional deviation possibility from the segmentation equilibrium. Instead of being active only on the other platform, they can now join both platforms. This deviation is particularly profitable if $\pi^{M H}$ is large. In fact, as can be seen in the proposition, segmentation is more likely to break down if $\pi^{M H}$ is high. As a result, platforms can no longer charge high fees and exploit the possibility that they grant monopoly power to sellers. The homogeneity of the platforms then drives fees and profits down to zero.

Interestingly, this also implies that agglomeration is more likely if sellers can multihome. The general notion in the antitrust economics of platform markets is that multihoming reduces the risk of market tipping because it is more likely that multiple platforms will obtain positive demand (see, e.g., Evans and Schmalensee, 2007) ${ }^{26}$ In our model, a different mechanism is at work - that is, the possibility of multi-homing can break the segmentation equilibrium in which multiple platforms are active.

In addition, (partial) multi-homing occurs in equilibrium under some conditions. ${ }^{27}$ In particular, if $\pi^{S H}$ and $\pi^{M H}$ are relatively large, neither the single-homing nor the multi-homing seller has an incentive to deviate to an agglomeration or a segmentation equilibrium. The partial multi-homing equilibrium is in between pure agglomeration and

\footnotetext{
${ }^{26}$ The German Federal Cartel Office used a similar argument in the clearing of a merger in the housing platform market (Bundeskartellamt, 2016).

${ }^{27}$ Partial multi-homing of sellers can often be observed on price comparison websites. Whereas some sellers list their offers on several platforms at the same time, others use only one.
} 
pure segmentation and consists of elements of both equilibria. While buyers segment, half of them are still informed about both offers due to the multi-homing of one seller in each category. In contrast to the pure segmentation equilibrium, platforms cannot exploit this in equilibrium. As stated in the proposition, the partial multi-homing equilibrium leads to zero fees for platforms. The intuition is similar to the one developed for the agglomeration equilibrium: When slightly undercutting the listing fee of the rival, a platform can get the single-homing seller in each category (and not only in one half of the categories). This leads to an agglomeration equilibrium on the platform with the lower fee, which gives this platform an upward jump in demand, and is, therefore, always profitable. Hence, the standard Bertrand logic applies and drives fees to marginal cost.

We illustrate the result with Example 1. For Hotelling demand, $\pi^{M H}$ and $\pi^{S H}$ are

$$
\pi^{M H}=\frac{(t+v-c)(8 t-(v-c))}{8 t} \text { and } \pi^{S H}=\frac{(v-c)^{2}}{8 t}
$$

in the relevant region. Determining the critical value of the transportation costs so that condition (i) of the third part of Proposition 6 is fulfilled, we obtain that this holds if $(v-c) / 2 \geq t>(3+\sqrt{57})(v-c) / 24 \approx 0.44(v-c)$. Condition (ii) is never fulfilled. As a result, we find that with single-homing of sellers, the mixing region was valid for $(v-c) / 2 \geq t>(v-c) / 3$, whereas with multi-homing, it shrinks to the range $0.44(v-c) \geq t>(v-c) / 3$. In addition, the condition $\pi^{d}<\pi^{S H} / 2$ is not satisfied with Hotelling demand in the relevant range. This implies that a partial multi-homing equilibrium does not exist. If fees are zero, an agglomeration equilibrium will always emerge. 28

We assumed that a multi-homing seller sets the same price on each platform. If price discrimination were possible, the seller would set $p^{d}$ on the platform where the rival is also present and $p^{m}$ on the platform where the seller is in a monopoly position. Using the notation above, this implies that $\pi^{M H}=1 / 2\left(\pi^{d}+\pi^{m}\right)$ and $\pi^{S H}=\pi^{d}$. Therefore, the situation with price discrimination can be analyzed as a special case of the situation analyzed above. It is easy to see that the qualitative results of Proposition 6 still hold.

Finally, we note that although we analyzed multi-homing of buyers and sellers separately, a combination of the two will lead to similar insights. In particular, if competition between sellers is fierce, there is always the incentive to segment the market. This will drive sellers away from agglomeration to full or partial segmentation.

\footnotetext{
${ }^{28}$ The non-existence of the partial multi-homing equilibrium is an artifact of the Hotelling model. With general demand and in some of our other examples, such an equilibrium exists.
} 


\section{Generalizations and Robustness}

To convey the main results and the intuitions in the clearest way, in the baseline model, we made some simplifying assumptions about, for example, the platforms' pricing instruments and the number of platforms and sellers. In this section, we generalize the baseline model by considering alternative pricing instruments on the seller side (Section 5.1) and a general number of platforms and sellers (Section 5.2). We show that our results are robust to these extensions. In addition, we also briefly discuss the alternative selection criterion of the buyer-preferred equilibrium (Section 5.3), the case of two-sided pricing (Section 5.4), and some further extensions (Section 5.5).

\subsection{Platform Pricing Instruments}

In the main model, we consider the case in which platforms charge listing fees to sellers. This pricing instrument is the only feasible one if platforms cannot monitor the transaction between buyers and sellers, as is often the case, e.g., in housing markets. However, in other markets, such as the hotel booking industry, monitoring is possible at relatively low costs. As a consequence, booking services or marketplaces, such as Amazon marketplace, often charge per-transaction fees or a percentage of the price charged by sellers (revenue-sharing). In this section, we consider these two pricing instruments, as well as the combination of listing fee and per-transaction fee.

\subsubsection{Per-transaction fees}

Suppose that the game is the same as the one laid out in Section 2 but that platforms instead of charging listing fees demand a fee per transaction, denoted by $\phi_{i}, i=A, B$. That is, every time a consumer buys a product from a seller, the seller needs to pay $\phi_{i}$ to the platform. A listing fee constitutes a fixed cost for the seller and, therefore, does not affect the pricing choice in the product market. By contrast, a per-transaction fee increases the marginal cost of each seller, and will affect the price that the seller charges. We denote the resulting duopoly equilibrium price in the product market by $p^{d}\left(\phi_{i}\right)$, with $\partial p^{d}\left(\phi_{i}\right) / \partial \phi_{i}>0$, and the associated demand by $D^{d}\left(\phi_{i}\right)$. The resulting duopoly profit (assuming a constant marginal cost of $c$ ) is $\pi^{d}\left(\phi_{i}\right)=D^{d}\left(\phi_{i}\right)\left(p^{d}\left(\phi_{i}\right)-\phi_{i}-c\right)$, with $\partial \pi^{d}\left(\phi_{i}\right) / \partial \phi_{i} \leq 029$ Similarly, in the monopoly case, the resulting price is $p^{m}\left(\phi_{i}\right)$, with $\partial p^{m}\left(\phi_{i}\right) / \partial \phi_{i} \leq 030$ the demand is $D^{m}\left(\phi_{i}\right)$, and the profit is $\pi^{m}\left(\phi_{i}\right)=D^{m}\left(\phi_{i}\right)\left(p^{m}\left(\phi_{i}\right)-\right.$

\footnotetext{
${ }^{29}$ The inequality in $\partial \pi^{d}\left(\phi_{i}\right) / \partial \phi_{i}$ is only weak because in covered markets (as, for example, in the Hotelling model), an increase in $\phi_{i}$ leads to an increase in the product price by the same amount without affecting equilibrium demand, implying that profits are unchanged.

${ }^{30}$ The weak inequality here is due to the fact that in markets with rectangular demand, the monopoly price is independent of cost.
} 
$\left.\phi_{i}-c\right)$, with $\partial \pi^{m}\left(\phi_{i}\right) / \partial \phi_{i} \leq 0$. We maintain the assumption from the main model that $\pi^{d}\left(\phi_{i}\right) / \pi^{m}\left(\phi_{i}\right) \leq 1$ for all $i$.

In addition, we assume that an increase in the per-transaction fee reduces the monopoly profit by more than the duopoly profit, and the same holds true for the monopoly demand compared to duopoly demand; that is,

$$
\frac{\partial \pi^{m}\left(\phi_{i}\right)}{\partial \phi_{i}} \leq \frac{\partial \pi^{d}\left(\phi_{i}\right)}{\partial \phi_{i}} \leq 0 \text { and } \quad \frac{\partial D^{m}\left(\phi_{i}\right)}{\partial \phi_{i}} \leq \frac{\partial D^{d}\left(\phi_{i}\right)}{\partial \phi_{i}} \leq 0 .
$$

These properties hold in standard oligopoly models, including those in our examples.

We can then solve the model as in the case with listing fees. The details are provided in the proof of Proposition 7 in the Appendix. As we demonstrate there, also with pertransaction fees, our selection criterion singles out a unique type of equilibrium in stage 2. Turning to the full game, with per-transaction fees, platforms cannot extract the full profit from sellers. However, we can formulate the analogue to a listing fee of $\pi^{m} / 2$, which is the highest profit a platform can make in a segmentation equilibrium. With per-transaction fees, we denote by $\phi^{m} \equiv \arg \max _{\phi_{i}} \phi_{i} D^{m}\left(\phi_{i}\right) / 2$. We obtain the following result:

Proposition 7. If $\pi^{d}(0) / \pi^{m}(0) \geq 1 / 2$, in equilibrium, both platforms set $\phi_{A}^{\star}=\phi_{B}^{\star}=0$, and buyers and sellers agglomerate on either platform $A$ or platform B. If $\pi^{d}(0) / \pi^{m}(0)<$ $1 / 2$ and

$$
\frac{\phi^{\prime} D^{d}\left(\phi^{\prime}\right)}{\phi^{m} D^{m}\left(\phi^{m}\right)} \leq \frac{1}{4}
$$

where $\phi^{\prime}$ is defined by $\pi^{d}\left(\phi^{\prime}\right)=\pi^{m}\left(\phi^{m}\right) / 2$, in the unique equilibrium, both platforms set $\phi_{A}^{\star}=\phi_{B}^{\star}=\phi^{m}$, and buyers and sellers segment. If $\pi^{d}(0) / \pi^{m}(0)<1 / 2$ and

$$
\frac{\phi^{\prime} D^{d}\left(\phi^{\prime}\right)}{\phi^{m} D^{m}\left(\phi^{m}\right)}>\frac{1}{4}
$$

there is a unique mixed-strategy equilibrium with similar properties as those in case of listing fees, and agglomeration and segmentation occur with positive probability.

The mixed-strategy equilibrium is fully characterized in the proof of Proposition 7 in the Appendix. As is evident from the proposition, the outcome with per-transaction fees resembles the one with listing fees. First, if competition between sellers is weak (that is, the ratio of duopoly to monopoly profit is relatively high), a pure-strategy agglomeration equilibrium results with either type of fees, and platforms compete each other down to fees equal to marginal cost. We note that the conditions for the agglomeration equilibrium to occur coincide in Propositions 1 and 7) with listing fees, the condition is $\pi^{d} / \pi^{m} \geq 1 / 2$, which is the same as that with per-transaction fees, $\pi^{d}(0) / \pi^{m}(0) \geq 1 / 2$. 
Second, if competition between sellers is fierce, a pure-strategy segmentation equilibrium occurs. Here, platforms set the fee equal to $\phi^{m}$ to obtain the largest per-buyer profit - similar to the case of listing fees. The conditions also have a similar interpretation. With listing fees, the condition for the segmentation equilibrium to exist is $\pi^{d} / \pi^{m} \leq 1 / 4$, as a platform should have no incentive to attract both sellers and all buyers in each category instead of only one seller and half of the buyers. With per-transaction fees, the condition is $\left[\phi^{\prime} D^{d}\left(\phi^{\prime}\right)\right] /\left[\phi^{m} D^{m}\left(\phi^{m}\right)\right] \leq 1 / 4$, which rests on the same idea: given the rival's fee $\phi^{m}$, in any category, a platform would attract both sellers and all buyers with a fee of $\phi^{\prime}$. The condition precludes that such a deviation is profitable.

Finally, in the remaining region, a unique mixed-strategy equilibrium exists. As we show in the proof of Proposition 7, this mixed-strategy equilibrium has properties similar to those with listing fees, involving mixing either on a convex or on a non-convex set.

We illustrate the result obtained with Example 1. For $t \geq(v-c) / 2$, the condition $\pi^{d}(0) / \pi^{m}(0) \geq 1 / 2$ is fulfilled, and a pure-strategy agglomeration equilibrium occurs with fees equal to 0 . By contrast, for $t<(v-c) / 4$, the above segmentation equilibrium is the unique equilibrium. In the intermediate range, the mixed-strategy equilibrium occurs. Therefore, mixing occurs for a larger range of parameters than with listing fees - in the latter case, a mixed-strategy equilibrium emerges only for $(v-c) / 3 \leq t<(v-c) / 2$.

\subsubsection{Revenue Sharing}

Another pricing instrument that platforms often use is a percentage fee on the revenue made by sellers. For example, application platforms such as the Appstore or Google Play usually charge a percentage fee of $30 \%$ on the seller's revenue.

Such revenue sharing can also be incorporated into our model. Suppose, again, that the game proceeds as laid out above but that each platform $i=A, B$ extracts a revenue share $r_{i} \in[0,1]$ on each transaction it enables. The seller's profit is then

$$
\pi^{d}\left(r_{i}\right)=\left[\left(1-r_{i}\right) p^{d}\left(r_{i}\right)-c\right] D^{d}\left(r_{i}\right)
$$

in duopoly, and

$$
\pi^{m}\left(r_{i}\right)=\left[\left(1-r_{i}\right) p^{m}\left(r_{i}\right)-c\right] D^{m}\left(p^{m}\left(r_{i}\right)\right)
$$

in monopoly, where $D^{m}\left(r_{i}\right)$ (respectively, $D^{d}\left(r_{i}\right)$ ) is the demand in the seller's monopoly solution (respectively, the sellers' duopoly solution) if platform $i$ demands a revenue share of $r_{i}$. Applying the Implicit Function Theorem, it is easy to show that under standard assumptions on demand, $p^{d}\left(r_{i}\right)$ and $p^{m}\left(r_{i}\right)$ are increasing in $r_{i}$, as long as costs are strictly positive; if $c=0$, prices are independent of $r_{i}$. In addition, both profits and demands are decreasing in $r_{i}$. 
We again impose the reasonable assumption that

$$
\frac{\partial \pi^{m}\left(r_{i}\right)}{\partial r_{i}} \leq \frac{\partial \pi^{d}\left(r_{i}\right)}{\partial r_{i}} \leq 0 \quad \text { and } \quad \frac{\partial D^{m}\left(r_{i}\right)}{\partial r_{i}} \leq \frac{\partial D^{d}\left(r_{i}\right)}{\partial r_{i}} \leq 0
$$

which is fulfilled in the examples given in Section 2. The model with revenue sharing can be analyzed in the same way as the one with per-transaction fees. Although the exact conditions for the boundaries of the equilibrium regions are slightly different, we can show that the results closely resemble those with per-transaction fees ${ }^{31}$ Also, under revenue sharing, platforms do not extract the entire profits of sellers in the segmentation equilibrium: platforms set $r=r^{m}$, with $r^{m}=\arg \max _{r_{i}} r_{i} p^{m}\left(r_{i}\right) D^{m}\left(p^{m}\left(r_{i}\right)\right) / 2$. Thus, platforms obtain the highest profit that is compatible with segmentation in stage 2 . Thus, our main insight carries over to the setting with revenue sharing between platforms and sellers.

\subsubsection{Two-Part Tariffs}

So far, we have considered listing fees and per-transaction fees separately. However, platforms could also charge two-part tariffs that combine the two fees (or combine listing fees and revenue shares). Although we do not present this analysis, the results obtained in the separate study of the two cases suggest that they also hold with two-part tariffs. In particular, if the ratio $\pi^{d} / \pi^{m}$ is small, a segmentation equilibrium will also occur under two-part tariffs, as sellers will avoid competition, and platforms can charge positive fees. Similarly, if an agglomeration equilibrium emerges (i.e., if $\pi^{d} / \pi^{m}$ is sufficiently large), both fees within the two-part tariff are driven down to zero, implying that we obtain the same result as in the analysis of the two separate cases.

In addition, the listing fee avoids distortions of the sellers' product market prices and is, therefore, the more efficient instrument in the two-part tariff. It follows, for example, that in the pure-strategy segmentation equilibrium, both platforms will set a listing fee of $\pi^{m} / 2$ and a per-transaction fee of zero, as this guarantees the highest profit. As a consequence, even if we allowed for two-part tariffs, the results would be similar to those obtained in the model with pure listing fees.

The superiority of the listing fee compared to the transaction fee (or revenue share) follows from the homogeneity of categories and sellers. Suppose, instead, that categories were heterogeneous. This implies that sellers in different categories are also heterogeneous. For example, each category has a different likelihood of being the product category that buyers prefer. Thus, there are more-popular and less-popular categories. Sellers in less-popular categories then obtain a lower expected revenue. As product market prices

\footnotetext{
${ }^{31} \mathrm{We}$ omit the proof of this result as is it follows that of Proposition 7
} 
do not react to listing fees but to transaction fees (and revenue shares), a platform can optimally ensure sellers' participation in less-popular categories with a mixture of both fees. Thus, with heterogeneous product categories, in a segmentation equilibrium, a platform does not necessarily charge only listing fees. In the extreme, as the likelihood that some product categories will be the preferred ones becomes negligible, a platform may not want to rely on listing fees, as this would imply the unavailability of some product categories. Platforms will then exclusively use transaction fees or revenue shares.

\subsection{General Number of Sellers and Platforms}

In this subsection, we extend our model to a finite number of platforms and sellers per category. Suppose that there are $M$ sellers (per category) and $N$ platforms, with $M, N>1$. We denote the per-buyer profit of a seller competing with $m-1$ others sellers by $\pi(m)$, with $\pi(m) \geq \pi(m+1) \geq 0 \forall m=1, \ldots, M-1$-in terms of the notation of the baseline model, $\pi(1)=\pi^{m}$ and $\pi(2)=\pi^{d}$. All other assumptions and the equilibrium refinement in the second stage are the same as in main model.

In addition, we also impose an equilibrium selection criterion in the first stage. With a general number of platforms and sellers, the equilibrium in the fee-setting game between platforms may not be unique. Then, as a refinement, we assume that platforms choose the profit-dominant equilibrium.

The main differences from our baseline model are twofold: First, with a general number of sellers and platforms, the number of sellers is no longer necessarily a multiple of the number of platforms. The question is, therefore, how sellers, in order to make buyers indifferent, allocate if multiple platforms carry a positive volume of trade. Second, it may be optimal for platforms in the first stage to exclude sellers via the choice of their listing fees. As we will demonstrate below, this may occur in a segmentation equilibrium.

Following the same structure as with different pricing instruments, we characterize in the next proposition the regions in which the different types of equilibria exist, thereby pointing out the analogy to the simpler baseline model. To write the proposition in the most concise form, we define $k$ as the largest integer, such that $M \geq k N$. For example, if $M=11$ and $N=4$, then $k=2$.

Proposition 8. Consider the case in which $M \geq N$ :

If

$$
\frac{\pi(k+1)}{\pi(k)} \geq \frac{1}{N}
$$

in equilibrium $f_{i}^{\star}=0, \forall i=1, \ldots, N$, platform profits are 0 , and there is positive trade only on a subset of platforms. 
If for some $l \in\{1, \ldots, k\}$

$$
\frac{\hat{m} \pi(\hat{m})}{l \pi(l)} \leq \frac{1}{N}
$$

with $\hat{m} \in \arg \max _{l<m \leq M} m \pi(m)$, in the unique profit-dominant equilibrium $f_{i}^{\star}=\pi\left(l^{\star}\right) / N$, with $l^{\star} \in \arg \max _{l} l \pi(l)$ for all $l \in\{1, \ldots, k\}$ that satisfy (2), platform profits are $\Pi_{i}^{\star}=$ $l^{\star} \pi\left(l^{\star}\right) / N \forall i=1, \ldots, N$, and all platforms carry a positive volume of trade.

If neither (1) nor (2) is satisfied, there is a unique profit-dominant mixed-strategy equilibrium, in which platforms make positive profits.

Consider the case in which $M<N$ : In equilibrium, $f_{i}^{\star}=0 \forall i=1, \ldots, N$, and platform profits are 0.

If there are at least as many sellers (in each category) as platforms, the proposition demonstrates that the qualitative features of the equilibrium are similar to those in the baseline model. If competition between sellers is relatively moderate, a seller's profit when one additional seller joins the platform falls only by a small amount (i.e., $\pi(k+1) / \pi(k)$ is relatively large), which implies that condition (1) is satisfied. Thus, equilibrium platform fees are zero.

In analogy to the baseline model, this equilibrium prevails if sellers prefer to be active only on a subset of platforms, given that all platforms charge zero fees. To relate this to condition (1), note that $k$ is the largest number of sellers, so that all platforms have positive trade volume, and each one hosts $k$ sellers (so that buyers are willing to split between platforms). Condition (1) states that such a configuration will not emerge in the second stage, as sellers have an incentive to deviate ${ }^{32}$ With trade occurring only on a subset of platforms, no platform can charge a strictly positive fee as it loses its buyers and sellers to a competitor with zero fee.

In contrast to the baseline model, such an equilibrium does not necessarily lead to full agglomeration, as it may be optimal for some sellers to locate on one platform and other sellers on another. Nevertheless, only a subset of platforms carry a positive volume of trade, which implies at least partial agglomeration, and equilibrium fees of zero. This must also be the equilibrium outcome if the number of platforms exceeds the number of sellers in a category, as it implies that at least one platform will not have a positive volume of trade.

By contrast, in a pure-strategy segmentation equilibrium, all platforms carry a positive volume of trade. In analogy to the baseline model, this equilibrium occurs if competition between sellers is intense. From condition (2), the equilibrium exists if, in each category, every platform hosts $l$ sellers, and no platform can obtain a higher profit by attracting a larger number of sellers (where attracting a number $\hat{m}$ is the most profitable

\footnotetext{
${ }^{32}$ In the baseline model, we have $k=1$, and, thus, condition 1 is equivalent to $\pi^{d} / \pi^{m} \geq 1 / 2$.
} 
one among these deviations). The condition for a segmentation equilibrium to exist in the model with a general number of platforms and sellers resembles that of the baseline model. In the baseline model, we have $l=1$ and $\hat{m}=2$, and, thus, condition (2) is equivalent to $\pi^{d} / \pi^{m} \leq 1 / 4$.

The key difference from the baseline model is that the segmentation equilibrium may lead to the exclusion of some sellers - that is, the equilibrium number of sellers on a platform, $l^{\star}$, may be less than $k$. If $M>k N$, this must be the case, as a segmentation equilibrium involves at least $M-k N$ inactive sellers. However, even if $M=k N$, it can be optimal for platforms to charge such a high fee that some sellers prefer to stay inactive. The reason is that becoming active increases competition and, therefore, would not allow the seller to recover the fee. In addition, with a general number of sellers, a segmentation equilibrium may involve more than one seller in each category on a platform if this allows platforms to obtain a higher profit. As in the baseline model, platforms extract the entire profit from all active sellers.

Finally, in the region in which neither condition (1) nor condition (2) holds, a mixedstrategy equilibrium occurs. The intuition and the properties are the same as in the baseline model.

In our analysis, we consider the situation with a given number of sellers $M$ with positive profit (gross of the listing fee). Instead, if sellers incurred a fixed entry cost $F$, $\pi(m)-F$ would become negative for $m$ sufficiently large, as more intense competition drives down margins. Then, even if platforms charge zero fees, no platform would be host to an unlimited number of sellers. Yet, considering a game with free entry yields similar results to those of Proposition 8. The conditions for the equilibrium regions differ, but the qualitative results that buyers and sellers may segment and that platforms obtain positive profits continue to hold. If there is a finite $m^{\prime}$ as the solution to $\pi\left(m^{\prime}\right) / N-F>0$ and $\pi\left(m^{\prime}+1\right) / N-F<0$, the region in which the agglomeration equilibrium exists shrinks and eventually vanishes as the number of available sellers $M$ becomes sufficiently large. The reason for this result is that with zero fees, each platform would host $m^{\prime}$ sellers. A seller's profit is then strictly positive, which gives each platform an incentive to increase its fee. To sum up, with entry, pure agglomeration cannot occur when the number of sellers that may enter is large and, thus, segmentation becomes more likely.

\subsection{Buyer-Preferred Equilibrium}

In this subsection, we demonstrate how our equilibrium would change if we used the concept of payoff-dominance of buyers (instead of sellers) in the second stage, in addition to coalition-proofness. Because $V^{d}>V^{m}$, buyers prefer an agglomeration equilibrium over a segmentation or stand-alone equilibrium. This implies that whenever the refinement 
of coalition-proofness alone does not suffice to select a unique equilibrium, it is payoffdominant for buyers to choose an agglomeration equilibrium whenever it exists - as above, the two refinements are never in conflict with each other.

Figure 6 shows the different equilibrium regions with this selection rule. As long as at least one platform sets a fee below $\pi^{d}$, an agglomeration equilibrium exists and will be selected. However, if both fees are larger than $\pi^{d}$, an agglomeration equilibrium does not exist, as sellers would obtain negative profits. The selected equilibrium is then the same as in Section 3 because buyers are indifferent between the segmentation and a stand-alone equilibrium: if both exist, only the segmentation equilibrium is coalition-proof.

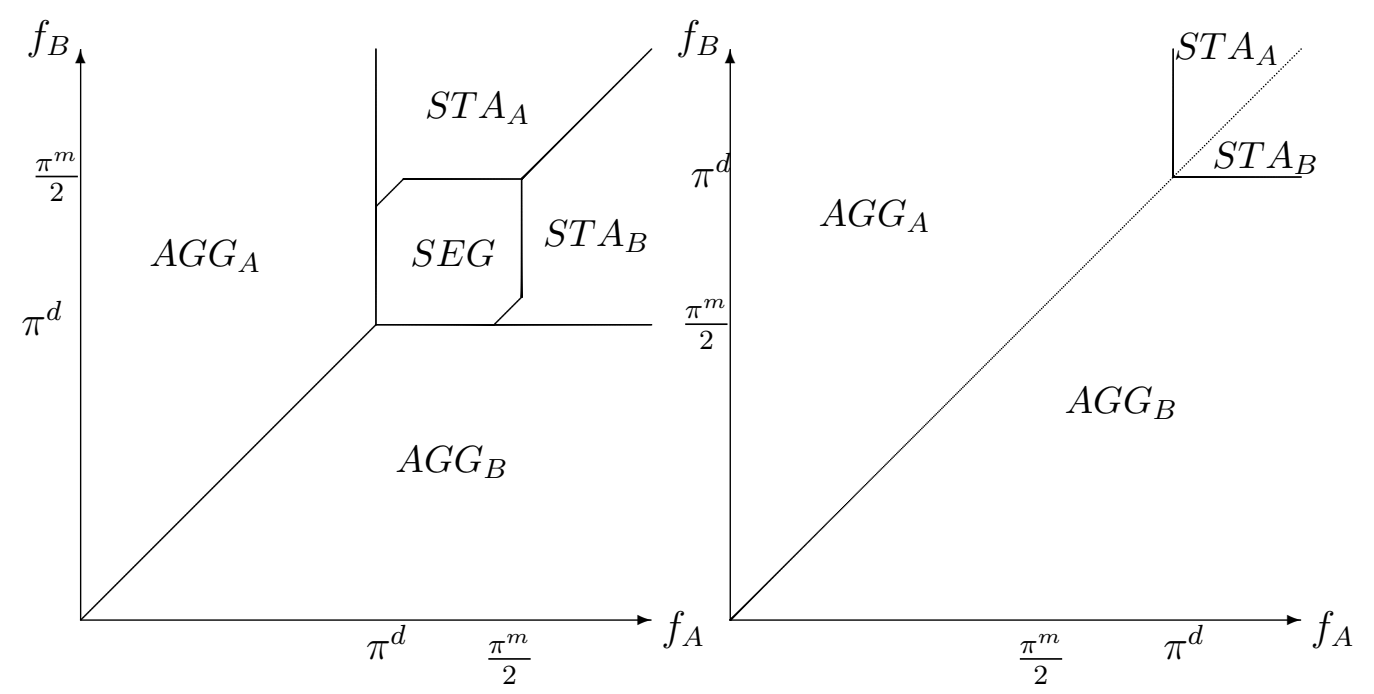

Figure 6: Selected equilibrium configurations with payoff-dominance of buyers

Turning to the first stage, there is now always an equilibrium in which platform fees are equal to 0 . Given that platform $i$ sets $f_{i}=0$, an agglomeration equilibrium exists and will be selected. Hence, platform $-i$ cannot do better than to also set $f_{-i}=0$. However, this equilibrium is not the only one if $\pi^{d} / \pi^{m} \leq 1 / 4$. By the same logic as in Section 3 , if each platform charges a fee of $\pi^{m} / 2$, no platform has a profitable deviation. Therefore, a segmentation equilibrium in which platforms extract the entire profits from sellers also exists and is profit-dominant for platforms. Invoking profit-dominance, the same segmentation equilibrium as in Section 3 emerges. Therefore, our result does not hinge on the selection criterion in stage 2 .

If a buyer-preferred equilibrium is selected, no mixed-strategy equilibrium exists. The reason is as follows: If $\pi^{d} / \pi^{m}>1 / 4$, a platform has an incentive to deviate from the equilibrium candidate $f_{A}=f_{B}=\pi^{m} / 2$ and to set a fee below $\pi^{d}$ to induce agglomeration. The best response of the rival platform is then to undercut this fee slightly, as it cannot induce segmentation with a higher fee (in contrast to the case with a seller-preferred 
equilibrium in stage 2). Then, the standard Bertrand argument applies, leading to zero fees in equilibrium. Therefore, if the buyer-preferred equilibrium is chosen, we obtain $f_{A}^{\star}=f_{B}^{\star}=0$ if $\pi^{d} / \pi^{m}>/ 4$ and $f_{A}^{\star}=f_{B}^{\star}=\pi^{m} / 2$ if $\pi^{d} / \pi^{m} \leq 1 / 4$.

\subsection{Two-Sided Pricing}

In the baseline model, we considered the situation in which platforms can set fees only to sellers. This is a common practice among most trading platforms. A main reason is that buyers are often uncertain about whether or not they want to buy a product, and they first inform themselves on the platform about available offers and product characteristics. Thus, charging a subscription fee will deter many buyers. In addition, some buyers often obtain only a small surplus, and so platforms can charge only a very small fee to keep these buyers on board. With small transaction costs from each payment (e.g., due to fraud), it is more effective to charge sellers who are usually fewer in numbers.

Apart from these justifications for not charging buyers, which are outside the model, we can demonstrate that the segmentation equilibrium derived in the baseline model is robust to two-sided pricing (that is, platforms set a subscription fee to buyers, $f_{b}$, on top of the listing fee to sellers, $f_{s}$ ), provided that negative fees are not possible ${ }^{33}$

We focus on the situation $\pi^{d} / \pi^{m} \leq 1 / 4$, in which the pure-strategy segmentation equilibrium exists with one-sided pricing. Platforms then set a seller fee $f_{s}=\pi^{m} / 2$ and extract the full seller surplus. With two-sided pricing, a fee combination of $\left\{f_{s}=\pi^{m} / 2, f_{b}=0\right\}$ for both platforms is no longer an equilibrium under the refinement of coalition-proofness and seller-dominant equilibrium. To see this, suppose that platform $-i$ sets $\left\{f_{s}=\right.$ $\left.\pi^{m} / 2, f_{b}=0\right\}$. Platform $i$ can then set fees equal to $\left\{f_{s}=\pi^{d}-\epsilon, f_{b}=V^{d}-V^{m}\right\}$ and attract all sellers and buyers because sellers obtain a profit of $\epsilon>0$ on platform $i$ instead of 0 on platform $-i$. Therefore, the coalition of all sellers and buyers on platform $-i$ is better off by moving to platform $i$, as buyers are indifferent and obtain a payoff of $V^{m}$ on both platforms (it can be shown that setting fees equal to $\left\{f_{S}=\pi^{d}-\epsilon, f_{B}=V^{d}-V^{m}\right\}$ is, indeed, the most profitable coalition-proof deviation). The profit of platform $i$ is then (almost) equal to $2 \pi^{d}+V^{d}-V^{m}$. Although we are in the region with $\pi^{d} / \pi^{m} \leq 1 / 4$, the profit from deviating is larger than $\pi^{m} / 2$, as $4 \pi^{d}+2\left(V^{d}-V^{m}\right)>\pi^{m}$ due to the fact $V^{d}+\pi^{d}>V^{m}+\pi^{m}$.

\footnotetext{
${ }^{33}$ With negative fees, a divide-and-conquer strategy can destabilize the segmentation equilibrium. Under divide-and-conquer, a deviating platform sets a sufficiently low fee on one side to ensure that this side participates for sure. It can then use the fee on the other side to extract surplus on that side. In particular, a platform deviating from the segmentation equilibrium can attract sellers with negative fees and extract the full surplus generated on the buyer side. However, such negative fees are usually not feasible, as they generate losses for platforms from otherwise uninterested participants who inflate participation levels without generating any transaction opportunities.
} 
Determining the equilibrium for the range $\pi^{d} / \pi^{m} \leq 1 / 4$, we obtain (following arguments similar to those in the baseline model) that the unique equilibrium under our

refinement is $\left\{f_{s}^{\star}=0, f_{b}^{\star}=V^{m}\right\}$ and segmentation occurs. With these fees, no platform can attract more sellers since this would lead to competition between them and, therefore, to a reduction in sellers' profits. Instead of extracting the sellers' profits, platforms do not leave surplus to buyers. Importantly, though, despite this difference in fees between onesided and two-sided pricing, the main intuition for the segmentation equilibrium to occur is the same: sellers avoid competition by being active on both platforms, and platforms exploit this role of segmenting the market by charging strictly positive fees.

If in stage 2 buyers and sellers play the equilibrium that buyers prefer (in addition to coalition-proofness), the equilibrium fees would be the same as in the case of one-sided pricing - that is, $\left\{f_{s}^{\star}=\pi^{m} / 2, f_{b}^{\star}=0\right\}$. Setting a strictly positive fee to buyers can never be profitable for a platform, as then all buyers prefer the rival platform. Given this, the same arguments as in Section 5.3 apply. Although buyers prefer agglomeration, platforms avoid this in equilibrium by setting listing fees to sellers above $\pi^{d}$.

\subsection{Further extensions}

In our preceding analysis, we have shown the robustness of the tradeoffs involved between segmentation and agglomeration in a number of settings. However, we maintained the independence between categories and the homogeneity of buyer behavior. In this section, we discuss what happens in richer settings that allow for these features.

\subsubsection{Interdependence between Categories}

\section{Competition across categories}

In our analysis, we assumed that each buyer is interested in exactly one product category. Suppose, instead, that a buyer receives a positive gross utility from buying a product in a category other that her preferred one (which, by construction, is less than from products in her preferred category). Then, products in different categories are substitutes. As a consequence, the demand for a product in the preferred category may be lower if prices in different categories are lower. Sellers will take this into account in their pricing decisions, implying that products from different categories may impose competitive constraints. As a consequence, prices will be weakly lower than in our model. This leads to lower values of $\pi^{m}$ and, possibly, of $\pi^{d}$. Hence, in the segmentation equilibrium, platforms' fees will be weakly lower, and, depending on the profit ratio $\pi^{d} / \pi^{m}$, the regions for the segmentation and agglomeration equilibrium will be affected by competition across platforms. However, the main effects driving the results are still present, and our main insights are robust. 
We can restate our main conclusions in a different setting, in which there is only one product (and, thus, one seller) per product category. With this simplification, since sellers do not compete, the market necessarily features agglomeration. Introducing competition between categories, the equilibrium switches to segmentation if segmentation sufficiently reduces seller competition. In such a segmentation equilibrium, half of the categories are listed on platform $A$ and the other half on platform $B$. This result is in the same spirit as our findings in the baseline model.

\section{Platforms with diseconomies}

Another source of interdependence between categories could be platform diseconomies in the number of categories. Platforms experience such diseconomies if the number of product categories on a platform has a direct and negative effect on buyer utility. Such congestion externalities can be present because, for instance, it becomes more costly for a buyer to find the preferred product category as the number of categories increases. This implies that a buyer experiences a utility loss if the number of listed categories is large. If platforms can endogenously choose this number, then, to avoid repelling buyers, they may not list some categories in equilibrium. This implies that even in an agglomeration equilibrium, listing fees may be positive, as lowering the fee to zero induces the participation of sellers in all categories, which is not attractive to buyers 34

Alternatively, the number of product categories may affect buyer utility indirectly. This happens if the optimal presentation of products on a platform depends on the product category. Suppose that a platform has to commit to a unique format for presenting products (e.g., to avoid confusing buyers). Then, if very different product categories are listed, the presentation format is not optimal for some products, and, thus, the utility of buyers who prefer these products is reduced. As above, a consequence is that some products will be delisted 35

\subsubsection{Heterogeneous Buyer Behavior}

In our model, buyers are ex ante identical. In particular, they do not prefer one platform over the other. Although this assumption ensures that platforms are fully homogeneous ex ante and, therefore, strengthens our theoretical contribution, it may not be in line with the consumer behavior observed in some markets. For example, a fraction of buyers may be loyal to a platform. Similarly, some buyers may decide very quickly on which platform

\footnotetext{
${ }^{34}$ One could also imagine that product categories differ by the probability of being the preferred category. Then, popular categories will be listed, but less popular categories will be delisted.

${ }^{35}$ An example in which a platform did not cater well to buyer tastes with its presentation of particular product categories is the market for handmade and vintage items on Ebay. Newer platforms, such as Etsy and Dawanda, offered sellers the opportunity to offer more information, and this led to a quick migration of buyers and sellers to these new platforms. This suggests that Ebay was subject to diseconomies in the number of listed product categories and lost out to newcomers.
} 
to be active and, therefore, assign themselves randomly to a platform. Suppose that each platform has a fraction $\beta / 2$ of loyal buyers and that all other buyers are shoppers-i.e., they join the platform that offers the highest expected utility as in our baseline model. To reach loyal buyers, sellers have to list on both platforms (as, for example, in hotel booking or food delivery services). Thus, if sellers can multi-home, in an equilibrium that corresponds to an agglomeration equilibrium, both platforms set a positive listing fee equal to the duopoly profit that a seller earns from loyal buyers so as to induce seller multi-homing. By contrast, in an equilibrium that corresponds to a segmentation equilibrium-i.e., each platform attracts half of the shoppers - sellers single-home along the equilibrium path because the insights from Section 4.2 still hold.

\section{$6 \quad$ Policy Implications and Predictions}

\subsection{Policy Implications}

Our paper has several normative implications that can guide policy makers in regulating platform markets. These implications rest on the insight that market segmentation has undesirable welfare properties: segmentation leads lower total welfare and consumer welfare than agglomeration due to less choice for buyers and higher product prices. However, platforms benefit from segmenting the market, as this allows them to extract rents from sellers. This conflict between what is in the interest of platforms and what is in the interest of society may justify policy intervention.

It is important for policy makers to distinguish between segmentation due to multiple active platforms enabling sellers to reduce competition, and segmentation resulting from inherent differentiation between platforms. In the former case, as just pointed out, segmentation is welfare-decreasing, whereas in the latter case, platforms cater to different buyer (and perhaps seller) tastes, which, all else equal, is welfare-increasing. It seems possible to find out the cause of segmentation, as the degree of product differentiation can be measured by appropriate tests (e.g., SNIPP test). ${ }^{36}$

Beyond this general observation, our framework generates a number of specific implications for antitrust, regulation, and merger control.

Exclusive contracts. A widely-discussed issue in platform markets is the use of exclusive contracts ${ }^{37}$ Such contracts restrict sellers to offering their products exclusively on one platform (in exchange for a favorable deal on the fee charged by the platform).

\footnotetext{
${ }^{36}$ See Affeldt, Filistrucchi and Klein (2013) for an application of the SNIPP test to platform markets.

${ }^{37}$ For example, in the video game industry, console platforms often impose console exclusivity, which prevents game developers from selling a similar game on rival consoles (see Lee, 2013, for an in-depth analysis). As another example, trading platforms sometimes require 'special' offers by sellers to be exclusive on them.
} 
Therefore, these exclusive contracts rule out seller multi-homing. As we showed in Section 4.2, if sellers can multi-home, an agglomeration equilibrium is more likely to arise, implying that a restriction to single-homing is anti-competitive. In this respect, our paper provides a new rationale for why exclusive contracts are welfare-decreasing: they help sellers to commit to a single platform, thereby sustaining market segmentation.

Price caps on listing fees. A policy instrument to curb firms' market power in general is to set price caps. At first glance, this might also look attractive in our framework as a way to tame the market power of platforms vis-a-vis sellers (i.e., platforms charge strictly positive listing fees only in a segmentation equilibrium). Therefore, one may think that regulating fees via caps can lead to agglomeration. However, the fundamental problem is that even with low fees, sellers choose segmentation by themselves if they obtain higher profits with this configuration. Therefore, caps on listing fees are not helpful to achieve agglomeration and are merely a rent-shifting device.

Merger control. Suppose that sellers in some product categories consider merging ${ }^{38}$ Such mergers generally reduce competition in the particular product category and, as a consequence, favor agglomeration. Thus, our theory predicts that market tipping is more likely in industries following mergers between sellers. If there is segmentation prior to the merger, but agglomeration afterwards, a merger may increase total welfare, and even consumer welfare, as buyers find, in expectation, a better match. On the downside, they pay the prices set by a two-product instead of a single-product monopolist, which are higher if products are substitutes. Overall, we have identified an efficiency defense for mergers among sellers in a platform market: if the merging sellers operate on different platforms prior to the merger, the merger may make the market tip, which can be beneficial to buyers, as it improves the match quality ${ }^{39}$

\subsection{Predictions}

Our theory leads to novel predictions that are empirically testable.

Correlation between market concentration and fee levels in the platform market. Our theory predicts a negative correlation between market concentration and the level of the listing fee in platform markets. More precisely, for a given number of available platforms, our theory predicts that the relation between market concentration, in terms of market share, and the level of the (average) listing fee is negative. In markets that feature agglomeration, platform fees are lower than in markets in which platforms have a more

\footnotetext{
${ }^{38}$ Such mergers occurred in several intermediated industries in recent years. For example, in the housing market, big real estate agencies acquired many smaller companies. Similarly, in the video game industry, several software-developing companies have recently merged.

${ }^{39}$ If, prior to the merger, the market is characterized by agglomeration, the merger leads to multiproduct monopoly instead of duopoly prices. Thus, it necessarily decreases consumer welfare.
} 
equal market share. This reverses the prediction of standard theory. Our prediction is testable, for example, by analyzing cross-industry or cross-country variation in the data. To make a meaningful comparison, one would need to condition on the market characteristics and then consider the correlation between the Hirshman-Herfindahl Index (HHI) of active firms and the level of the listing fee. There would be support for our theory if platform fees were larger in markets with a smaller HHI.

Correlation between market characteristics in the product market and concentration in the platform market. Our theory provides a prediction concerning the relation between the prevailing conditions in the intermediated product market and the market outcome in the platform market. Specifically, if competition in the intermediated product market is weak, the platform market will be more concentrated (measured by the HHI). Possible sources for different degrees of competitiveness in the product market are manifold. They could be due to differences in the degree of product differentiation or differences of the ratio between sellers and buyers in thin markets. The prediction can be tested, for example, by looking at different regional markets within the same industry and country or by considering different broadly defined product categories. As platforms usually operate country-wide and across broad product categories, the same number of platforms may well be present, but concentration may vary between different regions or product categories. If a product market has characteristics that are unfavorable to strong competition between sellers - i.e., profits are relatively high even if sellers compete- our theory predicts agglomeration. Thus, reduced competition among sellers tends to lead to market tipping.

As an example, consider price comparison websites. Suppose that buyers consider a purchase within a broad product category and do not yet know which specific product they like. In broad product categories in which there is little room for differentiation between sellers' offers, competition between sellers is intense, and, thus, it is likely that sellers segment. The opposite holds in the broad product categories in which product differentiation between sellers is pronounced.

\section{Conclusion}

In this paper, we have proposed a theory of competing platforms that enable trade between buyers and sellers. Platforms are homogeneous and charge fees to sellers, and sellers compete in the product market. We have analyzed how the competitive conditions in the seller market affect platform market structure.

Can multiple platforms exist and earn positive profits even if there is no differentiation between them? We show that the function of multiple platforms as an endogenous 
segmentation device for competing sellers can explain such an outcome. Sellers choose to be active on different platforms to avoid fierce competition. Platforms exploit this by setting positive fees, and obtain strictly positive profits. Thus, multiple homogeneous platforms have a positive market share. Such a segmentation equilibrium exists if competition between sellers is sufficiently strong. If, by contrast, there is little competition between sellers, the standard intuition is confirmed: the equilibrium features agglomeration, and platform fees are low. As a consequence of these results, the relation between market concentration and fees is negative. Platform fees are low in concentrated markets but high if market shares are similar.

Our main insights are robust to several different model formulations - i.e., they do not depend on the platform pricing instrument, the possibility of buyer and seller multihoming, and the number of platforms and sellers. In addition, the framework generates several policy implications and predictions that are empirically testable.

In our model, we did not consider endogenous entry of platforms. Although a dominant platform emerges in our agglomeration equilibrium, fees are driven down to marginal costs, and the market is contestable with the presence of competitors. By contrast, if platforms incurred fixed costs from entry, only one platform would enter whenever the equilibrium is characterized by agglomeration. This single platform would then act as a monopolist and charge a high fee. While such monopoly positions may occur, in reality, we observe that in several platform markets, there is a dominant platform together with some competitors who only have negligible market shares. These competing platforms may, however, have larger market shares in related markets and incur little cost to serve buyers and sellers also in markets in which they are currently negligible. For example, in the second-hand good market, Ebay can be considered as a dominant platform for many broadly defined product segments. However, Ebay's market power is restrained by other platforms, small (e.g., Ebid and Bonanza) or large (Amazon), elsewhere ${ }^{40}$

We placed our analysis in a static context and focused on platform pricing and subsequent subscription decisions of buyers and sellers. We leave extensions such as dynamic competition between platforms and the platforms' innovation incentives for future research.

\footnotetext{
${ }^{40}$ In some product segments, the dominant platform may alternatively be a specialized one with a smaller selection of products. A case in point is Etsy, which tends to specialize in handcrafted products and may be considered dominant in the United States. While Ebay essentially lost this market segment to Etsy, it remains a potential competitor.
} 


\section{Appendix A: Equilibrium Selection in Stage 2}

In the first step, we determine all Nash equilibria in stage 2 , given fees $\left(f_{i}, f_{-i}\right)$. In a second step, we show how the refinement of coalition-proofness plus profit dominance of sellers singles out an essentially unique equilibrium. The qualification to the uniqueness is that if $f_{i}=f_{-i}$, under some conditions two payoff-equivalent equilibria exist. As will become evident below, these equilibria are agglomeration either on platform $A$ or $B$, or stand-alone either on platform $A$ or $B$, dependent on the level of $f_{i}$. However, as the equilibria are payoff-equivalent, selecting among them is not necessary.

Suppose that the mass of sellers is unequal on both platforms, i.e., $\alpha_{i}$ sellers are on platform $i$ and $\alpha_{-i}<\alpha_{i}$ sellers are on platform $-i$. Then all buyers will join platform $i$. This implies that sellers on platform $-i$ have a profitable deviation to either go the platform $i$ or be inactive for any $f_{-i}>0$. It follows that in equilibrium either one platform has no sellers and no buyers, or $\alpha_{i}=\alpha_{-i}$, which makes buyers indifferent and induces them to split equally between the two platforms under our tie-breaking rule.

We start with the situation, in which there is trade on only one platform. As sellers are homogeneous across categories, there cannot be an equilibrium in which sellers in different categories follow different strategies. The reason is that if it is profitable for one or both sellers in some categories to list on the platform with a positive volume of trade, this must also be true for sellers in the remaining categories. There can be two equilibrium configurations in which only one platform carries a positive volume of trade.

The first configuration is an agglomeration equilibrium, in which all sellers and all buyers agglomerate on one platform. A seller's profit is then $\pi^{d}$. Hence, an equilibrium with agglomeration on platform $i$ exists if $f_{i} \leq \pi^{d}$, independent of $f_{-i}$. The second equilibrium configuration is a stand-alone equilibrium, in which in each category only one seller is active on platform $i$ and all buyers go to platform $i$. This configuration occurs if $\pi^{m} \geq f_{i}>\pi^{d}$, independent of $f_{-i}$. This equilibrium cannot occur with $f_{i}<\pi^{d}$, as in this case both sellers in each category prefer to be active on platform $i$.

We now turn to the equilibrium configuration, in which $\alpha_{i}=\alpha_{-i}$. The following three types of seller compositions give rise to $\alpha_{i}=\alpha_{-i}$.

(i) In each category, one seller lists on platform $i$ and one seller lists on platform $-i$.

(ii) In $1 / 2$ of the categories, both sellers list on platform $i$ and in the other half both sellers list on platform $-i$.

(iii) In $1 / 2$ of the categories, one seller lists on platform $i$ and in the other half one seller lists on platform $-i$.

In addition, any convex combination of these three seller compositions (i.e., mixing between the three types) leads to $\alpha_{i}=\alpha_{-i}$. Note that it can never be an equilibrium that 
in fewer than $1 / 2$ of the categories both or one seller list on platform $i$ and platform $-i$. The reason is that non-active sellers have a profitable deviation to become active. This is because platform fees must be such that the resulting profits are higher than the listing fees as otherwise there can be no categories in which sellers are willing to list.

We now show that types (ii) and (iii) can never occur in equilibrium. Consider case (ii). Since in $1 / 2$ of the categories, both sellers are active on platform $i$, we must have $f_{i} \leq \pi^{d} / 2$. If a seller active on platform $-i$ then deviates to platform $i$, its profit changes from $\pi^{d} / 2-f_{-i}$ to $\pi^{m} / 2-f_{i}$. By a similar argument, if a seller deviates from platform $i$ to platform $-i$, its profit changes from $\pi^{d} / 2-f_{i}$ to $\pi^{m} / 2-f_{-i}$. This implies that case (ii) can only be an equilibrium if $\pi^{d} / 2-f_{-i} \geq \pi^{m} / 2-f_{i}$ and $\pi^{d} / 2-f_{i} \geq \pi^{m} / 2-f_{-i}$. Since $\pi^{d} / \pi^{m}<1$, both conditions cannot jointly hold, implying that there must be a profitable deviation. Similarly, in case (iii) platform fees must be smaller than $\pi^{m} / 2$, which implies that non-active sellers have a profitable deviation to list on the platform in which the competitor is not active. Since those two types cannot be an equilibrium configuration, mixing among the three types can also be excluded by the same arguments.

As a consequence, the configuration in which both platforms are active can only be such that each platform is host to one seller in each category. This equilibrium can only occur if platform fees are below $\pi^{m} / 2$, and no seller has an incentive to deviate and become active on the other platform. The latter condition implies

$$
\frac{\pi^{m}}{2}-f_{i} \geq \frac{\pi^{d}}{2}-f_{-i}
$$

Rewriting this condition, we obtain that a segmentation equilibrium exists if and only if

$$
f_{i} \leq \min \left\{\frac{\pi^{m}-\pi^{d}}{2}+f_{-i}, \frac{\pi^{m}}{2}\right\}
$$

As illustrated in Figure 1, for any combination of listing fees $\left(f_{i}, f_{-i}\right)$ with $f_{i} \leq \pi^{m}$ and $f_{-i} \leq \pi^{m}$, multiple equilibria exist in stage 2 .

Finally, we note that for all $\left(f_{i}, f_{-i}\right)$ a no-trade Nash equilibrium exists in which neither buyers nor sellers participate on either platform. However, this no-trade equilibrium is not coalition-proof whenever some other equilibrium exists. We therefore disregard it in the following discussion.

In the second step, we demonstrate how our selection rule singles out a unique equilibrium (unique subject to the qualification above). We start with the cases in which only a single equilibrium configuration exists (i.e., agglomeration or stand-alone) but multiple equilibria occur because agents can coordinate on either platform. First, consider the case in which there are the two equilibria, where all sellers (and buyers) agglomerate on platform $A$ or platform $B$. If both platforms charge a fee below $\pi^{d}$ and $f_{i} \neq f_{-i}$, coalition- 
proofness implies that all sellers and buyers coordinate on the platform with the lower fee, that is, they agglomerate on platform $i$ if $f_{i} \leq f_{-i} \stackrel{41}{4}$ Within this coalition, there is also no subcoalition that can improve by being active on platform $-i$. Similarly, if both platforms charge a fee larger than $\pi^{d}$ and the two equilibria in which only half of the sellers are active on platform $A$ or on platform $B$ exist (i.e., the stand-alone equilibria), then sellers choose platform $i$ if and only if $f_{i}<f_{-i}$.

Now we turn to cases, in which multiple equilibrium configurations exist. First, consider the case in which agglomeration and stand-alone equilibria exist. From the arguments above, this occurs if one platform, say platform $-i$, charges a fee below $\pi^{d}$ whereas the other one charges a fee above $\pi^{d}$. However, the stand-alone equilibrium is then not coalition-proof because a coalition consisting of all buyers and all inactive sellers has a profitable deviation. If all these agents choose to be active on platform $-i$, then buyers are indifferent (as the same number of sellers is then active on each platform) but the profits of the formerly inactive sellers strictly increase from 0 to $\pi^{m}-f_{i}>0$. In addition, no subcoalition can deviate and be strictly better off. By the same argument, if stand-alone and segmentation equilibria exist, a stand-alone equilibrium is not coalitionproof, whereas a segmentation equilibrium is. To see this, note that for these equilibrium configurations to co-exist, we must have that $\pi^{d} / \pi^{m}<1 / 2$ and that both fees are between $\pi^{d}$ and $\pi^{m} / 2$. Thus, no coalition of sellers has the incentive to deviate from a segmentation equilibrium 42

Finally, we turn to the region, in which segmentation and agglomeration equilibria exist. The profit of each seller in an agglomeration equilibrium on platform $i$ is $\pi^{d}-f_{i}$. By contrast, in a segmentation equilibrium, the profit of a sellers is either $\pi^{m} / 2-f_{i}$ or $\pi^{m} / 2-f_{-i}$ dependent on which platform the seller is active. Let us first look at the case $\pi^{d} / \pi^{m} \geq 1 / 2$. It is evident that a coalition of all sellers active on the platform with the higher fee, say platform $i$ (i.e., $f_{i} \geq f_{-i}$ ), and all buyers on this platform have a profitable deviation to switch to platform $-i$. After such a deviation, the sellers are (weakly) better off because $\pi^{d}-f_{-i} \geq \pi^{m} / 2-f_{i}$ due to the fact that $\pi^{d} / \pi^{m} \geq 1 / 2$ and $f_{-i} \leq f_{i}$, and buyers are better off because they observe the offers of both sellers and not only one. It follows that for $\pi^{d} / \pi^{m} \geq 1 / 2$, the segmentation equilibrium is eliminated ${ }^{43}$

We now turn to the case $\pi^{d} / \pi^{m}<1 / 2$. We first show that a similar mechanism as the one in the previous paragraph does only partly work then. In particular, sellers on platform $i$ (i.e., the platform with the higher fee) prefer agglomeration on platform $-i$

\footnotetext{
${ }^{41}$ This is also the profit-dominant equilibrium for sellers.

${ }^{42}$ In these regions, profit-dominance of sellers either selects the same equilibrium as coalition-proofness, or profit-dominance has no bite as some sellers prefer stand-alone over agglomeration (or segmentation) whereas others have the opposite preference. Hence, coalition-proofness and profit-dominance are not in conflict with each other.

${ }^{43}$ Again, profit-dominance of sellers also eliminates the segmentation equilibrium.
} 
over segmentation if and only if $\pi^{d}-f_{-i} \geq \pi^{m} / 2-f_{i}$ or

$$
f_{i} \geq \frac{\pi^{m}}{2}-\pi^{d}+f_{-i}
$$

If this inequality holds, the segmentation equilibrium is not coalition-proof because sellers and buyers on platform $i$ can profitably deviate and agglomerate on platform $-i$. This shrinks the range for the segmentation equilibrium. In particular, for fees below $\pi^{d}$, the equilibrium exists for $f_{i} \leq\left(\pi^{m}-\pi^{d}\right) / 2+f_{-i}$, whereas it survives the refinement only if $f_{i}<\pi^{m} / 2-\pi^{d}+f_{-i}{ }^{44}$ If $f_{i}<\pi^{m} / 2-\pi^{d}+f_{-i}$, the refinement of coalition-proofness has no bite. However, the refinement of profit-dominance for sellers then selects the segmentation equilibrium as the unique equilibrium. In particular, the inequality ensures that sellers on platform $i$ are better off in the segmentation equilibrium than in the agglomeration equilibrium, and the condition $\pi^{d} / \pi^{m}<1 / 2$ guarantees that also sellers on platform $-i$ prefer segmentation over agglomeration because $\pi^{m} / 2-f_{-i}>\pi^{d}-f_{-i}$.

Therefore, our equilibrium refinement selects the following equilibrium, given any $\left(f_{i}, f_{-i}\right)$ with $f_{i} \leq \pi^{m}$ and $f_{-i} \leq \pi^{m}$ :

(i) If $\pi^{d} / \pi^{m} \geq 1 / 2$, then

- for $f_{i}, f_{-i} \geq \pi^{d}$, the equilibrium is $S T A_{i}$ if $f_{i} \leq f_{-i}$;

- for all other values, the equilibrium is $A G G_{i}$ if $f_{i} \leq f_{-i}$.

(ii) If $\pi^{d} / \pi^{m}<1 / 2$, then

- for $f_{i}, f_{-i} \leq \pi^{d}$, the equilibrium is $S E G$ if $f_{-i}<\pi^{m} / 2-\pi^{d}+f_{i}$, and $A G G_{i}$ if $f_{-i} \geq \pi^{m} / 2-\pi^{d}+f_{i}$.

- for $f_{i} \leq \pi^{d}$ and $f_{-i}>\pi^{d}$, the equilibrium is $A G G_{i}$.

- for $f_{i}, f_{-i} \in\left(\pi^{d}, \pi^{m} / 2\right]$, the equilibrium is $S E G$ if $f_{-i} \leq\left(\pi^{m}-\pi^{d}\right) / 2-f_{i}$ and $S T A_{i}$ if $f_{-i}>\left(\pi^{m}-\pi^{d}\right) / 2-f_{i}$.

- for $f_{i}>\pi^{d}$ and $f_{-i}>\pi^{m} / 2$, the equilibrium is $S T A_{i}$ if $f_{i} \leq f_{-i}$.

\section{Appendix B: Proof of Propositions}

Proof of Proposition 1. We first consider the case $\pi^{d} / \pi^{m} \geq 1 / 2$. From Appendix A, we know that an agglomeration equilibrium on platform $i$ exits only if $f_{i} \leq \pi^{d}$ and $f_{i} \leq f_{-i}$. The latter condition occurs because, in the second stage, sellers and buyers will coordinate on the equilibrium in which they all join the platform with the lower fee. This implies

\footnotetext{
${ }^{44}$ If both fees are above $\pi^{d}$ there is no restriction because the agglomeration equilibrium does not exist then.
} 
that, in the first stage, for all $f_{-i} \leq \pi^{d}$, platform $i$ gains from undercutting the competing platform. This induces agglomeration on platform $i$. The standard Bertrand logic then applies and platforms set $f_{A}^{\star}=f_{B}^{\star}=0$ in equilibrium.

Now we turn to the case $\pi^{d} / \pi^{m}<1 / 2$. It is evident from Appendix A that a segmentation equilibrium is played if fees are symmetric. As a consequence, in a candidate agglomeration equilibrium, the inactive platform must set a strictly positive fee. But this platform then has the incentive to lower its fee (but still keep it strictly positive) and induce segmentation. By doing so, it obtains a strictly positive profit, as half of the sellers join the platform. This argument holds for any $f_{A^{-}} f_{B^{-}}$combination, which induces agglomeration in the second stage. It follows that no agglomeration equilibrium exists for $\pi^{d} / \pi^{m}<1 / 2$.

Proof of Proposition 2. Consider the region of $\pi^{d} / \pi^{m}<1 / 2$. In a segmentation equilibrium, a seller active on platform $i$ obtains a profit of $\pi^{m} / 2-f_{i}$. Therefore, the highest possible fee that a platform can charge equals $\pi^{m} / 2$, leaving sellers with zero profits. We first determine the conditions under which an equilibrium with listing fees $\pi^{m} / 2$ exists. If both platforms charge $f_{i}=\pi^{m} / 2$, the only possible configuration in the second stage is the segmentation equilibrium. This follows because the profit that a seller obtains with agglomeration equals $\pi^{d}$, which is below the listing fee.

Suppose that platform $i$ deviates to induce an agglomeration equilibrium in the second stage. To do so, it has to charge $f_{i}^{d e v}=\pi^{d}-\epsilon$, where $\epsilon>0$ can be arbitrarily small. Since all buyers will agglomerate on platform $i$ if all sellers do, sellers earn then a small positive profit when agglomerating on platform $i$ but zero in the segmentation equilibrium. The deviation profit of platform $i$ is then (letting $\epsilon \rightarrow 0$ ) $\Pi_{i}^{d e v}=2 \pi^{d}$ since it obtains $\pi^{d}$ from each seller. Therefore, a deviation is not profitable if $\pi^{m} / 2 \geq 2 \pi^{d}$ or $\pi^{d} / \pi^{m} \leq 1 / 4$. It follows that in this region, a segmentation equilibrium with listing fees $\left(f_{A}^{\star}, f_{B}^{\star}\right)=$ $\left(\pi^{m} / 2, \pi^{m} / 2\right)$ is the unique equilibrium. Platforms' equilibrium profits are $\pi^{m} / 2$.

There cannot exist a segmentation equilibrium in which platforms charge fees less than $\pi^{m} / 2$. The reason is that, given the equilibrium played in stage 2 , a platform could then increase its fee slightly, still induce segmentation at stage 2, and obtain a higher profit.

Proof of Proposition 3. We first show the non-existence of a pure-strategy equilibrium. Consider the region of $1 / 4<\pi^{d} / \pi^{m}<1 / 2$. From Appendix A, we know that in this region a segmentation equilibrium will be played in the second stage if both platforms charge the same listing fees (conditional on these fees being lower than $\pi^{m} / 2$, which will always be fulfilled in equilibrium). From Proposition 2 it follows that platforms cannot extract the full profits from sellers because this would give each platform an incentive to deviate to a lower fee and induce agglomeration. We proceed by first determining the 
highest fee that platforms could charge to make such a downward deviation unprofitable. Suppose that both platforms charge a fee of $\pi^{m} / 2-x$. Each platform's profit is then $\pi^{m} / 2-x$, and a seller's profit is $x$. If platform $i$ deviates in order to attract all sellers and buyers in the second stage, it must set a fee such that $\pi^{d}-f_{i}^{d e v}>x$. The highest possible deviation fee is therefore $f_{i}^{d e v}=\pi^{d}-x-\epsilon$, leading to a deviation profit of (letting $\epsilon \rightarrow 0$ ) $2 \pi^{d}-2 x$. Such a deviation is unprofitable if $\pi^{m} / 2-x \geq 2 \pi^{d}-2 x$ or $x \geq 2 \pi^{d}-\pi^{m} / 2$. Hence, with an $x$ equal to $2 \pi^{d}-\pi^{m} / 2$, platforms prevent such a downward deviation. The resulting listing fee is then $f_{i}=\pi^{m} / 2-x=\pi^{m}-2 \pi^{d}$, and the platform's profit is also $\pi^{m}-2 \pi^{d}$.

To determine if listing fees $f_{i}=f_{j}=\pi^{m}-2 \pi^{d}$ can constitute an equilibrium, we need to check if a platform has an incentive to deviate by charging a higher listing fee (upward deviation). Suppose that platform $i$ charges $f_{i}=\pi^{m}-2 \pi^{d}$ and platform $j$ charges a deviation fee $f_{j}^{\text {dev }}>f_{i}$ such that segmentation is still the continuation equilibrium. To induce a segmentation equilibrium, we must have $\pi^{m} / 2-f_{j}^{d e v}>\pi^{d}-f_{i}=3 \pi^{d}-\pi^{m}$ (i.e., a seller's profit with segmentation must be higher than with agglomeration). Thus, the highest possible listing fee is $f_{j}^{d e v}=3 \pi^{m} / 2-3 \pi^{d}-\epsilon=3\left(\pi^{m} / 2-\pi^{d}\right)-\epsilon$, which yields a larger platform profit than $f_{i}=2\left(\pi^{m} / 2-\pi^{d}\right)$. As a consequence, a profitable upward deviation exists.

It follows that there is no equilibrium in pure strategies in the range of $1 / 4<\pi^{d} / \pi^{m}<$ $1 / 2$. The candidate equilibrium, which prevents downward deviations was $f_{i}=f_{j}=$ $\pi^{m}-2 \pi^{d}$; but then an upward deviation is profitable. In turn, for all listing fees above $\pi^{m}-2 \pi^{d}$, a downward deviation is profitable.

Randomization domain. From the analysis above, we know that in the range $1 / 4<$ $\pi^{d} / \pi^{m}<1 / 2$ for each $f_{j}$, platform $i$ has two best-response candidates: an upper bestresponse candidate, denoted by $f_{i}^{b{ }^{+}}$, which is higher than $f_{j}$ by a discrete amount and induces segmentation, and a lower best-response candidate, denoted by $f_{i}^{b r^{-}}$, which is lower than $f_{j}$ by a discrete amount and induces agglomeration. We will now show that there is a unique $f_{j}$ so that platform $i$ obtains the same profit with either best-response candidate. In addition, both candidates are increasing in $f_{j}$. Due to platform symmetry, this allows us to derive the randomization domain.

Suppose that platform $j$ sets a fee $f_{j}$. We now derive the best response of platform $i \neq j$. The upper best response $f_{i}^{b r^{+}}$induces segmentation with the highest possible fee. At this fee, sellers weakly prefer segmentation to agglomeration on $j$, which implies that the inequality $\pi^{m} / 2-f_{i}^{b r^{+}} \geq \pi^{d}-f_{j}$ is binding 45 This leads to a profit of $f_{i}^{b r^{+}}=$ $\pi^{m} / 2-\pi^{d}+f_{j}$. Instead, the optimal lower best response $f_{i}^{b r^{-}}$is the largest fee which induces agglomeration on platform $i$. Thus, we must have $\pi^{d}-f_{i}^{b r^{-}}>\pi^{m} / 2-f_{j}$. The

\footnotetext{
${ }^{45} \mathrm{We}$ assume that if sellers are indifferent between segmentation and agglomeration, they choose segmentation. As we will show below, this is consistent with equilibrium behavior.
} 
lowest upper bound of platform $i$ 's profit is then $2 f_{i}^{b r^{-}}=2\left(\pi^{d}-\pi^{m} / 2+f_{j}\right)$.

The two profits reported above are equal at $f_{j}=3 / 2 \pi^{m}-3 \pi^{d}$. Thus, there is a unique $f_{j}$ such that platform $i$ just prefers the upper to the lower best-response candidate. Platform $i$ 's best response to $f_{j} \leq 3 / 2 \pi^{m}-3 \pi^{d}$ is to induce segmentation at $f_{i}^{b r^{+}}=$ $\pi^{m} / 2-\pi^{d}+f_{j}=2 \pi^{m}-4 \pi^{d}$, which is increasing in $f_{j}$. Vice versa, for $f_{j}>3 / 2 \pi^{m}-3 \pi^{d}$, platform $i$ 's profit from the lower best-response candidate is larger than that from the upper best-response candidate because $2\left(\pi^{d}-\pi^{m} / 2+f_{j}\right)>\pi^{m} / 2-\pi^{d}+f_{j}$ for $\pi^{d}<\pi^{m} / 2$. This implies that its best response to $f_{j}>3 / 2 \pi^{m}-3 \pi^{d}$ is $f_{i}^{b r^{-}}=\pi^{d}-\pi^{m} / 2+f_{j}$, which is increasing in $f_{j}$. Hence, along platform $i$ 's best response, its maximal profit is reached at $f_{j}=3 / 2 \pi^{m}-3 \pi^{d}$ and is given by $f_{i}^{b r^{+}}=2 \pi^{m}-4 \pi^{d}$. By symmetry, this leads to an upper interval of the randomization domain equal to $f_{j} \in\left[3 / 2 \pi^{m}-3 \pi^{d}, 2 \pi^{m}-4 \pi^{d}\right]$. Analogously, the minimum of platform $i$ 's best response to $f_{j}$ is reached at $f_{j}=3 / 2 \pi^{m}-3 \pi^{d}$ and is given by $f_{i}^{b r^{-}}=\pi^{m}-2 \pi^{d}$. This leads to a lower interval of the randomization domain equal to $f_{j} \in\left[\pi^{m}-2 \pi^{d}, 3 / 2 \pi^{m}-3 \pi^{d}\right)$.

As a consequence, there is mixed-strategy equilibrium in which $f_{i}, f_{j} \in\left[\pi^{m}-2 \pi^{d}, 2 \pi^{m}-\right.$ $\left.4 \pi^{d}\right]$. The expected profit in this equilibrium is $3 \pi^{m} / 2-3 \pi^{d}$. This is because when charging this fee, a platform induces segmentation with a probability of (almost) 1.

In this mixed-strategy equilibrium, the highest listing fee is $2 \pi^{m}-4 \pi^{d}$. To ensure participation of sellers, the highest fee a platform can charge (in a segmentation equilibrium) is $\pi^{m} / 2$. Therefore, the equilibrium determined above is only valid if $2 \pi^{m}-4 \pi^{d} \leq \pi^{m} / 2$ or $\pi^{d} / \pi^{m} \geq 3 / 8$.

Mixing probabilities. In the range $3 / 8 \leq \pi^{d} / \pi^{m}<1 / 2$, platforms set fees $f_{i}, f_{j} \in$ $\left[\pi^{m}-2 \pi^{d}, 2 \pi^{m}-4 \pi^{d}\right]$, and the expected profit is $\Pi_{A}^{\star}=\Pi_{B}^{\star}=3 \pi^{m} / 2-3 \pi^{d}$.

Let $\delta \equiv \pi^{m} / 2-\pi^{d}$ and $\epsilon>0$ but infinitesimally small. Denote $\underline{f} \equiv 2 \delta, \tilde{f} \equiv 3 \delta$, and $\bar{f} \equiv 4 \delta$. Thus, the domain over which platforms mix is $[\underline{f}, \bar{f}]=[2 \delta, 4 \delta]$. For $i, j \in\{A, B\}$ and $i \neq j$, the platforms' best response functions are given by ${ }^{46}$

$$
\hat{f}_{i}\left(f_{j}\right)= \begin{cases}f_{j}+\delta, & \text { if } f_{j} \in[\underline{f}, \tilde{f}] \\ f_{j}-\delta-\epsilon, & \text { if } f_{j} \in(\tilde{f}, \bar{f}]\end{cases}
$$

We know that all fees in the mixing domain must give an expected profit of $3 \delta$, as otherwise platforms would not be indifferent between these fees. We need to distinguish between two intervals, a lower and an upper one. The lower interval consists of fees $f_{i} \in[2 \delta, 3 \delta)$ and the upper interval consist of fee $f_{i} \in[3 \delta, 4 \delta]$. The reason for this

\footnotetext{
${ }^{46}$ As sellers choose segmentation in the second stage when being indifferent between segmentation and agglomeration, the best response to $f_{j}$ marginally above $\tilde{f}$ is $f$, and the best response to $f_{j}=\tilde{f}$ is $\bar{f}$. Therefore, all boundaries of the mixing region are well defined. By contrast, if sellers chose agglomeration when indifferent, the upper bound would not be well defined, as $\bar{f}$ is never a best response (only $\bar{f}-\epsilon$ is). Therefore, sellers choosing segmentation as a continuation equilibrium when indifferent is consistent with equilibrium play of the full game.
} 
distinction is that in the lower interval, sellers may agglomerate on platform $i$ (i.e., this happens if $f_{j}>f_{i}+\delta$ ) but will never agglomerate on platform $j$. That is, if $f_{i}$ is in this lower interval, platform $i$ will always obtain a positive profit. By contrast, if $f_{i}$ is an element of the upper interval, with some probability sellers will choose to agglomerate on platform $j$-this occurs if platform $j$ charges $f_{j}<f_{i}-\delta$-and platform $i$ obtains no profit. Platform $i$ 's profit can then be written as

$$
\Pi_{i}\left(f_{i}, f_{j}\right)= \begin{cases}0, & \text { if } f_{i} \in\left(f_{j}+\delta, 4 \delta\right] \wedge f_{j} \in[2 \delta, 3 \delta) ; \\ f_{i}, & \text { if } f_{i} \in\left[\max \left\{2 \delta, f_{j}-\delta\right\}, \min \left\{f_{j}+\delta, 4 \delta\right\}\right] \wedge f_{j} \in[2 \delta, 4 \delta] \\ 2 f_{i}, & \text { if } f_{i} \in\left[2 \delta, f_{j}-\delta\right) \wedge f_{j} \in(3 \delta, 4 \delta] .\end{cases}
$$

Let us start with the case in which platform $i$ charges a fee in the lower interval - that is, $f_{i} \in[2 \delta, 3 \delta)$. Denote the cumulative density function with which platform $j$ mixes by $G_{1}\left(f_{j}\right)$. Platform $i$ 's profit with a fee in this lower interval is then given by (replacing $f_{i}$ by $f$ )

$$
G_{1}(f+\delta) f+\left(1-G_{1}(f+\delta)\right) 2 f
$$

In equilibrium, this expression must be equal to $3 \delta$, yielding

$$
G_{1}(f+\delta) f+\left(1-G_{1}(f+\delta)\right) 2 f=3 \delta .
$$

This equation determines the mixing probabilities of platform $j$ in its upper interval. This is because only if platform $j$ sets a fee above $f+\delta$ (which happens with probability $1-G_{1}(f+\delta)$ ), sellers will agglomerate on platform $i$. Such a fee must necessarily be in the upper interval.

If platform $i$ charges a fee in the upper interval — that is, $f_{i} \in[3 \delta, 4 \delta]$-its profit is

$$
G_{1}(f-\delta) 0+\left(1-G_{1}(f-\delta)\right) f=3 \delta
$$

This equation determines the mixing probability in the lower interval.

Let us first look at (4). We can substitute $h \equiv f+\delta$ to get

$$
G_{1}(h)(h-\delta)+\left(1-G_{1}(h)\right) 2(h-\delta)=3 \delta .
$$

Recall that (4) was relevant for $f$ in the lower range and, since $h=f+\delta$, these are exactly the fees in the upper interval. Solving $(6)$ for $G_{1}(h)$ gives

$$
G_{1}(h)=\frac{2 h-5 \delta}{h-\delta}
$$

It is easy to check that $G_{1}(4 \delta)=1$. 
Now we turn to (5). Here, we can substitute $h \equiv f-\delta$ representing that $h$ is now in the lower interval. We obtain

$$
\left(1-G_{1}(h)\right)(h+\delta)=3 \delta
$$

Solving (8) for $G_{1}(h)$ gives

$$
G_{1}(h)=\frac{h-2 \delta}{h+\delta}
$$

It is easy to check that $G_{1}(2 \delta)=0$. Using (7) and (9), we obtain $\lim _{h \searrow 3 \delta}=1 / 2$ and $\lim _{h \nearrow 3 \delta}=1 / 4$. This implies the existence of a mass point with mass $1 / 4$ at a fee equal to $3 \delta 47$

The resulting mixing probability is characterized by the cumulative distribution function

$$
G_{1}(f)= \begin{cases}\frac{f-2 \delta}{f+\delta}, & \text { if } f \in[2 \delta, 3 \delta) \\ \frac{2 f-5 \delta}{f-\delta}, & \text { if } f \in[3 \delta, 4 \delta]\end{cases}
$$

Because the distribution is not absolutely continuous with respect to the Lebesgue measure, it fails to have a density. Nevertheless, we define a generalized density, which is a generalized function (since it will be comprised of a dirac delta function) such that integration against this generalized function yields the correct desired probabilities. The corresponding probability density function is given by

$$
g_{1}(f)=G_{1}^{\prime}(f)+\frac{1}{4} \delta^{D}(f-3 \delta)
$$

where

$$
G_{1}^{\prime}(f)= \begin{cases}\frac{3 \delta}{(f+\delta)^{2}}, & \text { if } f \in[2 \delta, 3 \delta) \\ \frac{3 \delta}{(f-\delta)^{2}}, & \text { if } f \in[3 \delta, 4 \delta]\end{cases}
$$

and $\delta^{D}\left(f-f_{0}\right)$ denotes Dirac's delta function which is 0 everywhere except for $f_{0}$ where it is $\infty$. Furthermore, $\int \delta^{D}\left(f-f_{0}\right) d f=1$. Inserting $\delta=\pi^{m} / 2-\pi^{d}$ yields the result stated in the proposition.

Proof of Proposition 4. As shown in the proof of the previous proposition, a pure-strategy equilibrium in the region $1 / 4<\pi^{d} / \pi^{m}<1 / 2$ does not exist. Furthermore, for $3 / 8 \leq$ $\pi^{d} / \pi^{m}<1 / 2$, there exists a mixed-strategy equilibrium which has an upper bound of the randomization domain equal to $2 \pi^{m}-4 \pi^{d}$. This equilibrium cannot exist in the range

\footnotetext{
${ }^{47}$ Intuitively, equation 5 requires a sufficiently high probability of $f-\delta$ being close to $3 \delta$ since, as otherwise setting $f$ close to $4 \delta$ would lead to zero profit "too often".
} 
$1 / 4<\pi^{d} / \pi^{m}<3 / 8$ because $2 \pi^{m}-4 \pi^{d}$ would then be larger than $\pi^{m} / 2$. We next derive the randomization domain of the mixed-strategy equilibrium with an upper bound of $\pi^{m} / 2$.

Randomization domain. Suppose that platform $j$ sets $f_{j}=\pi^{m} / 2$. The best response of platform $i$ is then to set $f_{i}$ to induce agglomeration in the second stage. To do so, it needs to set $f_{i}=\pi^{d}-\epsilon$. The best response of platform $j$ is to marginally reduce its fee to $\pi^{m} / 2-\epsilon$ and induce a segmentation again, and so on. This goes on until platform $i$ sets the lowest fee in the randomization domain, denoted by $\underline{f}^{l}$. This is the fee at which platform $i$ is better off by raising its fee to the highest fee $\pi^{m} / 2$ and induce segmentation instead of marginally reducing it to induce agglomeration. Its segmentation profit is then $\pi^{m} / 2$. Hence, the lowest fee $\underline{f}^{l}$ is given by $2 \underline{f}^{l}=\pi^{m} / 2$ or, equivalently, $\underline{f}^{l}=\pi^{m} / 4$. This fee makes sellers exactly indifferent between agglomeration on platform $i$ and segmentation if platform $j$ charges a fee such that $\pi^{d}-\underline{f}^{l}=\pi^{m} / 2-f_{j}$ or, equivalently, $f_{j}=3 \pi^{m} / 4-\pi^{d}$.

Finally, note that a fee $\pi^{d}-\epsilon$ (i.e., the fee that induces agglomeration if the rival platform charges the highest fee) is strictly lower than $3 \pi^{m} / 4-\pi^{d}$ (i.e., the fee at which the rival stops lowering its fee and instead raises the fee to the highest one) since we are in the range $\pi^{d} / \pi^{m}<3 / 8$. Therefore, the upper bound of the lower interval is below the lower bound of the upper interval. It follows that there are two disjoint sets of mixing intervals. The upper one $\left[3 \pi^{m} / 4-\pi^{d}, \pi^{m} / 2\right]$, in which each fee is a best response to a fee in the lower interval $\left[\pi^{m} / 4, \pi^{d}\right)$. In turn, each fee in the lower interval is a best response to a fee in the upper interval.

To summarize the above analysis, in the range $1 / 4<\pi^{d} / \pi^{m}<3 / 8$, there is a mixedstrategy equilibrium with fees $f_{i} \in\left[\pi^{m} / 4, \pi^{d}\right) \cup\left[3 \pi^{m} / 4-\pi^{d}, \pi^{m} / 2\right]$. For any chosen fee, the expected profit in this range must be $3 \pi^{m} / 4-\pi^{d}$. As above, this is because setting a fee equal to $3 \pi^{m} / 4-\pi^{d}$ induces segmentation with a probability of (almost) 1 .

Mixing Probabilities. Let $\eta \equiv \pi^{d}-\pi^{m} / 4, \delta \equiv \pi^{m} / 2-\pi^{d}$ and $\epsilon>0$ but infinitesimally small. Denote $\underline{f}^{l} \equiv \pi^{m} / 4, \bar{f}^{l} \equiv \pi^{d}, \underline{f}^{u} \equiv 3 \pi^{m} / 4-\pi^{d}$, and $\bar{f}^{p} \equiv \pi^{m} / 2$ such that the domain of interest can be expressed as $f_{i} \in\left[\underline{f}^{l}, \bar{f}^{l}\right) \cup\left[\underline{f}^{u}, \bar{f}^{u}\right]$. Using $\eta$ and $\delta$, the mixing domain can be written as $f_{i} \in[\eta+\delta, 2 \eta+\delta) \cup[\eta+2 \delta, 2 \eta+2 \delta]$. For $i, j \in\{A, B\}$ and $i \neq j$, the corresponding best response function is given by

$$
\hat{\hat{f}}_{i}\left(f_{j}\right)= \begin{cases}f_{j}+\delta, & \text { if } f_{j} \in\left[\underline{f}^{l}, \bar{f}^{l}\right) \\ f_{j}+\eta, & \text { if } f_{j}=\underline{f}^{u} \\ f_{j}-\delta-\epsilon, & \text { if } f_{j} \in\left(\underline{f}^{u}, \bar{f}^{u}\right] .\end{cases}
$$

We know that all fees in the mixing domain must give an expected profit of $\underline{f}^{u}=$ $(3 / 4) \pi^{m}-\pi^{d}=\eta+2 \delta$.

We now proceed analogously to the proof of Proposition 3. If platform $i$ charges a 
fee in the lower interval - that is, $f_{i} \in\left[\underline{f}^{l}, \bar{f}^{l}\right.$ ) -we obtain an equation analogous to (4), given by

$$
G_{2}(f+\delta) f+\left(1-G_{2}(f+\delta)\right) 2 f=\eta+2 \delta .
$$

This equation determines the mixing probabilities in the upper range.

In case platform $i$ charges a fee in the upper range, that is, $f_{i} \in\left[\underline{f}^{u}, \bar{f}^{u}\right]$, the equation is

$$
G_{2}(f-\delta) 0+\left(1-G_{2}(f-\delta)\right) f=\eta+2 \delta .
$$

This equation determines the mixing probability in the lower range.

Let us first look at $(10)$. Substituting $h \equiv f+\delta$ and solving for $G_{2}(h)$ gives

$$
G_{2}(h)=\frac{2 h-4 \delta-d}{h-\delta} .
$$

It is easy to check that $\lim _{f \searrow \underline{f}^{u}} G_{2}(f)=\lim _{f \searrow \eta+2 \delta} G_{2}(f)=\delta /(\eta+\delta)$. Moreover, $\lim _{f \nearrow \bar{f}^{u}} G_{2}(f)=$ $\lim _{f / 2 \delta+2 \eta} G_{2}(f)=3 \delta /(2 \delta+\eta)<1$. The latter implies the existence of a mass point with mass $1-3 \delta /(2 \delta+\eta)=(\delta-\eta) /(2 \eta+\delta)$ at a fee equal to $\bar{f}^{u}=2(\eta+\delta) 4$

Consider (11). We substitute $h \equiv f-\delta$. Thus, $h$ is now in the lower range. Solving for $G_{2}(h)$ gives

$$
G_{2}(h)=\frac{h-\eta-\delta}{h+\delta} .
$$

It is easy to check that $G_{2}(\underline{f})=G_{2}(\eta+\delta)=0$, whereas $\lim _{f \nearrow \bar{f}^{l}} G_{2}(f)=\lim _{f \nearrow 2 \eta+\delta} G_{2}(f)=$ $\delta /(2(\eta+\delta))$. Note that $\lim _{f \bar{f}^{l}} G_{2}(f)=\delta /(2(\eta+\delta))<\delta /(\eta+\delta)=\lim _{f \backslash \underline{f}^{u}} G_{2}(f)$, which implies the existence of a second mass point with mass $\delta /(2(\eta+\delta))$ at a fee equal to $\underline{f}^{u}=\eta+2 \delta$.

The resulting mixing probability is characterized by a cumulative distribution function of

$$
G_{2}(f)= \begin{cases}\frac{f-\eta-\delta}{f+\delta}, & \text { if } f \in[\eta+\delta, 2 \eta+\delta) ; \\ \frac{2 f-\eta-4 \delta}{f-\delta}, & \text { if } f \in[\eta+2 \delta, 2(\eta+\delta)) \\ 1, & \text { if } f=2(\eta+\delta) .\end{cases}
$$

The corresponding generalized density is given by

$$
g_{2}(f)=G_{2}^{\prime}(f)+\frac{\eta}{2(\eta+\delta)} \delta^{D}(f-(\eta+2 \delta))+\frac{\delta-\eta}{(2 \eta+\delta)} \delta^{D}(f-(2(\eta+\delta))),
$$

\footnotetext{
${ }^{48}$ Intuitively, in order to satisfy $(10)$, there must be a positive probability of inducing an agglomeration equilibrium and receiving $2 f$ in the lower range even for $f=\bar{f}^{l}$. This is achieved by a mass point at $h=\bar{f}^{u}$.
} 
where

$$
G_{2}^{\prime}(f)= \begin{cases}\frac{\eta+2 \delta}{(f+\delta)^{2}}, & \text { if } f \in[\eta+\delta, 2 \eta+\delta) ; \\ \frac{\eta+2 \delta}{(f-\delta)^{2}}, & \text { if } f \in[\eta+2 \delta, 2(\eta+\delta)),\end{cases}
$$

and $\delta^{D}\left(f-f_{0}\right)$ denotes Dirac's delta function. Replacing $\eta$ and $\delta$ by their respective definitions yields the result stated in the proposition.

Proof of Proposition 5. In stage 4, both multi-homing and single-homing buyers make their optimal buying decisions, given the prices charged by sellers in the third stage. In the third stage, the pricing equilibrium in the product market may be different than with single-homing buyers. If both sellers in a category are on the same platform, they will still charge a price of $p^{d}$ in equilibrium and obtain a profit of $\pi^{d}$ per buyer. Similarly, if only one seller is active, this seller sets its price equal to $\pi^{m}$ and earns $\pi^{m}$ per buyer. However, if sellers segment and one is active on platform $A$ and the other one on platform $B$, sellers no longer charge $p^{m}$. The reason is that a fraction $\alpha$ of buyers (i.e., the multi-homers) is informed about both offers. Therefore, the price charged by a seller depends on how many buyers are informed about both offers. We denote the price charged by a seller in this situation by $p(\alpha)$, with $p(\alpha) \in\left[\min \left\{p^{d}, p^{m}\right\}, \max \left\{p^{d}, p^{m}\right\}\right]$, and the respective per-buyer profit by $\pi(\alpha)$, with $\pi^{d} / \pi^{m} \leq \pi(\alpha) / \pi^{m} \leq 1$.

Turning to the second stage, we know that profits in an agglomeration and a standalone equilibrium are unchanged. This is not true for the segmentation equilibrium. If sellers segment, the total number of buyers for each seller is $(1+\alpha) / 2$, as explained in the main text. The profit of a seller active on platform $i$ is then $\pi(\alpha)(1+\alpha) / 2-f_{i}$. If the seller deviates and becomes active on platform $-i$, it obtains a profit of $\pi^{d}(1+\alpha) / 2-f_{-i}$. It follows that there is no deviation incentive if

$$
f_{i} \leq \min \left\{\left(\pi(\alpha)-\pi^{d}\right) \frac{(1+\alpha)}{2}+f_{-i}, \pi(\alpha) \frac{(1+\alpha)}{2}\right\}
$$

In contrast to the case with single-homing buyers where the relevant condition was given by (3), the buyer mass $1 / 2$ is now replaced by $(1+\alpha) / 2$ and the monopoly profit $\pi^{m}$ is replaced by $\pi(\alpha)$. Proceeding in the same way as in Appendix A, we obtain that in the second stage there is unique equilibrium and the conditions for the agglomeration, the segmentation, and stand-alone equilibrium to occur are still the same as given there, with $\pi^{m} / 2$ replaced by $\pi(\alpha)(1+\alpha) / 2$.

We can now move to the first-stage. Following the same argument as in the proof of Proposition 1, we obtain that in the range $\pi^{d} \geq \pi(\alpha)(1+\alpha) / 2$ an agglomeration equilibrium with fees $f_{i}=f_{-i}=0$ is the unique equilibrium. Similarly, if both platforms charge a fee of $\pi(\alpha)(1+\alpha) / 2$, the only equilibrium is that sellers segment, and a platform's profit 
equals $\pi(\alpha)(1+\alpha) / 4$. A platform has no incentive to deviate from this fee combination, if

$$
\pi^{d} \leq \pi(\alpha) \frac{1+\alpha}{4}
$$

Hence, in this range, the unique equilibrium involves $f_{i}=f_{-i}=\pi(\alpha)(1+\alpha) / 2$ and a segmentation equilibrium occurs.

It is evident that the regions are the same as in case where $\alpha=0$ with the difference that $\pi^{m} / 2$ is replaced by $\pi(\alpha)(1+\alpha) / 2$. The same logic applies for the region

$$
\pi(\alpha) \frac{1+\alpha}{2}>\pi^{d}>\pi(\alpha) \frac{1+\alpha}{4} .
$$

By following the same steps as in the proofs of Propositions 1 through 4 , we obtain the same results as in those propositions.

Proof of Proposition 6. Stage 4 works in the same way as without multi-homing: given sellers' prices, buyers make their optimal purchasing decisions. In stage 3, due to the possibility of multi-homing, new competition situations can occur. As mentioned in the main text, these are that, in a category, either both sellers multi-home or that only one seller multi-homes whereas the other single-homes on platform $i$. In the former situation, regardless of the distribution of buyers, all buyers are informed about both sellers' offers. It follows that sellers in the third stage will set a price of $p^{d}$, leading to a profit of $\pi^{d}-f_{A}-f_{B}$ for both sellers. In the latter situation, sellers compete in an asymmetric way, as the multi-homing seller reaches all buyers, whereas the singlehoming seller reaches only buyers on platform $i$. If platform $i$ is host to $x \in(0,1)$ buyers, we denote the prices set by the sellers in the third-stage equilibrium by $p^{S H}(x)$ for the single-homing seller and by $p^{M H}(x)$ for the multi-homing seller. The respective per-buyer profits are $\pi^{S H}(x)$ and $\pi^{M H}(x)$, respectively, which implies that the profits of the two sellers are $x \pi^{S H}(x)-f_{i}$ and $\pi^{M H}(x)-f_{A}-f_{B} 49$

We turn to the second stage. We first determine the conditions under which the different equilibrium configurations determined in the game with single-homing are still Nash equilibria with multi-homing. First, as before, agglomeration on platform $i$ is an equilibrium if $f_{i} \leq \pi^{d}$. As there is no buyer on the other platform, the possibility to multi-home does not change the outcome. The same holds true for the stand-alone equilibrium on platform $i$, which is a Nash equilibrium whenever $\pi^{d}<f_{i} \leq \pi^{m}$. Turning to the segmentation equilibrium, in addition to the deviations considered in Appendix A, a seller can now also choose to multi-home. This is not profitable if and only if

\footnotetext{
${ }^{49}$ The per-buyer profit of the multi-homing seller, $\pi^{M H}(x)$, is a weighted average of the profit obtained from buyers on platform $i$ who are informed about both offers and the profit from buyers on platform $-i$ who only observe the multi-homing seller's offer.
} 
$\pi^{m} / 2-f_{i} \geq \pi^{M H}-f_{i}-f_{-i}$, where $\pi^{M H} \equiv \pi^{M H}(1 / 2)$. Therefore, the conditions under which a segmentation equilibrium exists are more demanding than in the case of singlehoming; they are given by

$$
f_{i} \leq \min \left\{\frac{\pi^{m}-\pi^{d}}{2}+f_{-i}, \frac{\pi^{m}}{2}\right\} \quad \text { and } \quad f_{-i} \geq \pi^{M H}-\frac{\pi^{m}}{2}
$$

In addition to these equilibria which involve single-homing of sellers, there can also be equilbria which involve multi-homing along the equilibrium path. One is a full multihoming equilibrium in which both sellers multi-home and buyers split evenly on platforms. This is a Nash equilibrium if no seller has an incentive to deviate to single-homing - that is, $\pi^{d}-f_{i}-f_{-i} \geq \pi^{S H} / 2-f_{i}$ and, thus, in equilibrium, $f_{-i} \leq \pi^{d}-\pi^{S H} / 2$.

In addition, there can be a partial multi-homing equilibrium with the following structure: In each category, one seller multi-homes and the other one single-homes. A singlehoming seller is active on platform $A$ in half of the categories and on platform $B$ in the other half of the categories. Buyers are indifferent, as each platform has, in expectation, the same number of sellers in the buyers' preferred category and, therefore, buyers will split evenly. A multi-homing seller's profit is $\pi^{M H}-f_{A}-f_{B}$ and the profit of a seller single-homing on platform $i$ is $\pi^{S H} / 2-f_{i}$.

We determine the conditions under which this configuration is a Nash equilibrium. First, any single-homing seller must earn non-negative profits - that is, $\pi^{S H} / 2-f_{i} \geq 0$. Second, it must be optimal for any such sellers to single-home on platform $i$ instead of single-homing on platform $-i$ - that is, $\pi^{S H} / 2-f_{i} \geq \pi^{S H} / 2-f_{-i}$. Third, single-homing must be better than multi-homing for this seller - that is, $\pi^{S H} / 2-f_{i} \geq \pi^{M H}-f_{i}-f_{-i}$. Moreover, the multi-homing seller must be better off with multi-homing than with singlehoming on platform $i$ or $-i$. These conditions are satisfied if $\pi^{M H}-f_{i}-f_{-i} \geq \pi^{d} / 2-f_{i}$ and $\pi^{M H}-f_{i}-f_{-i} \geq \pi^{m} / 2-f_{-i}$.

The same conditions must also hold with $f_{i}$ and $f_{-i}$ interchanged because, in the partial multi-homing equilibrium, one half of the single-homing sellers are on platform $A$ and the other half on platform $B$. Importantly, this implies that $\pi^{S H} / 2-f_{-i} \geq \pi^{S H} / 2-f_{i}$. Taken together with the condition $\pi^{S H} / 2-f_{i} \geq \pi^{S H} / 2-f_{-i}$ (which has been derived above), this shows that a partial multi-homing equilibrium can only exist if $f_{i}=f_{-i}{ }^{50}$ Using $f_{i}=f_{-i}$ together with all other conditions derived above, we obtain partial multihoming is a Nash equilibrium if and only if

$$
f_{i}=f_{-i}, \quad f_{i} \leq \frac{\pi^{S H}}{2}, \quad \text { and } \quad \pi^{d}-\frac{\pi^{S H}}{2} \leq f_{i} \leq \pi^{M H}-\frac{\pi^{m}}{2}
$$

\footnotetext{
${ }^{50}$ Therefore, in the $f_{A^{-}} f_{B^{-}}$diagram presented in Figure 1, the partial multi-homing equilibrium can only exist on the 45-degree line.
} 
As follows from (14) and (15), the partial multi-homing equilibrium and the segmentation equilibrium co-exist if and only if $f_{i}=f_{-i}=\pi^{M H}-\pi^{m} / 2$.

Next, we apply our refinement. First, it is easy to see that the full multi-homing equilibrium is never coalition-proof. Take the coalition of all sellers and all buyers on platform $i$, and consider a deviation in which all buyers go to platform $-i$ and sellers single-home on platform $-i$. Then buyers get the same utility as with full multi-homing but sellers are better off as they receive a profit of $\pi^{d}-f_{-i}>\pi^{d}-f_{i}-f_{-i}$. Therefore, full multi-homing never survives our refinement.

Turning to the partial multi-homing equilibrium, we determine the conditions for coalition-proofness of this equilibrium. First, single-homing sellers on platform $i$ can form a coalition with buyers on platform $i$ and deviate to be active only on platform $-i$. Buyers are then better off, as they are informed about all offers on platform $-i$ and sellers compete in all categories whereas sellers are only better off if $\pi^{d}-f_{-i}>\pi^{S H} / 2-f_{i}$. As the partial multi-homing Nash equilibrium only exists for $f_{i}=f_{-i}$, we obtain that such a deviation is not profitable if $\pi^{S H} / 2 \geq \pi^{d}$. Second, multi-homing sellers can form a coalition with all buyers on the platform where the sellers are monopolists (platform $i$, say) and single-home on platform $-i$. Buyers are better off, as sellers compete on platform $-i$, whereas the originally multi-homing sellers are better off if and only if $\pi^{d}-f_{-i}>\pi^{M H}-f_{i}-f_{-i}$. Therefore, this deviation is not profitable if $f_{i} \leq \pi^{M H}-\pi^{d}$. (The other deviations by sellers do not involve coalitions and, therefore, are already captured by the conditions for the Nash equilibrium to exist.) We now combine these conditions with the ones derived in 15 . Since coalition-proofness requires $\pi^{S H} / 2 \geq \pi^{d}$, the lower bound on $f_{i}$ derived in (15) would be weakly negative and, thus, can be replaced by zero. In addition, the condition $\pi^{S H} / 2 \geq \pi^{d}$ also implies $\pi^{d} / \pi^{m} \leq 1 / 2$ and, therefore, $\pi^{M H}-\pi^{m} / 2 \leq \pi^{M H}-\pi^{d}$. Hence, the upper bound on $f_{i}$ is $\min \left\{\pi^{S H} / 2, \pi^{M H}-\pi^{m} / 2\right\}$. To sum up, the partial multi-homing equilibrium is coalition-proof only if

$$
f_{i}=f_{-i}, \quad \frac{\pi^{S H}}{2} \geq \pi^{d}, \quad \text { and } \quad f_{i} \leq \min \left\{\frac{\pi^{S H}}{2}, \pi^{M H}-\frac{\pi^{m}}{2}\right\}
$$

Next, we determine whether the partial multi-homing equilibrium is in fact selected in the second stage, given that other equilibrium configurations are coalition-proof as well. From above, we know that it exists together with the segmentation equilibrium if and only if $f_{i}=f_{-i}=\pi^{M H}-\pi^{m} / 2$. The segmentation equilibrium is then also coalition-proof and preferred by sellers over the agglomeration equilibrium if $\pi^{m} / 2>\pi^{d}$ (see Appendix A). The profit of a seller in the segmentation equilibrium is $\pi^{m} / 2-f_{i}=$ $\pi^{m}-\pi^{M H}$. Instead, in the partial multi-homing equilibrium, a single-homing seller's profit is $\pi^{S H} / 2-f_{i}=\left(\pi^{S H}+\pi^{m}\right) / 2-\pi^{M H}$, which is strictly below the one in the segmentation equilibrium. A multi-homing seller's profit is $\pi^{M H}-2 f_{i}=\pi^{m}-\pi^{M H}$ and, therefore, the 
same as in the segmentation equilibrium. It follows that the segmentation equilibrium profit-dominates the partial multi-homing equilibrium. Hence, if $\pi^{m} / 2>\pi^{d}$, the partialmulti-homing equilibrium is selected in stage 2 if (16) holds, with the strengthening of the last condition to $f_{i} \leq \pi^{S H} / 2$ and $f_{i}<\pi^{M H}-\pi^{m} / 2$.

If instead $\pi^{m} / 2 \leq \pi^{d}$, the partial-multi-homing equilibrium may co-exist with the agglomeration equilibrium. Because the partial multi-homing equilibrium exists only if $\pi^{S H} / 2 \geq \pi^{d}$ and both fees are the same, the single-homing seller is better off in the partial multi-homing equilibrium. Since $\pi^{M H} \geq \pi^{S H}$, the multi-homing seller is better off as well. Hence, if $\pi^{m} / 2 \leq \pi^{d}$, the partial multi-homing equilibrium will be chosen in the second stage whenever 16 is fulfilled.

If the partial multi-homing equilibrium does not exist, for $f_{i}, f_{-i} \geq \pi^{M H}-\pi^{m} / 2$, the same analysis to select an equilibrium as in Appendix A applies, as in this case the same equilibria exist as without multi-homing. If instead one or both fees are lower than $\pi^{M H}-\pi^{m} / 2$, it follows from (14) that a segmentation equilibrium does not exist. However, we know from the analysis in Appendix A (see also Figure 1) that in this region either an agglomeration or a stand-alone equilibrium prevails, depending on parameters. It follows that, off the diagonal, there is a unique equilibrium in the second stage even with seller multi-homing, given our selection criterion.

We turn to the first stage. Let us first consider the case $\pi^{d} / \pi^{m} \geq 1 / 2$. This implies that $\pi^{d}>\pi^{S H} / 2$, as $\pi^{m}>\pi^{S H}$. Therefore, the partial multi-homing equilibrium does not exist in this case. It follows that the analysis of the proof of Proposition 1 applies, leading to $f_{A}=f_{B}=0$ in equilibrium, and buyers and sellers play an agglomeration equilibrium in the second stage.

Second, consider the case $\pi^{d} / \pi^{m} \leq 1 / 4$. In the pure-strategy segmentation equilibrium of Proposition 2, platforms set $f_{A}=f_{B}=\pi^{m} / 2$. As $\pi^{m} / 2>\pi^{M H}-\pi^{m} / 2$, due to the fact that $\pi^{m}>\pi^{M H}$, the segmentation equilibrium exists in this case. From the analysis of the second stage, it follows that the partial multi-homing equilibrium does not exist then, and from the proof of Proposition 2 it follows that the pure-strategy segmentation equilibrium is the unique equilibrium in this case. This establishes the first part of the proposition.

Turning to the range $1 / 2>\pi^{d} / \pi^{m}>1 / 4$, we first consider the situation in which $\pi^{d}>\pi^{S H} / 2$, that is, the partial multi-homing equilibrium does not exist. We know from above that for $\pi^{M H} \leq \pi^{m} / 2$, the segmentation equilibrium exists, which implies that the equilibrium is the same mixed-strategy equilibrium as the one characterized in Propositions 3 and 4. By contrast, for $\pi^{M H}>\pi^{m} / 2$, a segmentation equilibrium does not exist. We will now check under which conditions the possibility to multi-home breaks the mixed-strategy equilibrium of Propositions 3 and 4 . This equilibrium exists if the circle of best responses described in the proofs of these propositions works in the same 
way if sellers can multi-home. However, this circle does no longer exist if one of the fees in the mixing range is below $\pi^{M H}-\pi^{m} / 2$. The reason is as follows: Suppose platform $i$ sets a fee below $\pi^{M H}-\pi^{m} / 2$. Platform $-i$ 's best response in case of single-homing sellers was to set a higher fee to induce segmentation. However, inducing segmentation is no longer possible with multi-homing sellers. As a consequence, the best response of platform $-i$ to a listing fee of $f_{i}$ below $\pi^{M H}-\pi^{m} / 2$ is to undercut this fee slightly to induce an agglomeration equilibrium on platform $-i$ in the second stage. The lowering of fees then leads to the agglomeration equilibrium with $f_{A}=f_{B}=0$.

It remains to be checked under which conditions the lowest fee in the mixing range is below $\pi^{M H}-\pi^{m} / 2$. Starting with the first mixing region we obtain that this holds if $\pi^{M H}-\pi^{m} / 2>\pi^{m}-2 \pi^{d}$ or, equivalently,

$$
\pi^{M H}>\frac{3 \pi^{m}}{2}-2 \pi^{d}
$$

If this inequality holds, then $\pi^{M H}$ is also larger than $\pi^{m} / 2$, implying that any equilibrium features $f_{A}=f_{B}=0$ and agglomeration prevails at the second stage. Instead, if $\pi^{M H} \leq$ $3 \pi^{m} / 2-2 \pi^{d}$, the unique equilibrium is the mixed-strategy one, as reported in Proposition 3.

Proceeding in the same way for the second mixing region, we obtain that for

$$
\pi^{M H}>\frac{3 \pi^{m}}{4}
$$

any equilibrium features $f_{A}=f_{B}=0$ and agglomeration, whereas for $\pi^{M H} \leq 3 \pi^{m} / 4$, the unique equilibrium is the mixed-strategy one, as reported in Proposition 4.

Second, we consider the situation $\pi^{d} \leq \pi^{S H} / 2$. The partial multi-homing equilibrium then exists for fees $f_{A}=f_{B}$, with $f_{i} \leq \pi^{S H} / 2$ and $f_{i}<\pi^{M H}-\pi^{m} / 2, i=A, B$. The profit of each platform is $3 / 2 f_{i}$. However, each platform then has an incentive to lower its fee slightly. This induces agglomeration (as the segmentation equilibrium does not exist for $f_{i}<\pi^{M H}-\pi^{m} / 2$ ). The resulting profit of the platform with the lower fee (platform $i$, say) is then $2 f_{i}$. Hence, undercutting is profitable. As a consequence, if a partial multi-homing equilibrium exists in the full game, it can only occur with $f_{A}=f_{B}=0$. But, then, the same mechanism as described for the case $\pi^{d}>\pi^{S H} / 2$ occurs. The mixedstrategy equilibrium is the unique equilibrium in the first stage for the same region as in case $\pi^{d}>\pi^{S H} / 2$. In the other region, in equilibrium, platforms set their fees equal to zero. However, in contrast to the case above, for $\pi^{d} \leq \pi^{S H} / 2$, buyers and sellers in the second stage play the partial multi-homing equilibrium.

Proof of Proposition 7 . The third and fourth stage play out similarly as in the case with listing fees. In the fourth stage, buyers buy according to their demand functions, that 
is, a buyer active on platform $i$ either faces a seller price of $p^{m}\left(\phi_{i}\right)$ or $p^{d}\left(\phi_{i}\right)$, depending on the number on sellers on platform $i$, and then buys the respective number of goods $D^{m}\left(\phi_{i}\right)$ or $D^{d}\left(\phi_{i}\right)$. In stage 3 , a seller on platform $i$ sets $p^{m}\left(\phi_{i}\right)$ or $p^{d}\left(\phi_{i}\right)$, depending on the number of rival sellers (either 0 or 1 ) active on the platform in the seller's product category.

We turn to the second stage. Following the same arguments as in case of listing fees, there potentially exist three types of equilibria with per-transaction fees: agglomeration equilibria, segmentation equilibria, and stand-alone equilibria. In an agglomeration equilibrium on platform $i$, a seller's profit is $\pi^{d}\left(\phi_{i}\right)$, whereas in a segmentation equilibrium, the seller's profit is $\pi^{m}\left(\phi_{i}\right) / 2$. In a stand-alone equilibrium on platform $i$, the profit of an active seller is $\pi^{m}\left(\phi_{i}\right)$ and the one of an inactive seller is 0. However, since platforms charge per-transaction fees, if $\phi_{i}$ is such that $D^{m}\left(\phi_{i}\right)>0$ and thereby also $\pi^{m}\left(\phi_{i}\right)>0$, also the inactive seller in each category could make a positive profit by becoming active on platform $i$. The reason is that for $D^{m}\left(\phi_{i}\right)$ to be positive, $\phi_{i}$ must be below the intercept of the demand curve. This implies that also in duopoly sellers will charge prices such that $D^{d}\left(\phi_{i}\right)>0$, leading to $\pi^{d}\left(\phi_{i}\right)>0$. Since platforms in the first stage will never optimally charge a fee which leads to zero demand for sellers, as this implies zero profits also for platforms, we can restrict attention to those subgames in the second stage in which fees satisfy $D^{d}\left(\phi_{i}\right)>0.51$ For such fees, a stand-alone equilibrium does not exist in the second stage and, thus, will never occur along the equilibrium path of the full game.

Next, we determine the equilibrium that is played in the second stage, given our selection criterion. First, consider the case $\pi^{d}(0) / \pi^{m}(0) \geq 1 / 2$. Due to the assumption $\partial \pi^{m}\left(\phi_{i}\right) / \partial \phi_{i} \leq \partial \pi^{d}\left(\phi_{i}\right) / \partial \phi_{i} \leq 0$, the condition $\pi^{d}(0) / \pi^{m}(0) \geq 1 / 2$ implies that $\pi^{d}\left(\phi_{i}\right) / \pi^{m}\left(\phi_{i}\right) \geq 1 / 2 \forall \phi_{i}$. In this case, a segmentation equilibrium cannot exist in the second stage. The reason is that a coalition of all sellers and buyers on the platform with the higher fee have the incentive to deviate to the rival platform. It follows that for $\pi^{d}(0) / \pi^{m}(0) \geq 1 / 2$ only an agglomeration equilibrium exists.

Second, suppose that $\pi^{d}(0) / \pi^{m}(0)<1 / 2$. Then, for $\phi_{i}, \phi_{-i}>0$ but small enough, we have $\pi^{d}\left(\phi_{-i}\right)<1 / 2 \pi^{m}\left(\phi_{i}\right)$. In this case, the segmentation equilibrium is the unique equilibrium selected by our refinement. To see this, note that in a segmentation equilibrium, sellers on platform $i$ obtain a profit of $1 / 2 \pi^{m}\left(\phi_{i}\right)$ and those on platform $-i$ a profit of $1 / 2 \pi^{m}\left(\phi_{-i}\right)$. A seller active on the platform with the larger fee - for instance, platform $i$ so that $\phi_{i} \geq \phi_{-i}$-has no profitable deviation from this configuration if $1 / 2 \pi^{m}\left(\phi_{i}\right) \geq 1 / 2 \pi^{d}\left(\phi_{-i}\right)$. This implies that for $\pi^{m}\left(\phi_{i}\right) \geq \pi^{d}\left(\phi_{-i}\right)$, segmentation is a Nash equilibrium. In addition, agglomeration is a Nash equilibrium for all fees $\phi_{i}$ such that $D^{d}\left(\phi_{i}\right)>0$. Therefore, multiple Nash equilibria exist in this range. Applying

\footnotetext{
${ }^{51}$ If the demand is unbounded (as, for example, with CES demand), implying that there is no demand intercept, this argument holds true independent of the level of the fee.
} 
coalition-proofness, it is evident from the same arguments as in the previous paragraph that the segmentation equilibrium is eliminated if $\pi^{d}\left(\phi_{-i}\right) \geq 1 / 2 \pi^{m}\left(\phi_{i}\right)$. Thus, if fees are such that $\pi^{d}\left(\phi_{-i}\right) \geq 1 / 2 \pi^{m}\left(\phi_{i}\right)$, the unique equilibrium selected by our refinement is the agglomeration equilibrium on platform $i$ because the segmentation equilibrium is not coalition-proof: ${ }^{52}$

For $\pi^{d}\left(\phi_{-i}\right)<1 / 2 \pi^{m}\left(\phi_{i}\right)$, coalition-proofness does not destroy the segmentation equilibrium. Applying, in addition, profit dominance of sellers, selects the segmentation equilibrium as the unique equilibrium. The reason is that a seller's profit in a segmentation equilibrium is at least $1 / 2 \pi^{m}\left(\phi_{i}\right)$, which is larger than the one in the agglomeration equilibrium, where a seller obtains only $\pi^{d}\left(\phi_{-i}\right)$. Since the condition $\pi^{d}\left(\phi_{-i}\right)<1 / 2 \pi^{m}\left(\phi_{i}\right)$ is stronger than $\pi^{d}\left(\phi_{-i}\right) \leq \pi^{m}\left(\phi_{i}\right)$ (i.e., the condition for a segmentation equilibrium to exist), the segmentation equilibrium is selected by our refinement, whenever the condition holds.

Given our refinement, the equilibrium in the second stage is summarized as follows: Suppose that $\phi_{i} \geq \phi_{-i}$. If $\pi^{d}(0) / \pi^{m}(0) \geq 1 / 2$, agglomeration equilibrium on platform $-i$ occurs. If, by contrast, $\pi^{d}(0) / \pi^{m}(0)<1 / 2$, the segmentation equilibrium is played for $1 / 2 \pi^{m}\left(\phi_{i}\right)>\pi^{d}\left(\phi_{-i}\right)$ and agglomeration on platform $-i$ occurs for $1 / 2 \pi^{m}\left(\phi_{i}\right) \leq \pi^{d}\left(\phi_{-i}\right)$.

We turn to the first stage. Following the same arguments as in the proof of Proposition 1 , it is evident that for $\pi^{d}(0) / \pi^{m}(0) \geq 1 / 2$, the unique equilibrium implies $\left(\phi_{A}^{\star}, \phi_{B}^{\star}\right)=$ $(0,0)$, as sellers will coordinate on the platform with the lower per-transaction fee. This establishes the first part of the proposition.

For $\pi^{d}(0) / \pi^{m}(0)<1 / 2$, we establish next the constraints under which a pure-strategy segmentation equilibrium exists. The highest platform profits that can be obtained in a segmentation equilibrium is reached with fees $\phi_{A}=\phi_{B}=\phi^{m}$. Then, platform $i$ can induce agglomeration only by setting a fee $\phi_{i}$ such that $\pi^{d}\left(\phi_{i}\right) \geq \pi^{m}\left(\phi^{m}\right) / 2$. Denoting the largest such fee by $\phi^{\prime}$ (as in the proposition), such a deviation to $\phi_{i}=\phi^{\prime}$ is not profitable if $\phi^{m} D^{m}\left(\phi^{m}\right) / 2 \geq 2 \phi^{\prime} D^{d}\left(\phi^{\prime}\right)$. This establishes the second part of the proposition, which reports equilibrium transaction fees $\left(\phi_{A}^{\star}, \phi_{B}^{\star}\right)=\left(\phi^{m}, \phi^{m}\right)$.

Finally, by the same arguments as in Section 3, there is no pure-strategy equilibrium in the range such that $\pi^{d}(0) / \pi^{m}(0)<1 / 2$ and $\phi^{m} D^{m}\left(\phi^{m}\right)<4 \phi^{\prime} D^{d}\left(\phi^{\prime}\right)$. In this case, the mixed-strategy equilibrium can be obtained in a similar way as in the proofs of Propositions 3 and 4. In particular, there will again be two regions, one in which mixing occurs on a convex set and the other in which mixing occurs on a non-convex set. Let us characterize the mixed-strategy equilibrium in each of those two regions.

In the region in which mixing occurs on a convex set, we denote the upper and the

\footnotetext{
${ }^{52}$ Note that because $\partial \pi^{m}\left(\phi_{i}\right) / \partial \phi_{i} \leq \partial \pi^{d}\left(\phi_{i}\right) / \partial \phi_{i} \leq 0$, we can have $\pi^{d}(0) / \pi^{m}(0)<1 / 2$ but $\pi^{d}\left(\phi_{-i}\right) \geq$ $1 / 2 \pi^{m}\left(\phi_{i}\right)$ if fees are sufficiently high. Then, a segmentation equilibrium is played if fees are close to zero but an agglomeration one for high fees.
} 
lower bound of the range by $\bar{\phi}$ and $\phi$, respectively. A platform must be indifferent between setting $\bar{\phi}$ and $\underline{\phi}$, which leads to

$$
2 D^{d}(\underline{\phi}) \underline{\phi}=\frac{D^{m}(\bar{\phi}) \bar{\phi}}{2}
$$

In addition, following the same steps as in the proof of Proposition 3, there exists a fee, denoted by $\tilde{\phi}$, in the interior of the randomization domain, which induces segmentation with probability (almost) 1. At this fee, sellers are indifferent between agglomeration and segmentation if one platform charges $\tilde{\phi}$ and the other $\underline{\phi}$, which yields

$$
\pi^{d}(\underline{\phi})=\frac{\pi^{m}(\tilde{\phi})}{2}
$$

The same holds if one platform charges $\tilde{\phi}$ and the other $\bar{\phi}$, which yields

$$
\frac{\pi^{m}(\bar{\phi})}{2}=\pi^{d}(\tilde{\phi})
$$

The three equations 17, , 18), and $(19)$ determine the three fees $\tilde{\phi}, \bar{\phi}$, and $\underline{\phi}$ and, thus, the mixing range. By our assumption that $\pi^{m}$ falls to a larger extent than $\pi^{d}$ with an increase in the per-transaction fee and that the same relation holds true for $D^{m}$ and $D^{d}$, the three fees $\tilde{\phi}, \bar{\phi}$, and $\underline{\phi}$ are uniquely determined.

The best-response function $\phi_{i}\left(\phi_{j}\right)$ is implicitly defined by

$$
\frac{\pi^{m}\left(\phi_{i}\right)}{2}=\pi^{d}\left(\phi_{j}\right) \text { for } \phi_{j}=[\underline{\phi}, \tilde{\phi}]
$$

and

$$
\pi^{d}\left(\phi_{i}\right)=\frac{\pi^{m}\left(\phi_{j}\right)}{2} \text { for } \phi_{j}=(\tilde{\phi}, \bar{\phi}] .
$$

Using these best responses and determining expected profits, we derive the mixing probabilities. We obtain that, in equilibrium,

$$
G(\phi)=\frac{\xi(\phi) D^{m}(\xi(\phi))-\tilde{\phi} D^{m}(\tilde{\phi})}{\xi(\phi) D^{m}(\xi(\phi))} \quad \text { if } \phi=[\underline{\phi}, \tilde{\phi}]
$$

with $\xi(\phi) \equiv\left(\pi^{m}\right)^{-1}\left(2 \pi^{d}(\phi)\right)$, and

$$
G(\phi)=\frac{4 \psi(\phi) D^{d}(\psi(\phi))-\tilde{\phi} D^{m}(\tilde{\phi})}{4 \psi(\phi) D^{d}(\psi(\phi))-\psi(\phi) D^{m}(\psi(\phi))} \quad \text { if } \phi=(\tilde{\phi}, \bar{\phi}]
$$

with $\psi(\phi) \equiv\left(\pi^{d}\right)^{-1}\left(\pi^{m}(\phi) / 2\right)$. The mixing probabilities given by 20) and 21), together with the equations determining $\tilde{\phi}, \bar{\phi}$, and $\underline{\phi}$ characterize the mixed-strategy equilibrium, 
which exists if $\bar{\phi} \leq \phi^{m}$.

To see that $G(\underline{\phi})=0$, note that, from $(18)$, we can write $\xi(\phi)=\tilde{\phi}$. Inserting this into (20) yields $G(\underline{\phi})=0$. Similarly, from $(19)$, we can deduce that $\psi(\bar{\phi})=\tilde{\phi}$. Inserting this into 21 yields $G(\bar{\phi})=1$. To show that there is a mass point at $\phi=\tilde{\phi}$, we can use 20 and (21) to get

$$
\lim _{\phi \nearrow \tilde{\phi}} G(\phi)=\frac{\bar{\phi} D^{m}(\bar{\phi})-\tilde{\phi} D^{m}(\tilde{\phi})}{\bar{\phi} D^{m}(\bar{\phi})}
$$

and

$$
\lim _{\phi \searrow \tilde{\phi}} G(\phi)=\frac{4 \underline{\phi} D^{d}(\underline{\phi})-\tilde{\phi} D^{m}(\tilde{\phi})}{4 \underline{\phi} D^{d}(\underline{\phi})-\underline{\phi} D^{m}(\underline{\phi})}
$$

Using (17), which implies that $\bar{\phi} D^{m}(\bar{\phi})=4 \phi D^{d}(\underline{\phi})$, it is evident that the numerator of the right-hand side of the previous two equations is the same. Comparing the denominators, we obtain $\bar{\phi} D^{m}(\bar{\phi})=4 \underline{\phi} D^{d}(\underline{\phi})>4 \underline{\phi} D^{d}(\underline{\phi})-\tilde{\phi} D^{m}(\tilde{\phi})$. Therefore, the denominator of $\lim _{\phi \nearrow \tilde{\phi}} G(\phi)$ is larger than the one $\lim _{\phi \searrow \tilde{\phi}} G(\phi)$, which yields $\lim _{\phi \nearrow \tilde{\phi}} G(\phi)<\lim _{\phi \searrow \tilde{\phi}} G(\phi)$. Hence, there is a mass point at $\phi=\tilde{\phi}$.

If $\bar{\phi}>\phi^{m}$, this equilibrium cannot exist, as a platform will never find it optimal to set a higher per-transaction fee than $\phi^{m}$. In this region, we obtain an equilibrium with a nonconvex randomization domain. Following the proof of Proposition 4 , the lower interval is given by $\left[\phi^{\prime}, \phi^{\prime}\right]$, where $\underline{\phi}^{\prime}$ is implicitly defined by $2 D^{d}\left(\underline{\phi}^{\prime}\right) \underline{\phi}^{\prime}=D^{m}\left(\phi^{m}\right) \phi^{m} / 2$, and $\phi^{\prime}$ is defined as in the proposition. The upper interval is $\left[\phi^{\prime \prime}, \phi^{m}\right]$, where $\phi^{\prime \prime}$ is implicitly defined by $\pi^{d}\left(\underline{\phi}^{\prime}\right)=\pi^{m}\left(\phi^{\prime \prime}\right) / 2.53$

The existence of mass points at $\phi=\phi^{\prime \prime}$ and $\phi=\phi^{m}$ can be shown as above. The mixing probabilities can be derived in the same way as in the case with a convex set. They are given by

$$
\begin{gathered}
\tilde{G}(\phi)=\frac{\xi(\phi) D^{m}(\xi(\phi))-\phi^{\prime \prime} D^{m}\left(\phi^{\prime \prime}\right)}{\xi(\phi) D^{m}(\xi(\phi))} \quad \text { if } \phi=\left[\underline{\phi}^{\prime}, \phi^{\prime}\right), \\
\tilde{G}(\phi)=\frac{4 \psi(\phi) D^{d}(\psi(\phi))-\phi^{\prime \prime} D^{m}\left(\phi^{\prime \prime}\right)}{4 \psi(\phi) D^{d}(\psi(\phi))-\psi(\phi) D^{m}(\psi(\phi))} \quad \text { if } \phi=\left[\phi^{\prime \prime}, \phi^{m}\right),
\end{gathered}
$$

and

$$
\tilde{G}(\phi)=1 \quad \text { if } \phi=\phi^{m}
$$

Proof of Proposition 8. The third and the fourth stage play out in a similar way as in the baseline model. In the fourth stage, buyers make their buying decisions to maximize utility, and in the third stage, sellers set their product prices, conditional on the number

${ }^{53}$ Due to our assumptions on the shape of the profit and demand functions, all boundaries are unique. 
of sellers in their product category on the platform.

In the first part of the proof, we determine the conditions under which $f_{i}=0, \forall i=$ $1, \ldots, N$, is an equilibrium of the full game. Note that, given $f_{i}=0, \forall i=1, \ldots, N$, as long as in the equilibrium of the second stage at least one platform does not carry any trade, then no platform can profitably deviate by increasing its fee in the first stage. The reason is that, if a platform carried a positive volume of trade in the equilibrium with zero fees, then, after the deviation, sellers and buyers active on this platform would form a coalition and move to one of the platforms with a fee of zero. If the deviating platform carries no trade, a higher fee cannot make this platform better off since it will not attract any buyers and sellers.

To determine the condition under which a platform could profitably deviate from $f_{i}=0$, given that all other platforms charge a fee of zero, we distinguish between the cases $M=k N$ and $M \neq k N$. Recall that $k$ is the largest integer such that $M \geq k N$.

First, we analyze the case $M=k N$. We know from above that, given zero fees, a platform only has an incentive to deviate to a strictly positive fee if all platforms carry a positive volume of trade. The latter can only occur if each platform hosts $k=M / N$ sellers. This leads to a profit per seller of $\pi(k) / N \geq 0$. The most profitable deviation by a coalition in the second stage is then that one seller moves to another platform together with all buyers (as those benefit from the additional seller). The seller's profit is then $\pi(k+1)$. It follows that for

$$
\pi(k+1)<\frac{\pi(k)}{N},
$$

the deviation is not profitable for the seller, and an equilibrium exists in which all $N$ platforms carry a positive volume of trade. To the contrary, if the condition is not fulfilled-i.e., $\pi(k+1) \geq \pi(k) / N$ as in condition (1) - no equilibrium candidate in stage 2 with $N$ platforms carrying positive volumes of trade exists. Then, for $f_{i}=0, \forall i=1, \ldots, N$, only a subset of platforms will carry a positive volume of trade, which implies that no platform can profitable deviate from these fees. This proofs the first part of the proposition for $M=k N$.

Second, consider the case $M \neq k N$. We start by demonstrating that there can never be a coalition-proof equilibrium in which sellers in different categories split differently on the platforms. To see this, consider the case in which all sellers and all platforms are active. Buyers are then only indifferent between platforms if each one is on average host to $M / N$ sellers. To achieve this, we can split the mass of categories in $N$ segments, each with a mass $1 / N$. In each segment, a platform has either $k$ or $k+1$ sellers in the respective categories, according to the following two rules. First, in each segment, a number $N(k+1-M / N)$ of platforms is host to $k$ sellers and a number $N(M / N-k)$ is host to $k+1$ sellers. Then, in each segment of categories, all $M$ sellers are active. 
Second, we allocate to each platform $k$ sellers in $N(k+1)-M$ segments and $k+1$ sellers in $M-N(k)$ segments. Then, summing up over the categories, the average number of sellers on each platform is $M / N+54$

However, such a distribution is not coalition-proof. Take one segment of categories and consider all sellers who are active on a platform with $k+1$ sellers in their categories. Take as a coalition one seller in each category within the segment together with all buyers on the seller's platform. This coalition has an incentive to go to a platform with only $k$ sellers. Per category, the deviating seller then obtains a profit (excluding the listing fee) of $2 \pi(k+1) / N$, whereas without the deviation the profit is only $\pi(k+1) / N$. In addition, also the buyers benefit as they now expect more sellers on the platform than before (i.e., the expected number of sellers is $k+1$ instead of $M / N)$. It follows that there exists a profitable deviation from such an asymmetric equilibrium.

The same argument holds if only a subset of sellers is active. Therefore, if listing fees are symmetric on all platforms, the equilibrium in the second stage must be symmetric across all categories.

We now determine for the case $M \neq k N$, under which conditions $f_{i}=0, \forall i=1, \ldots, N$, is an equilibrium in the first stage. Suppose first that only a subset of platforms has a positive volume of trade. In this situation, sellers and buyers in the second stage choose either agglomeration (that is, all buyers and all active sellers are on one platform) or another distribution in which all platforms with positive market share host the same number of sellers in all categories. The selected equilibrium depends on the profits that sellers obtain and the numbers $M$ and $N 55$ In this situation, following the same arguments as in the case $M=k N$, no platform can profitably increase its fee.

Instead, suppose that all platforms carry a positive volume of trade. Proceeding analogously to the case $M=k N$, we obtain inequality (24) also for the case $M \neq k N$.

We now show that if (24) holds, an equilibrium with zero fees will not be selected by profit-dominance in the first stage. Suppose that (24) holds and all platforms charge strictly positive fees. Then, slightly lowering the fee is not profitable for a platform, as sellers and buyers will still play a segmentation equilibrium in the second stage, in which all platforms have a positive market share and are host to $k$ sellers. As a consequence, an equilibrium with strictly positive fees exists. However, as $M \neq k N$, an equilibrium

\footnotetext{
${ }^{54}$ For example, if $M=11$ and $N=4$, we split the categories in 4 segments, each one with mass $1 / 4$. In the first one of these segments, platform 1 is host to 2 sellers in all categories in the segment, whereas platforms 2, 3, and 4, are host to 3 sellers. In the second segment, platform 2 is host to 2 sellers and all others platforms are host to 3 sellers, whereas in the third (fourth) segment, platform 3 (4) is host to 2 sellers and the other platforms are host to 3 sellers. Then, each platform has on average $11 / 4$ and sellers and buyers are indifferent.

${ }^{55}$ Suppose, for example, that there are 10 sellers per category, 3 platforms, and $\pi(10)>0$. Then, in the first equilibrium type, one platform is host to 10 sellers, whereas in the second equilibrium type, two platforms host 5 sellers each.
} 
in which $f_{i}=0, \forall i=1, \ldots, N$ always exists since at least one platform will not carry a positive trade volume. Yet, the latter is profit-dominated. Therefore, the equilibrium with strictly positive fees will be selected in the first stage, whenever the two equilibria co-exist. To sum up the analysis so far, in case $M \geq N$, a pure-strategy equilibrium in the first stage with $f_{i}=0, \forall i=1, \ldots, N$ exists and is selected if and only if condition (1) is satisfied.

From the preceding arguments, it also follows that for $M<N$, the unique equilibrium involves $f_{i}=0, \forall i=1, \ldots, N$, as in any equilibrium in the second stage, only a subset of platforms can carry a positive amount of trade.

In the second part of the proof, we turn to the segmentation equilibrium with positive fees. From above, we know that for an equilibrium with positive fees to exist, all platforms must carry a positive volume of trade. This implies that each platform will have a mass of $1 / N$ buyers. Suppose that in a segmentation equilibrium candidate, each platform hosts $l \in\{1, \ldots, k\}$ sellers. Then, a platform $i$ can charge at most $f_{i}=\pi(l) / N$, leading to a platform profit of $\Pi_{i}=l \pi(l) / N$ and zero profits to sellers. Consider a coalition of one seller on a platform $j \neq i$ together with all buyers (i.e., not only those on platform $j$ but the whole buyer mass of 1 ). If this coalition deviates to platform $i$, all buyers benefit as they now face $l+1$ sellers instead of only $l$. The seller instead benefits only if $\pi(l+1)>\pi(l) / N$. In addition, it is easy to check that this coalition leads to the tightest condition for a segmentation equilibrium to exist in the second stage, as a deviation involving more than one seller (per category) leads to lower seller profits. It follows that segmentation is an equilibrium in the second stage if and only if $\pi(l) / N \geq \pi(l+1)$.

We turn to the first stage and check if a platform has a profitable deviation from the equilibrium candidate $f_{i}=\pi(l) / N$. Consider the deviation in which platform $i$ sets a fee slightly below $\pi(l+1)$. It then attracts $l+1$ sellers and, thereby, also all buyers. Hence, the deviating platform's profit is $(l+1) \pi(l+1)$. Therefore, this deviation is not profitable if $l \pi(l) / N \geq(l+1) \pi(l+1)$. This condition is (weakly) stronger than $\pi(l) / N \geq \pi(l+1)$, which was derived for the candidate segmentation equilibrium to exist in the second stage. Thus, we can focus on the first stage.

By the same logic as in the previous paragraph, a platform can also deviate to any fee slightly below $\pi(m)$, with $l<m \leq M$. The most profitable deviation is therefore to set a fee of $\pi(\hat{m})$, with $\hat{m} \in \arg \max _{l<m \leq M} m \pi(m)$. It follows that if there exists some $l \in\{1, \ldots, k\}$ such that (2) holds, a pure-strategy segmentation equilibrium exists in which platforms charge $f_{i}=\pi(l) / N$. Applying profit-dominance in the first stage, platforms choose $f_{i}=\pi\left(l^{\star}\right) / N$, such that $l^{\star} \in \arg \max _{l \in\{1, \ldots, k\}} l \pi(l)$ subject to condition (2).

In the third part of the proof, we show that if (1) is not satisfied, this does not imply that (2) holds (i.e., there can be a region in which neither condition is satisfied). To 
see this, note that for $\pi(k) / N>\pi(k+1)$, condition (1) is not satisfied. We now turn to condition (2). Suppose first that $l=k$. Then, (2) holds if $k \pi(k) / N \geq \hat{m} \pi(\hat{m})$ or $\pi(k) / N \geq \hat{m} \pi(\hat{m}) / k$. We know that $\hat{m}>k$, which implies that $\hat{m}$ must be at least $k+1$. Inserting $\hat{m}=k+1$ into $\pi(k) / N \geq \hat{m} \pi(\hat{m}) / k$ yields $\pi(k) / N \geq(k+1) \pi(k+1) / k$. It is easy to see that for

$$
\pi(k+1)<\frac{\pi(k)}{N}<\frac{(k+1) \pi(k+1)}{k}
$$

neither (1) nor (2) is satisfied. Since the right-hand side of (2) is at least as high as $(k+1) \pi(k+1)$ (due to the fact that $\hat{m}$ is chosen to maximize $m \pi(m)$ with respect to $m$ ), this also holds if $\hat{m} \neq k+1$.

A similar argument obtains for the case in which $l \neq k$. Rewriting (2), we obtain $\pi(l) / N \geq \hat{m} \pi(\hat{m}) / l$. The left-hand side of the previous inequality is larger than the righthand side of (1). However, $\pi(k) / N>\pi(k+1)$ does not rule out that there exists an $\hat{m}$, such that $\pi(l) / N<\hat{m} \pi(\hat{m}) / l$ holds. In this case, again neither (1) nor (2) is satisfied.

Showing that there is a unique mixed-strategy equilibrium in this case follows from the same arguments as in the proofs of Propositions 3 and 4 . Platform profits in the mixedstrategy equilibrium are strictly positive. This follows because, if $\pi(k+1) \geq \pi(k) / N$ is violated, each platform sets a strictly positive fee even if all other platforms charge a fee of zero. Therefore, setting a fee equal to zero is not part of the mixing domain. As a consequence, the mixed-strategy equilibrium profit-dominates the pure-strategy equilibrium with $f_{i}^{\star}=0, \forall i=1, \ldots, N$, which exists for the case $M \neq k N$. Therefore, the mixed-strategy equilibrium is always selected in the first stage. 


\section{References}

[1] Affeldt, P., L. Filistrucchi, and T.J. Klein (2013): "Upward Pricing Pressure in Two-Sided Markets," Economic Journal, 123, 505-523.

[2] Anderson, S. and R. Renault (1999): "Pricing, Product Diversity, and Search Costs: A Bertrand-Chamberlin-Diamond Model," RAND Journal of Economics, 30, 719735.

[3] Armstrong, M. (2006): "Competition in Two-Sided Markets," RAND Journal of Economics, 37, 668-691.

[4] Augereau, A., S. Greenstein and M. Rysman (2006): "Coordination versus Differentiation in a Standards War: 56K Modems," RAND Journal of Economics, 37, 887-909.

[5] Aumann, R. (1959): Acceptable Points in General Cooperative N-Person Games, in Contributions to the Theory of Games IV, Princeton University Press, Princeton, New Jersey.

[6] Baye, M.R. and J. Morgan (2001): "Information Gatekeepers on the Internet and the Competitiveness of Homogeneous Product Markets," American Economic Review, $91,454-474$.

[7] Baye, M.R. and J. Morgan (2004): "Price Dispersion in the Lab and on the Internet: Theory and Evidence," RAND Journal of Economics, 35, 449-466.

[8] Belleflamme, P. and E. Toulemonde (2009): "Negative Intra-Group Externalities in Two-Sided Markets," International Economic Review, 50, 245-272.

[9] Bernheim, D., B. Peleg and M. Whinston (1987a): "Coalition-Proof Nash Equilibria I. Concepts," Journal of Economic Theory, 42, 1-12.

[10] Bernheim, D., B. Peleg and M. Whinston (1987b): "Coalition-Proof Nash Equilibria II. Applications," Journal of Economic Theory, 42, 13-29.

[11] Bowley, A. L. (1924): The Mathematical Groundwork of Economics. Oxford: Oxford University Press.

[12] Brown, J.R. and A. Goolsbee (2002): "Does the Internet Make Markets More Competitive? Evidence from the Life Insurance Industry," Journal of Political Economy, 110, 481-507.

[13] Bundeskartellamt (2016): "Market Power of Platforms and Networks,"Working Paper B6-113/15. 
[14] Burguet, R., R. Caminal and M. Ellman (2016): "In Google we Trust?," International Journal of Industrial Organization, 39, 44-55.

[15] Caillaud, B. and B. Jullien (2001): “Competing Cybermediaries," European Economic Review, 45, 797-808.

[16] Caillaud, B. and B. Jullien (2003): "Chicken and Egg: Competition Among Intermediation Service Providers," RAND Journal of Economics, 34, 309-328.

[17] Church, J. and N. Gandal (1992): "Network Effects, Software Provision, and Standardization," Journal of Industrial Economics, 40, 85-103.

[18] Dudey, M. (1990): "Competition by Choice: The Effect of Consumer Search on Firm Location Decisions," American Economic Review, 80, 1092-1104.

[19] Dukes, A. and E. Gal-Or (2003): "Negotiations and Exclusivity Contracts for Advertising," Marketing Science, 22, 222-245.

[20] Edgeworth, F. (1925): "The Pure Theory of Monopoly,"in: Papers Relating to Political Economy, Vol. 1. London: MacMillan, 111-142.

[21] Ellison, G. and D. Fudenberg (2003): "Knife-Edge or Plateau: When Do Market Models Tip?," Quarterly Journal of Economics, 118, 1249-1278.

[22] Ellison, G., D. Fudenberg and M. Möbius (2004): "Competing Auctions," Journal of the European Economic Association, 2, 30-66.

[23] Evans, D. and R. Schmalensee (2007): "The Industrial Organization of Markets with Two-Sided Platforms," Competition Policy International, 3, 151-179.

[24] Galeotti, A. and J.L. Moraga-González (2009): "Platform Intermediation in a Market for Differentiated Products," European Economic Review, 53, 417-428.

[25] Gehrig, T. (1998): “Competing Markets," European Economic Review, 42, 277-310.

[26] Gomes, R. (2014): "Optimal Auction Design in Two-Sided Markets," RAND Journal of Economics, 45, 248-272.

[27] Hagiu, A. (2006): "Pricing and Commitment by Two-Sided Platforms," RAND Journal of Economics, 37, 720-737.

[28] Halaburda, H., M. J. Piskorksi and P. Yilidrim (2017): "Competing by Restricting Choice: The Case of Search Platforms," Management Science, in press.

[29] Janssen, M. and J.-L. Moraga-González (2004): "Strategic Pricing, Consumer Search and the Number of Firms," Review of Economic Studies, 71, 1089-1118. 
[30] Jullien, B. (2011): "Competition in Multi-Sided Markets: Divide and Conquer," American Economic Journal: Microeconomics, 3, 186-219.

[31] Kim, B.-C., J.J. Lee and H. Park (2017): "Two-Sided Platform Competition with Multihoming Agents: An Empirical Study on the Daily Deals Market," Information Economics and Policy, in press.

[32] Lee, R. (2013): "Vertical Integration and Exclusivity in Platform and Two-Sided Markets," American Economic Review, 103, 2960-3000.

[33] Maskin, E. and J. Tirole (1988): "A Theory of Dynamic Oligopoly, II: Price Competition, Kinked Demand Curves, and Edgeworth Cycles," Econometrica, 56, 571-599.

[34] Nocke, V., M. Peitz and K. Stahl (2007): "Platform Ownership," Journal of the European Economic Association, 5, 1130-1160.

[35] Rochet, J.-C. and J. Tirole (2003): "Platform Competition in Two-Sided Markets," Journal of the European Economic Association, 1, 990-1029.

[36] Rochet, J.-C. and J. Tirole (2006): "Two-Sided Markets: A Progress Report," RAND Journal of Economics, 37, 645-667.

[37] Ronayne, D. (2015): "Price Comparison Websites," Warwick Economics Research Paper Series 1056.

[38] Salop, S.C. (1979): "Monopolistic Competition with Outside Goods," Bell Journal of Economics, 10, 141-156.

[39] Singh, N. and X. Vives (1984): "Price and Quantity Competition in a Differentiated Duopoly," RAND Journal of Economics, 15, 546-554.

[40] Stahl, K. (1982): "Differentiated Products, Consumer Search, and Locational Oligopoly," Journal of Industrial Economics, 31, 97-103.

[41] Varian, H.R. (1980): "A Model of Sales," American Economic Review, 70, 651-659.

[42] VRMintel (2016): "Airbnb vs. HomeAway: Who is Winning the Race to the Top of the Vacation Rental Industry?,", available at: http://www.vrmintel.com/airbnb-vshomeaway-winning-race-top-vacation-rental-industry/.

[43] Wolinsky, A. (1986): "True Monopolistic Competition as a Result of Imperfect Information," Quarterly Journal of Economics, 101, 493-511. 